\title{
COVERINGS AND FUNDAMENTAL ALGEBRAS FOR PARTIAL DIFFERENTIAL EQUATIONS
}

\author{
SERGEY IGONIN
}

\begin{abstract}
Following I. S. Krasilshchik and A. M. Vinogradov 8, we regard PDEs as infinite-dimensional manifolds with involutive distributions and consider their special morphisms called differential coverings, which include constructions like Lax pairs and Bäcklund transformations. We show that, similarly to usual coverings in topology, at least for some PDEs differential coverings are determined by actions of a sort of fundamental group. This is not a group, but a certain system of Lie algebras, which generalize WahlquistEstabrook algebras. From this we deduce an algebraic necessary condition for two PDEs to be connected by a Bäcklund transformation. We compute these infinite-dimensional Lie algebras for several $\mathrm{KdV}$ type equations and prove non-existence of Bäcklund transformations.

As a by-product, for some class of Lie algebras $\mathfrak{g}$ we prove that any subalgebra of $\mathfrak{g}$ of finite codimension contains an ideal of $\mathfrak{g}$ of finite codimension.
\end{abstract}

\section{Contents}

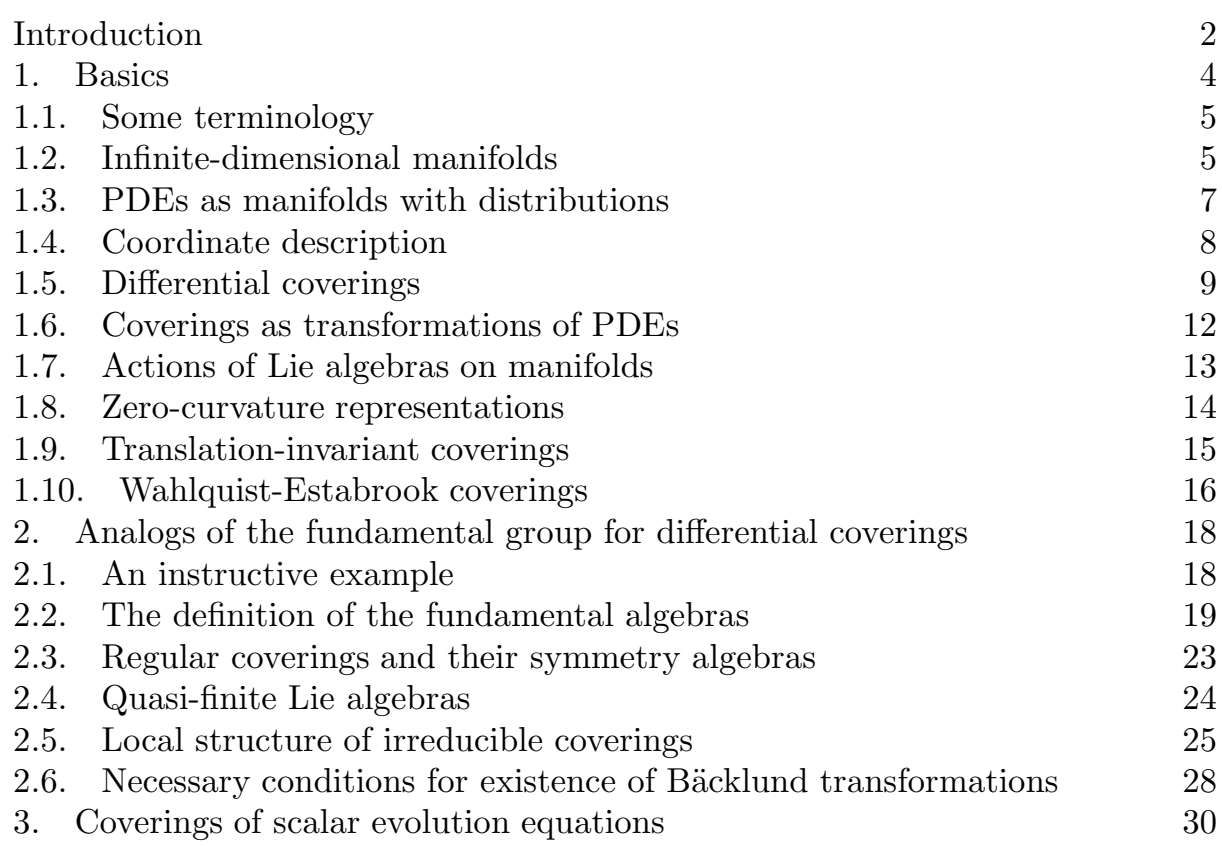

1991 Mathematics Subject Classification. 37K30, 37K35, 35Q53.

Key words and phrases. Coverings of PDEs, Bäcklund transformations, Wahlquist-Estabrook structures, actions of Lie algebras on manifolds, the KdV equation, the Krichever-Novikov equation. 
4. Coverings of the KdV equation 34

4.1. The canonical form of coverings 35

4.2. The fundamental algebras $\quad 37$

5. Coverings of the Krichever-Novikov equation 39

5.1. The canonical form of coverings 40

5.2. The fundamental algebras 42

6. Coverings of the equation $u_{+}=u_{r r x}$. 46

7. Non-existence results for Bäcklund transformations 48

Acknowledgements $\quad 49$

References $\quad 49$

\section{INTRODUCTION}

In this paper we study special correspondences called (differential) coverings between systems of PDEs. Roughly speaking, a covering $\mathcal{E}_{1} \rightarrow \mathcal{E}_{2}$ is a differential mapping from one system $\mathcal{E}_{1}$ to another system $\mathcal{E}_{2}$ such that the preimage of each local solution of $\mathcal{E}_{2}$ is a family of $\mathcal{E}_{1}$ solutions dependent on a finite number $m$ of parameters.

For example, if $v(x, t)$ is a solution of the modified $\mathrm{KdV}$ equation

$$
v_{t}=v_{x x x}-6 v^{2} v_{x}
$$

then the function

$$
u=v_{x}-v^{2}
$$

satisfies the $\mathrm{KdV}$ equation $u_{t}=u_{x x x}+6 u u_{x}$. This is the famous Miura transformation, which determines a covering from the modified $\mathrm{KdV}$ equation to the $\mathrm{KdV}$ equation. For a given local solution $u(x, t)$ of the KdV equation, a one-parameter family of functions $v(x, t)$ is recovered from equations (2) and (11). That is, we have $m=1$ for this covering. In general, systems $\mathcal{E}_{1}$ and $\mathcal{E}_{2}$ may be overdetermined, but must be consistent.

More precisely, following [1, 8, 9, we regard $\mathcal{E}_{1}, \mathcal{E}_{2}$ as submanifolds in infinite jet spaces. The (usually infinite-dimensional) submanifold of infinite jets satisfying a system of PDEs is called the infinite prolongation of the system and possesses a canonical involutive distribution called the Cartan distribution. This distribution is spanned by the total derivative operators (regarded as commuting vector fields on the infinite jet space) with respect to the independent variables. A (differential) covering $\tau: \mathcal{E}_{1} \rightarrow \mathcal{E}_{2}$ is a bundle of finite $\operatorname{rank}^{1} m$ such that the differential $\tau_{*}$ maps the Cartan tangent subspaces of $\mathcal{E}_{1}$ isomorphically onto the ones of $\mathcal{E}_{2}$. Note that even local classification of coverings is highly nontrivial due to different possible configurations of the distributions.

It was shown in [8] that all kinds of Lax pairs, zero-curvature representations, Wahlquist-Estabrook prolongation structures, and Bäcklund transformations in soliton theory are special types of coverings. In particular, a Bäcklund transformation between two systems $\mathcal{E}_{1}$ and $\mathcal{E}_{2}$ is given by another system $\mathcal{E}_{3}$ and a pair of coverings $\mathcal{E}_{1} \leftarrow \mathcal{E}_{3} \rightarrow \mathcal{E}_{2}$.

\footnotetext{
${ }^{1}$ One can consider also coverings of infinite rank 1 [ 8, but we study only the case of finite rank
} 
The name 'coverings' for such bundles is used because they include usual topological coverings of finite-dimensional manifolds, see Example 3 below.

Recall that for a finite-dimensional manifold $M$ its topological coverings are in one-to-one correspondence with actions of the fundamental group $\pi_{1}(M)$ on (discrete) sets. The main result of this paper is that at least for some PDEs $\mathcal{E}$ differential coverings are also determined by actions of a sort of fundamental group. However, this is not a group, but a certain system of Lie algebras that we call the fundamental algebras of $\mathcal{E}$. They are arranged in a sequence of epimorphisms

$$
\cdots \rightarrow \mathfrak{f}_{k+1} \rightarrow \mathfrak{f}_{k} \rightarrow \cdots \rightarrow \mathfrak{f}_{1} \rightarrow \mathfrak{f}_{0}
$$

Differential coverings of rank $m$ are determined by actions of these Lie algebras on $m$-dimensional manifolds $W$, that is, homomorphisms from $\mathfrak{f}_{k}$ to the algebra $D(W)$ of vector fields on $W$. Two coverings are isomorphic if and only if the corresponding actions are isomorphic.

More precisely, the following facts hold:

- for each action $\rho: \mathfrak{f}_{k} \rightarrow D(W)$ we introduce an involutive distribution on the manifold $\mathcal{E} \times W$ such that the trivial bundle $\mathcal{E} \times W \rightarrow \mathcal{E}$ becomes a covering denoted $\tau(\rho)$ (here $\mathcal{E}$ is endowed with the fixed Cartan distribution),

- for any covering $\tau: \tilde{\mathcal{E}} \rightarrow \mathcal{E}$ we define an action $\rho(\tau): \mathfrak{f}_{k} \rightarrow D(\tilde{\mathcal{E}})$ for some $k$ such that $\tau_{*} \rho(\tau)=0$,

- for an action $\rho_{0}: \mathfrak{f}_{k} \rightarrow D(W)$ and the covering $\tau=\tau\left(\rho_{0}\right)$, the action $\rho(\tau)$ is equal to the composition of the natural embedding $D(W) \subset D(\mathcal{E} \times W)$ with the action $\rho_{0}$,

- a morphism of coverings $\tau_{1}$ and $\tau_{2}$ of $\mathcal{E}$ induces a morphism of the actions $\rho\left(\tau_{1}\right)$ and $\rho\left(\tau_{2}\right)$,

- a covering $\tilde{\mathcal{E}} \rightarrow \mathcal{E}$ on a neighborhood of each point of $\tilde{\mathcal{E}}$ is isomorphic to the covering $\tau(\rho)$ for some action $\rho$ of $\mathfrak{f}_{k}$ and some $k$.

The algebra $\mathfrak{f}_{0}$ is equal to the Wahlquist-Estabrook prolongation algebra of $\mathcal{E}[\underline{8}$ 20. 22. To obtain algebras $\mathfrak{f}_{k}$ for $k \geq 1$, we replace the Wahlquist-Estabrook ansatz by jets of arbitrary order and find a canonical form of coverings with respect to the local gauge equivalence.

Note that some similarity between Wahlquist-Estabrook algebras and the topological fundamental group was noticed in [9]. However, before the present paper this idea was not developed and did not lead to any applications.

We prove that all finite-dimensional quotients of the fundamental algebras are coordinate-independent invariants of the system of PDEs. Namely, recall that quotients of the topological fundamental group $\pi_{1}(M)$ occur as automorphism groups of regular topological coverings of $M$. Similarly, finite-dimensional quotients of the fundamental algebras occur as Lie algebras of infinitesimal automorphisms of certain coverings of $\mathcal{E}$.

We conjecture that the fundamental algebras themselves are also coordinateindependent invariants and hope to prove this elsewhere using the homological techniques of [5, 12, 21].

We formulate some conditions for a system of PDEs to possess fundamental algebras. We check these conditions and compute algebras (3) for three PDEs: the $\mathrm{KdV}$ equation, the nonsingular Krichever-Novikov equation, and the linear equation

$$
u_{t}=u_{x x x} .
$$


In all three cases each $\mathfrak{f}_{k}$ is obtained from a single Lie algebra $\mathfrak{K}$ applying several times the operation of one-dimensional central extension.

For the $\mathrm{KdV}$ equation we have $\mathfrak{K}=\mathfrak{s l}_{2}(\mathbb{C}) \otimes_{\mathbb{C}} \mathbb{C}[\lambda]$.

For the nonsingular Krichever-Novikov equation the algebra $\mathfrak{K}$ is isomorphic to a certain subalgebra of the tensor product of $\mathfrak{s l}_{2}(\mathbb{C})$ with the algebra of regular functions on an affine elliptic curve. Note that in this case $\mathfrak{f}_{0}=0$, that is, the Wahlquist-Estabrook ansatz gives no nontrivial coverings.

For equation (4) the algebra $\mathfrak{K}$ possesses a filtration by solvable ideals

$$
\mathfrak{K}_{0} \subset \mathfrak{K}_{1} \subset \cdots \subset \mathfrak{K}_{k} \subset \cdots \subset \mathfrak{K}
$$

such that the quotient $\mathfrak{K} / \cup_{k=0}^{\infty} \mathfrak{K}_{k}$ is solvable as well.

The described methods to compute fundamental algebras can be applied to other evolution equations as well.

In order to develop this theory, we obtain the following results on Lie algebras, which may be of independent interest. A Lie algebra $\mathfrak{g}$ is said to be quasi-finite if any subalgebra of $\mathfrak{g}$ of finite codimension contains an ideal of $\mathfrak{g}$ of finite codimension. We prove that

- a central extension of a quasi-finite algebra is quasi-finite,

- for a finite-dimensional semisimple Lie algebra $\mathfrak{g}$ and a commutative associative algebra $\mathcal{A}$ the tensor product $\mathfrak{g} \otimes \mathcal{A}$ regarded as a Lie algebra is quasi-finite,

- the algebra $\mathfrak{K}$ of the nonsingular Krichever-Novikov equation is quasi-finite.

Recall that for a connected topological covering $\tilde{M} \rightarrow M$ one has $\pi_{1}(\tilde{M}) \subset$ $\pi_{1}(M)$. It turns out that some analog of this property is also valid for differential coverings, see Theorems 12 and 13 .

We obtain also a necessary condition for two systems of PDEs possessing fundamental algebras to be connected by a Bäcklund transformation: their fundamental algebras have to be similar in a certain sense, see Theorem [14 As an example of using this necessary condition, we prove that equation (4) is not connected by any Bäcklund transformation neither with the KdV equation nor with the nonsingular Krichever-Novikov equation. Note that this is apparently the first rigorous non-existence result for Bäcklund transformations.

In this paper we consider only complex-analytic PDEs. Generalization of this theory to smooth PDEs is possible, but is a little more technical, since the analogs of Proposition 3 and Theorem 7 for smooth manifolds do not hold. However, practically all results will remain valid in the smooth case if one excludes from considered manifolds a thin subset of degenerate points. Detailed exposition for smooth PDEs will be done elsewhere.

\section{BASICS}

In this section we review some notions of PDE geometry, actions of Lie algebras on manifolds and prove auxiliary lemmas needed for further theory.

In Subsections 1.2 1.6] we mainly follow [1, 8, 10. However, there are certain modifications because of the fact that we deal with complex-analytic manifolds, while in [1, 8, 10, only smooth manifolds are considered. In particular, we have to use sheaves instead of globally defined functions. Besides, the notions of subequations and irreducible equations are new.

Most of the notions of Subsection 1.7 are studied in more detail in 4 . 
In order to be more readable, all concepts of PDE geometry are introduced in two ways: invariant and coordinate.

1.1. Some terminology. In this paper all manifolds, functions, vector fields, and mappings are supposed to be complex-analytic.

For a manifold $M$ we denote by $D(M)$ the Lie algebra of vector fields on $M$. For a function $f$ on $M$ and a point $a \in M$, the differential of $f$ at $a$ is denoted by $d_{a} f$.

The differential of a mapping $\varphi: M_{1} \rightarrow M_{2}$ of manifolds is denoted by $\varphi_{*}$.

$\mathbb{Z}_{+}$is the set of nonnegative integers.

For subspaces $V_{1}, \ldots, V_{k}$ of a linear space, the space $\left\langle V_{1}, \ldots, V_{k}\right\rangle$ is the linear span of $V_{1}, \ldots, V_{k}$.

In this paper a surjective submersion is called a bundle. To emphasize its properties that in Section 1.2 will be extended to infinite-dimensional manifolds, we give the following definition.

Definition 1. A mapping $\varphi: M_{1} \rightarrow M_{2}$ of manifolds is called a bundle if

- the mapping $\varphi$ is surjective,

- for any point $a \in M_{1}$ there is a neighborhood $a \in U \subset M_{1}$ and a manifold $W$ such that $\varphi(U)$ is open in $M_{2}$ and one has the commutative diagram

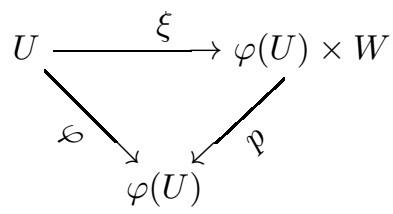

where $\xi$ is a complex-analytic diffeomorphism and $p$ is the projection to the first factor.

In this case the preimages $\varphi^{-1}(b)$ of points $b \in M_{2}$ are submanifolds in $M_{1}$ and are called the fibres of $\varphi$. They are not necessarily isomorphic to each other, but have the same dimension called the rank of $\varphi$.

For a bundle $\varphi: M_{1} \rightarrow M_{2}$, a vector field $V \in D\left(M_{1}\right)$ is said to be $\varphi$-vertical if $\varphi_{*}(V)=0$.

In what follows we say that a certain property holds locally if it holds on a neighborhood of each point of the manifold under consideration.

1.2. Infinite-dimensional manifolds. We want to extend the category of finitedimensional manifolds in order to include certain type of infinite-dimensional manifolds that occur in PDE geometry.

Definition 2. Define a category INF as follows.

- First, an elementary object of INF is an infinite chain of bundles

$$
\stackrel{\varphi_{i+2, i+1}}{\longrightarrow} M^{i+1} \stackrel{\varphi_{i+1, i}}{\longrightarrow} M^{i} \stackrel{\varphi_{i, i-1}}{\longrightarrow} \ldots \stackrel{\varphi_{1,0}}{\longrightarrow} M^{0},
$$

where $M^{i}$ are finite-dimensional manifolds.

Two elementary objects

$$
\left\{M_{1}^{i}, \varphi_{i+1, i}^{1}\right\}, \quad\left\{M_{2}^{i}, \varphi_{i+1, i}^{2}\right\}
$$

such that

$$
\exists p, q \in \mathbb{Z} \quad M_{1}^{i+q}=M_{2}^{i}, \varphi_{i+q+1, i+q}^{1}=\varphi_{i+1, i}^{2} \quad \forall i \geq p
$$

are regarded to be identical. 
Denote by $\mathcal{M}$ elementary object (5). A point of $\mathcal{M}$ is a sequence

$$
\left(a_{0}, a_{1}, \ldots, a_{i}, \ldots\right), \quad a_{i} \in M^{i}, \quad \varphi_{i+1, i}\left(a_{i+1}\right)=a_{i} \quad \forall i \geq 0 .
$$

Let us introduce a topology on the set $|\mathcal{M}|$ of points of $\mathcal{M}$. Let $U$ be an open subset of some $M^{p}$. Denote by $U_{i}, i \geq p$, the preimage of $U$ in $M^{p+i}$ under bundles (5). The subset of points (6) such that $a_{i} \in U_{i}$ for all $i \geq p$ is called the elementary open subset of $|\mathcal{M}|$ corresponding to $U$ and is denoted by $S(U)$. By definition, elementary open subsets form a base of the topology on $|\mathcal{M}|$.

Let us define the structure sheaf of functions on $|\mathcal{M}|$. Each (complexanalytic) function $f: U \rightarrow \mathbb{C}$ determines the following function on $S(U)$

$$
\left(a_{0}, a_{1}, \ldots, a_{i}, \ldots\right) \mapsto f\left(a_{p}\right) .
$$

Such functions on $S(U)$ are said to be elementary. Now let $Z$ be an open subset of $|\mathcal{M}|$. A function $g: Z \rightarrow \mathbb{C}$ belongs to the structure sheaf if and only if for each point $a \in Z$ there is an elementary open subset $S(U)$ such that $a \in S(U) \subset Z$ and the restriction of $g$ to $S(U)$ is an elementary function.

- If

$$
\mathcal{M}_{1}=\left\{M_{1}^{i}, \varphi_{i+1, i}^{1}\right\}, \quad \mathcal{M}_{2}=\left\{M_{2}^{i}, \varphi_{i+1, i}^{2}\right\}
$$

are two elementary objects of INF then a morphism $\psi: \mathcal{M}_{1} \rightarrow \mathcal{M}_{2}$ is given by $\alpha, k \in \mathbb{Z}$ and a system of maps

$$
\psi_{i}: M_{1}^{i+\alpha} \rightarrow M_{2}^{i}, \quad i \geq k,
$$

satisfying

$$
\forall i \geq k \quad \varphi_{i+1, i}^{2} \circ \psi_{i+1}=\psi_{i} \circ \varphi_{i+\alpha+1, i+\alpha}^{1} .
$$

- Now an object of INF is a topological space with a sheaf of complex-valued functions that is locally isomorphic to an elementary object of INF. A mapping of objects of INF is a morphism if locally it is a morphism of elementary objects.

Remark 1. Although this definition is rather sketchy, it is sufficient for us, because all objects of INF considered in this paper are open subobjects of elementary objects.

Example 1. With each finite-dimensional manifold $M$ we associate the following elementary object of INF

$$
\rightarrow M \rightarrow M \rightarrow \cdots \rightarrow M
$$

where all arrows are the identity mappings. This construction identifies the category of finite-dimensional manifolds with a subcategory of INF.

Let $\mathcal{M}$ be an object of INF. The sheaf of vector fields on $\mathcal{M}$ is defined in the standard way as the sheaf of derivations of the structure sheaf. It is a sheaf of modules over the structure sheaf of algebras.

In particular, if $\mathcal{M}$ is elementary object (5) then a tangent vector at a point (6) of $\mathcal{M}$ is a sequence

$$
\left(v_{0}, v_{1}, \ldots, v_{i}, \ldots\right), \quad v_{i} \in T_{a_{i}} M^{i}, \quad\left(\varphi_{i+1, i}\right)_{*}\left(v_{i+1}\right)=v_{i} \quad \forall i \geq 0 .
$$

The vector space of all tangent vectors at a point $a$ is denoted by $T_{a} \mathcal{M}$. 
A distribution on $\mathcal{M}$ is a locally free subsheaf of submodules of the vector fields sheaf. In other words, a distribution $\mathcal{D}$ of rank $k$ distinguishes for each point $a$ of $\mathcal{M}$ a subspace

$$
\mathcal{D}_{a} \subset T_{a} \mathcal{M}, \quad \operatorname{dim} \mathcal{D}_{a}=k,
$$

such that locally there are vector fields $X_{1}, \ldots, X_{k}$ that span the subspaces $\mathcal{D}_{a}$.

For a finite-dimensional manifold $W$ and an object $\mathcal{M}$ of INF, one defines the object $\mathcal{M} \times W$ of INF as follows. It is sufficient to consider the case when $\mathcal{M}$ is elementary object (5). Then $\mathcal{M} \times W$ is the elementary object

$$
\rightarrow M^{i+1} \times W \stackrel{\varphi_{i+1, i} \times \mathrm{id}}{\longrightarrow} M^{i} \times W \rightarrow \cdots \rightarrow M^{0} \times W .
$$

Now one easily extends Definition [1 of bundles to the case when $M_{1}, M_{2}$ are objects of INF. However, we always assume the fibres $W$ to be finite-dimensional manifolds.

In what follows, when we speak of functions on an object of INF, we always assume that the functions belong to the structure sheaf.

For the sake of simplicity, below objects of INF are also called manifolds, and morphisms of INF are called mappings.

1.3. PDEs as manifolds with distributions. Let $\pi: E \rightarrow M$ be a bundle of finite-dimensional manifolds and

$$
\theta \in E, \quad \pi(\theta)=x \in M .
$$

Consider a local section $f$ of $\pi$ whose graph passes through the point $\theta$. Denote by $[f]_{x}^{k}$ the class of all local sections whose graphs are tangent to the graph of $f$ at $\theta$ with order $\geq k$. The set

$$
J^{k}(\pi)=\left\{[f]_{x}^{k} \mid f \text { is a local section of } \pi, x \in M\right\}
$$

carries a natural structure of a manifold and is called the manifold of $k$-jets of the bundle $\pi$. Moreover, the natural projections

$$
\begin{gathered}
\pi_{k}: J^{k}(\pi) \rightarrow M, \quad[f]_{x}^{k} \mapsto x, \\
\pi_{k, k-1}: J^{k}(\pi) \rightarrow J^{k-1}(\pi), \quad[f]_{x}^{k} \mapsto[f]_{x}^{k-1},
\end{gathered}
$$

are bundles. The infinite sequence of bundles

$$
\cdots \rightarrow J^{k}(\pi) \stackrel{\pi_{k, k-1}}{\longrightarrow} J^{k-1}(\pi) \rightarrow \cdots \rightarrow J^{1}(\pi) \stackrel{\pi_{1,0}}{\longrightarrow} J^{0}(\pi)=E
$$

determines an object of INF that is called the manifold of infinite jets of $\pi$ and is denoted by $J^{\infty}(\pi)$.

For each local section $f$ of $\pi$ we have the local sections

$$
j_{k}(f): M \rightarrow J^{k}(\pi), \quad x \mapsto[f]_{x}^{k},
$$

of the bundles $\pi_{k}, k=0,1, \ldots$ These sections determine the local section

$$
j_{\infty}(f): M \rightarrow J^{\infty}(\pi)
$$

of the natural projection $\pi_{\infty}: J^{\infty}(\pi) \rightarrow M$.

There is a unique distribution $\mathcal{C}$ on $J^{\infty}(\pi)$ such that for any point $x \in M$ and any local section $f$ of $\pi$ over a neighborhood of $x$ we have

$$
\mathcal{C}_{j_{\infty}(f)(x)}=j_{\infty}(f)_{*}\left(T_{x} M\right) .
$$

This distribution is of $\operatorname{rank} \operatorname{dim} M$ and is called the Cartan distribution of $J^{\infty}(\pi)$. 
Consider a system of PDEs of order $k$ imposed on sections of the bundle $\pi$. We assume that it determines a submanifold $\mathcal{E}^{0} \subset J^{k}(\pi)$ of the manifold $J^{k}(\pi)$ such that the mapping $\left.\pi_{k}\right|_{\mathcal{E}^{0}}: \mathcal{E}^{0} \rightarrow M$ is a bundle. Then a local section $f$ of $\pi$ is a solution of the system of PDEs if and only if the graph of $j_{k}(f)$ is contained in $\mathcal{E}^{0}$.

For each $l \in \mathbb{Z}_{+}$the $l$-th prolongation of $\mathcal{E}^{0}$ is the set

$$
\begin{aligned}
\mathcal{E}^{l}=\left\{[f]_{x}^{k+l} \in J^{k+l}(\pi) \mid \text { the graph of } j_{k}(f) \text { is tangent to } \mathcal{E}^{0}\right. \\
\text { with order } \left.\geq l \text { at }[f]_{x}^{k} \in \mathcal{E}^{0}\right\},
\end{aligned}
$$

$l=0,1, \ldots$ Restricting the maps $\pi_{k+l, k+l-1}$ to $\mathcal{E}^{l}$ and preserving the same notation for these restrictions, we obtain the sequence of maps

$$
\cdots \rightarrow \mathcal{E}^{l} \stackrel{\pi_{k+l, k+l-1}}{\longrightarrow} \mathcal{E}^{l-1} \rightarrow \cdots \rightarrow \mathcal{E}^{0} .
$$

Imposing natural conditions of regularity, we assume that all $\mathcal{E}^{l}$ are submanifolds of $J^{k+l}(\pi)$, while mappings (9) are bundles. The obtained object $\mathcal{E}$ of INF is called the infinite prolongation of the initial system of PDEs.

In what follows all considered systems of PDEs are supposed to satisfy these regularity assumptions and, therefore, possess infinite prolongations. Below such object $\mathcal{E}$ of INF is sometimes simply called an equation.

The distribution $\mathcal{C}$ is tangent to $\mathcal{E}$. Its restriction to $\mathcal{E}$ is denoted by $\mathcal{C}_{\mathcal{E}}$ and is called the Cartan distribution of $\mathcal{E}$. It satisfies $\left[\mathcal{C}_{\mathcal{E}}, \mathcal{C}_{\mathcal{E}}\right] \subset \mathcal{C}_{\mathcal{E}}$. Since $\mathcal{E}$ is infinitedimensional, this does not generally imply existence and uniqueness of maximal integral submanifolds.

Definition 3. Let $\mathcal{E}$ be an object of INF and $\mathcal{D}$ be a distribution on it. A subset $\mathcal{E}^{\prime} \subset \mathcal{E}$ is called a subequation of the pair $(\mathcal{E}, \mathcal{D})$ if $\mathcal{E}^{\prime}$ is a submanifold of codimension $l<\infty$ and $\mathcal{D}$ is tangent to $\mathcal{E}^{\prime}$. More precisely, this means the following. We have $\mathcal{E}^{\prime} \neq \emptyset$, and for each point $a \in \mathcal{E}^{\prime}$ there are a neighborhood $a \in U \subset \mathcal{E}$ and functions $f_{1}, \ldots, f_{l}$ on $U$ such that

- $\mathcal{E}^{\prime} \cap U=\left\{q \in U \mid f_{1}(q)=\cdots=f_{l}(q)=0\right\}$,

- for any $b \in U$ the differentials $d_{b} f_{1}, \ldots, d_{b} f_{l} \in T_{b}^{*} \mathcal{E}$ are linearly independent,

- the ideal of functions on $U$ generated by $f_{1}, \ldots, f_{l}$ is preserved by the action of vector fields from $\mathcal{D}$.

In this case $\mathcal{E}^{\prime}$ is also an object of $\mathbf{I N F}$ with the distribution $\left.\mathcal{D}\right|_{\mathcal{E}^{\prime}}$. The number $l$ is called the codimension of the subequation $\mathcal{E}^{\prime}$.

A pair $(\mathcal{E}, \mathcal{D})$ is said to be irreducible if $\mathcal{E}$ is connected as a topological space and there is no subequation $\mathcal{E}^{\prime} \subset \mathcal{E}$ of finite nonzero codimension.

Let $\mathcal{E}$ be the infinite prolongation of a system of PDEs. Then subequations of $\mathcal{E}$ are subequations of the pair $\left(\mathcal{E}, \mathcal{C}_{\mathcal{E}}\right)$, and $\mathcal{E}$ is called irreducible if the pair $\left(\mathcal{E}, \mathcal{C}_{\mathcal{E}}\right)$ is irreducible.

Remark 2. The term 'subequation' is motivated by the fact that a pair $\left(\mathcal{E}, \mathcal{C}_{\mathcal{E}}\right)$, as we agreed above, is sometimes called an equation.

1.4. Coordinate description. Consider a bundle $\pi: E \rightarrow M$. Let $x_{1}, \ldots, x_{n}$ be local coordinates in $M$ and $u^{1}, \ldots, u^{d}$ be local coordinates in fibres of $\pi$. For a symmetric multi-index $\sigma=i_{1} \ldots i_{k}$ set

$$
u_{\sigma}^{j}=\frac{\partial^{k} u^{j}}{\partial x_{i_{1}} \ldots \partial x_{i_{k}}} .
$$


These functions along with $x_{1}, \ldots, x_{n}$ form a system of local coordinates for the infinite-dimensional space $J^{\infty}(\pi)$. The topology on $J^{\infty}(\pi)$ is the following. Choose a finite number $u_{\sigma_{1}}^{j_{1}}, \ldots, u_{\sigma_{r}}^{j_{r}}$ of coordinates (10) and consider the mapping

$$
J^{\infty}(\pi) \rightarrow \mathbb{C}^{n+r}, \quad a \mapsto\left(x_{1}(a), \ldots, x_{n}(a), u_{\sigma_{1}}^{j_{1}}(a), \ldots, u_{\sigma_{r}}^{j_{r}}(a)\right) .
$$

The preimages of open subsets of $\mathbb{C}^{n+r}, r \in \mathbb{Z}_{+}$, under such mappings are by definition open subsets of $J^{\infty}(\pi)$ and form a base of the topology on $J^{\infty}(\pi)$. Admissible functions on open subsets of $J^{\infty}(\pi)$ may depend on $x_{1}, \ldots, x_{n}$ and a finite number of coordinates (10). Below all functions are supposed to be admissible.

The total derivative operators

$$
D_{x_{i}}=\frac{\partial}{\partial x_{i}}+\sum_{\sigma, j} u_{\sigma i}^{j} \frac{\partial}{\partial u_{\sigma}^{j}}, \quad i=1, \ldots, n,
$$

are commuting vector fields on $J^{\infty}(\pi)$ and span the Cartan distribution.

Consider a system of PDEs

$$
F_{\alpha}\left(x_{i}, u^{k}, u_{\sigma}^{j}, \ldots\right)=0, \quad \alpha=1, \ldots, s,
$$

in the bundle $\pi$. The basic idea of the described approach is to treat (12) not as differential equations in $u^{k}$, but as analytic equations in variables (10) and $x_{i}$.

The differential consequences of (12) are

$$
D_{x_{i_{1}}} \ldots D_{x_{i_{r}}}\left(F_{\alpha}\right)=0, \quad i_{k}=1, \ldots, n, \quad \alpha=1, \ldots, s, \quad r=0,1, \ldots
$$

The infinite prolongation $\mathcal{E} \subset J^{\infty}(\pi)$ of system (12) is distinguished by equations (131). The vector fields $D_{x_{i}}$ are tangent to $\mathcal{E}$, and their restrictions to $\mathcal{E}$ will be denoted by the same symbol $D_{x_{i}}$. They span the Cartan distribution $\mathcal{C}_{\mathcal{E}}$ of $\mathcal{E}$.

Example 2. Consider a scalar evolution equation in two independent variables $x, t$

$$
u_{t}=F\left(x, t, u, u_{1}, u_{2}, \ldots, u_{p}\right), \quad u_{k}=\frac{\partial^{k} u}{\partial x^{k}}, \quad u=u_{0} .
$$

Its infinite prolongation has the natural coordinates $x, t, u_{k}, k \geq 0$, since using differential consequences of (14) all $t$-derivatives are expressed in terms of these. The total derivative operators are written in these coordinates as follows

$$
D_{x}=\frac{\partial}{\partial x}+\sum_{j \geq 0} u_{j+1} \frac{\partial}{\partial u_{j}}, \quad D_{t}=\frac{\partial}{\partial t}+\sum_{j \geq 0} D_{x}^{j}(F) \frac{\partial}{\partial u_{j}} .
$$

\subsection{Differential coverings.}

Definition 4. Let $\mathcal{E}$ be an object of INF endowed with a distribution $\mathcal{D}$ such that $[\mathcal{D}, \mathcal{D}] \subset \mathcal{D}$. A (differential) covering of (or over) the pair $(\mathcal{E}, \mathcal{D})$ is given by a bundle of finite rank

$$
\tau: \tilde{\mathcal{E}} \rightarrow \mathcal{E}
$$

and a distribution $\mathcal{D}^{\tau}$ on $\tilde{\mathcal{E}}$ such that

- $\left[\mathcal{D}^{\tau}, \mathcal{D}^{\tau}\right] \subset \mathcal{D}^{\tau}$

- for each $a \in \tilde{\mathcal{E}}$ the differential $\tau_{*}$ maps the space $\left(\mathcal{D}^{\tau}\right)_{a} \subset T_{a} \tilde{\mathcal{E}}$ isomorphically onto the space $\mathcal{D}_{\tau(a)} \subset T_{\tau(a)} \mathcal{E}$. 
An invertible mapping $\varphi: \tilde{\mathcal{E}} \rightarrow \tilde{\mathcal{E}}$ such that $\tau \circ \varphi=\tau$ is called a gauge transformation. The covering given by the same bundle $\tau$ and the new distribution $\varphi_{*}\left(\mathcal{D}^{\tau}\right)$ on $\tilde{\mathcal{E}}$ is said to be (gauge) equivalent to the initial covering.

Similarly, a morphism between two coverings $\tau_{i}: \mathcal{E}_{i} \rightarrow \mathcal{E}, i=1,2$, over the same pair $(\mathcal{E}, \mathcal{D})$ is a mapping $\varphi: \mathcal{E}_{1} \rightarrow \mathcal{E}_{2}$ such that $\tau_{1}=\tau_{2} \circ \varphi$ and $\varphi_{*}\left(\mathcal{D}^{\tau_{1}}\right) \subset \mathcal{D}^{\tau_{2}}$.

A $\tau$-vertical vector field $X \in D(\tilde{\mathcal{E}})$ is called a (gauge) symmetry of $\tau$ if $\left[X, \mathcal{D}^{\tau}\right] \subset$ $\mathcal{D}^{\tau}$. This means that the local flow of $X$ (if it exists) consists of automorphisms of $\tau$. The Lie algebra of symmetries is denoted by $\operatorname{Sym} \tau$.

Covering (15) is said to be irreducible if both pairs $(\mathcal{E}, \mathcal{D})$ and $\left(\tilde{\mathcal{E}}, \mathcal{D}^{\tau}\right)$ are irreducible.

Example 3. Let us show that usual topological coverings are a particular case of this construction. Let $M$ be a finite-dimensional manifold and $\mathcal{D}$ be the whole tangent bundle of $M$. Coverings of rank 0 over $(M, \mathcal{D})$ are just topological coverings $\tau: \tilde{M} \rightarrow M$, where $\operatorname{dim} \tilde{M}=\operatorname{dim} M$ and $\mathcal{D}^{\tau}$ is the whole tangent bundle of $\tilde{M}$.

If the distribution on $\mathcal{E}$ is clearly fixed, we speak of coverings over $\mathcal{E}$ (without mentioning the distribution).

Let now $\mathcal{E}$ be the infinite prolongation of a system of PDEs (12). In this case we fix $\mathcal{D}$ to be the Cartan distribution $\mathcal{C}_{\mathcal{E}}$.

Let us describe a covering (15) in local coordinates. Recall that locally $\mathcal{C}_{\mathcal{E}}$ is spanned by $D_{x_{i}}$. Therefore, locally there is a unique $n$-tuple of vector fields

$$
\tilde{D}_{x_{i}} \in \mathcal{D}^{\tau}, \quad i=1, \ldots, n,
$$

on the manifold $\tilde{\mathcal{E}}$ such that

$$
\begin{gathered}
\tau_{*}\left(\tilde{D}_{x_{i}}\right)=D_{x_{i}}, \\
{\left[\tilde{D}_{x_{i}}, \tilde{D}_{x_{j}}\right]=0, \quad \forall i, j=1, \ldots, n .}
\end{gathered}
$$

Moreover, vector fields (16) span the distribution $\mathcal{D}^{\tau}$.

If $X \in \operatorname{Sym} \tau$ then we have

$$
\left[X, \tilde{D}_{x_{i}}\right]=0, \quad i=1, \ldots, n .
$$

Below in this section we consider equations in two independent variables $x$ and $t$, i.e., $n=2$. Locally the bundle $\tau$ is trivial

$$
\tau: \mathcal{E} \times W \rightarrow \mathcal{E}, \quad \operatorname{dim} W=m<\infty .
$$

Let $w^{1}, \ldots, w^{m}$ be local coordinates in $W$.

From (17) we have

$$
\tilde{D}_{x}=D_{x}+A, \quad \tilde{D}_{t}=D_{t}+B,
$$

where

$$
A=\sum_{j=1}^{m} a^{j} \frac{\partial}{\partial w^{j}}, \quad B=\sum_{j=1}^{m} b^{j} \frac{\partial}{\partial w^{j}}
$$

are $\tau$-vertical vector fields on $\mathcal{E} \times W$. Condition (18) is written as

$$
D_{x} B-D_{t} A+[A, B]=0,
$$

where

$$
D_{x} B=\sum_{j=1}^{m} D_{x}\left(b^{j}\right) \frac{\partial}{\partial w^{j}}, \quad D_{t} A=\sum_{j=1}^{m} D_{t}\left(a^{j}\right) \frac{\partial}{\partial w^{j}} .
$$


A covering equivalent to the one given by $A=B=0$ is called trivial.

The manifold $\mathcal{E} \times W$ is itself isomorphic to the infinite prolongation of the system that consists of equations (12) and the following additional equations

$$
\begin{aligned}
\frac{\partial w^{j}}{\partial x} & =a^{j}\left(x, t, w^{k}, u_{\sigma}^{i}, \ldots\right), \quad j=1, \ldots, m . \\
\frac{\partial w^{j}}{\partial t} & =b^{j}\left(x, t, w^{k}, u_{\sigma}^{i}, \ldots\right),
\end{aligned}
$$

This overdetermined system is consistent modulo (12) if and only if (23) holds on $\mathcal{E}$. The vector fields $D_{x}+A, D_{t}+B$ are the restrictions of the total derivative operators to $\mathcal{E} \times W$. That is, the distribution $\mathcal{D}^{\tau}$ is the Cartan distribution of this system.

Gauge transformations correspond to invertible changes of variables

$$
x \mapsto x, t \mapsto t, u_{\sigma}^{i} \mapsto u_{\sigma}^{i}, \quad w^{j} \mapsto g^{j}\left(x, t, w^{k}, u_{\sigma}^{i}, \ldots\right), \quad j=1, \ldots, m,
$$

in (24). A covering is trivial if and only if it is obtained by such change of variables from the trivial system

$$
\frac{\partial w^{j}}{\partial x}=\frac{\partial w^{j}}{\partial t}=0, \quad j=1, \ldots, m
$$

Therefore, classification of coverings over $\mathcal{E}$ up to local isomorphism is equivalent to classification of consistent modulo (12) systems (24) up to locally invertible changes of variables (25).

Example 4. Consider a covering of rank 1

$$
\frac{\partial w}{\partial x}=a\left(x, t, w, u, u_{1}, \ldots, u_{k}\right), \quad \frac{\partial w}{\partial t}=b\left(x, t, w, u, u_{1}, \ldots, u_{k}\right)
$$

over the infinite prolongation of equation (14). After a gauge transformation

$$
w \mapsto f\left(x, t, w, u, u_{1}, \ldots, u_{r}\right), \quad \frac{\partial f}{\partial w} \neq 0,
$$

system (26) changes to the following system

$$
\begin{gathered}
\frac{\partial w}{\partial x}=\frac{1}{\frac{\partial f}{\partial w}}\left(a\left(x, t, f, u, u_{1}, \ldots, u_{k}\right)-D_{x} f\right), \\
\frac{\partial w}{\partial t}=\frac{1}{\frac{\partial f}{\partial w}}\left(b\left(x, t, f, u, u_{1}, \ldots, u_{k}\right)-D_{t} f\right), \\
\quad f=f\left(x, t, w, u, u_{1}, \ldots, u_{r}\right),
\end{gathered}
$$

which represents an equivalent to (26) covering.

Recall that in the case of two independent variables $x, t$ a conserved current of $\mathcal{E}$ is a pair of functions $(f, g)$ on $\mathcal{E}$ satisfying

$$
D_{t} f=D_{x} g \text {. }
$$

Two conserved currents $\left(f_{1}, g_{1}\right)$ and $\left(f_{2}, g_{2}\right)$ are called equivalent if there is a function $h$ such that

$$
f_{2}-f_{1}=D_{x}(h), \quad g_{2}-g_{1}=D_{t}(h) .
$$

For a conserved current (27) the pair of vector fields

$$
A=f\left(x, t, u_{\sigma}^{i}, \ldots\right) \frac{\partial}{\partial w}, \quad B=g\left(x, t, u_{\sigma}^{i}, \ldots\right) \frac{\partial}{\partial w}
$$


satisfies (23) and determines a covering of rank 1.

Equivalent conserved currents (28) determine equivalent coverings. Indeed, the corresponding gauge transformation is $w \mapsto w+h$.

1.6. Coverings as transformations of PDEs. Consider two systems of PDEs

$$
\begin{aligned}
& F_{\alpha}\left(x, t, u^{1}, \ldots, u^{d_{1}}, \frac{\partial^{p+q} u^{j}}{\partial x^{p} \partial t^{q}}, \ldots\right)=0, \quad \alpha=1, \ldots, s_{1}, \\
& G_{\alpha}\left(x, t, v^{1}, \ldots, v^{d_{2}}, \frac{\partial^{p+q} v^{j}}{\partial x^{p} \partial t^{q}}, \ldots\right)=0, \quad \alpha=1, \ldots, s_{2},
\end{aligned}
$$

and a mapping

$$
u^{j}=\varphi^{j}\left(x, t, v^{1}, \ldots, v^{d_{2}}, \frac{\partial^{p+q} v^{l}}{\partial x^{p} \partial t^{q}}, \ldots\right), \quad j=1, \ldots, d_{1},
$$

such that the following conditions hold.

(1) For each local solution $v^{1}(x, t), \ldots, v^{d_{2}}(x, t)$ of system (30) functions 31) constitute a local solution of (29).

(2) For each local solution $u^{1}(x, t), \ldots, u^{d_{1}}(x, t)$ of (29) the system that consists of equations (30) and (31) is consistent and possesses locally a general solution

$$
v^{1}\left(x, t, c_{1}, \ldots, c_{m}\right), \ldots, v^{d_{2}}\left(x, t, c_{1}, \ldots, c_{m}\right)
$$

dependent on a finite number of complex parameters $c_{1}, \ldots, c_{m}$.

Example 5. Miura transformation (2) satisfies these conditions with $m=1$.

Consider the following trivial bundles

$$
\begin{aligned}
& \pi: \mathbb{C}^{d_{1}+2} \rightarrow \mathbb{C}^{2},\left(x, t, u^{1}, \ldots, u^{d_{1}}\right) \mapsto(x, t), \\
& \tilde{\pi}: \mathbb{C}^{d_{2}+2} \rightarrow \mathbb{C}^{2}, \quad\left(x, t, v^{1}, \ldots, v^{d_{2}}\right) \mapsto(x, t),
\end{aligned}
$$

and their infinite jet spaces $J^{\infty}(\pi)$ and $J^{\infty}(\tilde{\pi})$.

Denote by $D_{x}, D_{t}$ and $\tilde{D}_{x}, \tilde{D}_{t}$ the total derivative operators on $J^{\infty}(\pi)$ and $J^{\infty}(\tilde{\pi})$ respectively. One has

$$
\frac{\partial^{p+q} u^{j}}{\partial x^{p} \partial t^{q}}=D_{x}^{p} D_{t}^{q}\left(u^{j}\right), \quad \frac{\partial^{p+q} v^{j}}{\partial x^{p} \partial t^{q}}=\tilde{D}_{x}^{p} \tilde{D}_{t}^{q}\left(v^{j}\right) .
$$

Formulas (31) suggest to consider the mapping

$$
\tau: J^{\infty}(\tilde{\pi}) \rightarrow J^{\infty}(\pi)
$$

defined as follows

$$
\tau^{*}(x)=x, \quad \tau^{*}(t)=t, \quad \tau^{*}\left(u^{j}\right)=\varphi^{j}, \quad \tau^{*}\left(\frac{\partial^{p+q} u^{j}}{\partial x^{p} \partial t^{q}}\right)=\tilde{D}_{x}^{p} \tilde{D}_{t}^{q}\left(\varphi^{j}\right) .
$$

Then we obtain

$$
\tau_{*}\left(\tilde{D}_{x}\right)=D_{x}, \quad \tau_{*}\left(\tilde{D}_{t}\right)=D_{t} .
$$

Let $\mathcal{E} \subset J^{\infty}(\pi)$ and $\tilde{\mathcal{E}} \subset J^{\infty}(\tilde{\pi})$ be the infinite prolongations of systems (29) and (30) respectively. Conditions 1 and 2 above need rigorous analytical explanation, which we do not consider. Instead, following [1, 8, we say that Conditions 1 and 2 are by definition equivalent to the fact that $\tau(\tilde{\mathcal{E}})=\mathcal{E}$ and the mapping

$$
\left.\tau\right|_{\tilde{\mathcal{E}}}: \tilde{\mathcal{E}} \rightarrow \mathcal{E}
$$

is a bundle of rank $m$. Then from (34) we obtain that (35) is a covering. 
According to construction (24), every covering of a system of PDEs is locally isomorphic to a covering of this form.

1.7. Actions of Lie algebras on manifolds. Let $\mathfrak{g}$ be a Lie algebra over $\mathbb{C}$. Recall that an action of the Lie algebra $\mathfrak{g}$ on a complex manifold $W$ is a homomorphism $\mathfrak{g} \rightarrow D(W)$. For $a \in W$ let $\mathrm{ev}_{a}: D(W) \rightarrow T_{a} W$ be the evaluation mapping. For an action $\rho: \mathfrak{g} \rightarrow D(W)$ the subalgebra $\left\{v \in \mathfrak{g} \mid \mathrm{ev}_{a} \rho(v)=0\right\}$ is called the isotropy subalgebra of the point $a$.

An action $\rho$ is said to be transitive if the mapping $\operatorname{ev}_{a} \rho: \mathfrak{g} \rightarrow T_{a} W$ is surjective for each $a \in W$. An action $\rho$ is called free if $\operatorname{ker} \operatorname{ev}_{a} \rho=0$ for any $a \in W$.

A bundle $W \rightarrow W^{\prime}$ is called the quotient map with respect to an action $\rho: \mathfrak{g} \rightarrow$ $D(W)$ if all vector fields from $\rho(\mathfrak{g})$ are tangent to the fibres and the induced action on each fibre is transitive.

A morphism from one action $\rho_{1}: \mathfrak{g} \rightarrow D\left(W_{1}\right)$ to another action $\rho_{2}: \mathfrak{g} \rightarrow D\left(W_{2}\right)$ is a mapping $\psi: W_{1} \rightarrow W_{2}$ such that

$$
\forall a \in W_{1} \quad \forall v \in \mathfrak{g} \quad \psi_{*}\left(\operatorname{ev}_{a} \rho_{1}(v)\right)=\operatorname{ev}_{\psi(a)} \rho_{2}(v) .
$$

The following statement is obvious.

Lemma 1. Let $\psi: W_{1} \rightarrow W_{2}$ be a morphism of transitive actions $\rho_{i}: \mathfrak{g} \rightarrow D\left(W_{i}\right)$, $i=1,2$. Then $\psi\left(W_{1}\right)$ is open in $W_{2}$.

Let $G$ be a connected complex Lie group associated with a finite-dimensional Lie algebra $\mathfrak{g}$. For $g \in G$ set

$$
L_{g}: G \rightarrow G, a \mapsto g a, \quad R_{g}: G \rightarrow G, a \mapsto a g .
$$

A vector field $X \in D(G)$ is said to be right invariant if

$$
\forall g \in G \quad\left(R_{g}\right)_{*}(X)=X,
$$

and $X$ is said to be left invariant if

$$
\forall g \in G \quad\left(L_{g}\right)_{*}(X)=X .
$$

Denote by $D_{\text {li }}, D_{\text {ri }} \subset D(G)$ the subalgebras of left invariant and right invariant vector fields respectively. It is well known that

$$
D_{\text {li }} \cong D_{\text {ri }} \cong \mathfrak{g} \text {. }
$$

and the actions of the algebras $D_{\mathrm{li}}, D_{\text {ri }}$ on $G$ are free and transitive.

By isomorphisms (39), we have the free transitive action $\sigma: \mathfrak{g} \rightarrow D(G)$ of $\mathfrak{g}$ on $G$ by right invariant vector fields. Let $H \subset G$ be a connected Lie subgroup and $\mathfrak{h} \subset \mathfrak{g}$ be the corresponding Lie subalgebra. Consider the quotient space $G / H$ with the canonical projection $p: G \rightarrow G / H$.

Due to equation (37), all right invariant vector fields are mapped by $p_{*}$ to welldefined vector fields on $G / H$. Consider the arising transitive action

$$
\sigma_{\mathfrak{h}}=p_{*} \circ \sigma: \mathfrak{g} \rightarrow D(G / H)
$$

of $\mathfrak{g}$ on $G / H$. The following lemma is easy to prove.

Lemma 2. Let $U$ be a connected open subset of $G / H$. Let $X \in D(U)$ commute with all vector fields from $\sigma_{\mathfrak{h}}(\mathfrak{g})$. Then there is $V \in D_{\mathrm{li}}$ such that $X=p_{*}(V)$.

And vice versa, if $V \in D_{\mathrm{li}}$ is projectable to $G / H$ then $p_{*}(V)$ commutes with all vector fields from $\sigma_{\mathfrak{h}}(\mathfrak{g})$. An element $V \in D_{\mathrm{li}} \cong \mathfrak{g}$ is projectable to $G / H$ if and only if $[V, \mathfrak{h}] \subset \mathfrak{h}$. 
In particular, if $U$ is a connected open subset of $G$ then the algebra

$$
\{V \in D(U) \mid[V, \sigma(\mathfrak{g})]=0\}
$$

coincides with $D_{\mathrm{li}} \cong \mathfrak{g}$.

Lemma 3. Let $W$ be a connected finite-dimensional manifold. Suppose that an action $\rho: \mathfrak{g} \rightarrow D(W)$ is free and transitive. Then the Lie algebra

$$
\{V \in D(W) \mid[V, \rho(\mathfrak{g})]=0\}
$$

is isomorphic to $\mathfrak{g}$ and acts on $W$ freely and transitively as well.

Proof. It is well known that in this case the action $\rho$ is locally isomorphic to the action $\sigma: \mathfrak{g} \rightarrow D(G)$. By Lemma 2 we obtain that for any $a \in W$ there is a neighborhood $a \in U \subset W$ such that

$$
\begin{aligned}
\{V \in D(U) \mid[V, \rho(\mathfrak{g})]=0\} & \cong \mathfrak{g}, \\
\forall b \in U \forall v \in T_{b} W \quad \exists ! V \in D(U): \mathrm{ev}_{b} V & =v, \quad[V, \rho(\mathfrak{g})]=0 .
\end{aligned}
$$

Since $W$ is connected, this implies the statement of the lemma.

Lemma 4. Let $\mathfrak{g}$ be a (possibly infinite-dimensional) Lie algebra, $W_{1}$ and $W_{2}$ be connected finite-dimensional manifolds, and $\psi: W_{1} \rightarrow W_{2}$ be a morphism of transitive actions $\rho_{i}: \mathfrak{g} \rightarrow D\left(W_{i}\right), i=1,2$. Suppose that $\psi$ is a bundle with connected fibres and the algebra

$$
\mathfrak{s}=\left\{V \in D\left(W_{1}\right) \mid \psi_{*}(V)=0,\left[V, \rho_{1}(\mathfrak{g})\right]=0\right\}
$$

acts freely and transitively on each fibre of $\psi$. Let $\mathfrak{h} \subset \mathfrak{g}$ be the isotropy subalgebra of a point $a \in W_{2}$ with respect to the action $\rho_{2}$. Then all vector fields from $\rho_{1}(\mathfrak{h})$ are tangent to the fibre $F=\psi^{-1}(a) \subset W_{1}$ and the image of the algebra $\rho_{1}(\mathfrak{h})$ in $D(F)$ is isomorphic to $\mathfrak{s}$.

Proof. The fact that all vector fields from $\rho_{1}(\mathfrak{h})$ are tangent to $F$ is obvious. Denote by $\mathfrak{f}$ the image of $\rho_{1}(\mathfrak{h})$ in $D(F)$. The algebra $\{V \in D(F) \mid[V, \mathfrak{s}]=0\}$ includes $\mathfrak{f}$ and is, by Lemma 3 isomorphic to $\mathfrak{s}$. Since $\operatorname{dim} \mathfrak{f} \geq \operatorname{dim} F=\operatorname{dim} \mathfrak{s}$, we obtain

$$
\mathfrak{f}=\{V \in D(F) \mid[V, \mathfrak{s}]=0\} \cong \mathfrak{s} .
$$

1.8. Zero-curvature representations. Let $\mathfrak{g}$ be a Lie algebra over $\mathbb{C}$. Let $\mathcal{E}$ be an open subset of the infinite prolongation of a system of PDEs in two independent variables $x, t$ such that $D_{x}, D_{t}$ are well defined on $\mathcal{E}$.

A pair of functions

$$
M, N: \mathcal{E} \rightarrow \mathfrak{g}
$$

is called a $\mathfrak{g}$-valued zero-curvature representation (ZCR in short) if

$$
D_{x}(N)-D_{t}(M)+[M, N]=0 .
$$

We suppose that all coefficients of the vector-valued functions (40) are admissible (i.e., belong to the structure sheaf).

Then each action $\rho: \mathfrak{g} \rightarrow D(W)$ induces the covering structure in the bundle $\tau: \mathcal{E} \times W \rightarrow \mathcal{E}$ given by

$$
\tilde{D}_{x}=D_{x}+\rho(M), \quad \tilde{D}_{t}=D_{x}+\rho(N) .
$$

Equation (23) for $A=\rho(M)$ and $B=\rho(N)$ follows from (41). 
For a morphism of actions $\psi: W_{1} \rightarrow W_{2}$ the mapping

$$
\text { id } \times \psi: \mathcal{E} \times W_{1} \rightarrow \mathcal{E} \times W_{2}
$$

is a morphism of the corresponding coverings.

Example 6. Let $\mathfrak{g}$ be a finite-dimensional Lie algebra. Clearly, a $\mathfrak{g}$-valued ZCR dependent polynomially on a parameter $\lambda$ can be treated as a ZCR with values in the infinite-dimensional Lie algebra $\mathfrak{g} \otimes_{\mathbb{C}} \mathbb{C}[\lambda]$. Then by the above construction each action of $\mathfrak{g} \otimes_{\mathbb{C}} \mathbb{C}[\lambda]$ determines a covering.

1.9. Translation-invariant coverings. In what follows we mainly consider translation-invariant PDEs (12) such that $F_{\alpha}$ do not depend on the independent variables $x_{i}$. In this case it is convenient to exclude the variables $x_{i}$ from the set of coordinates on $J^{\infty}(\pi)$ and $\mathcal{E}$. That is, admissible functions may depend on (10), but not on $x_{i}$. Besides, in this case we consider total derivative operators (11) without the term $\partial / \partial x_{i}$.

The obtained manifold and the obtained distribution on it are called the translation-invariant infinite prolongation and the translation-invariant Cartan distribution of the translation-invariant system (12) respectively. Differential coverings of the translation-invariant infinite prolongation are called translation-invariant coverings.

Assume that there are two independent variables $x, t$. Then a differential covering (24) is translation-invariant if and only if $a^{j}, b^{j}$ do not depend on $x, t$ either. Making this restriction, we in fact do not loose any coverings, since, according to [7], with arbitrary covering (24) of rank $m$ we can associate the following translationinvariant covering of rank $m+2$

$$
\begin{aligned}
& \frac{\partial v^{1}}{\partial x}=1, \quad \frac{\partial v^{2}}{\partial x}=0, \quad \frac{\partial w^{j}}{\partial x}=a^{j}\left(v^{1}, v^{2}, w^{k}, u_{\sigma}^{i}, \ldots\right), \\
& \frac{\partial v^{1}}{\partial t}=0, \quad \frac{\partial v^{2}}{\partial t}=1, \quad \frac{\partial w^{j}}{\partial t}=b^{j}\left(v^{1}, v^{2}, w^{k}, u_{\sigma}^{i}, \ldots\right)
\end{aligned}
$$

(we replaced $x, t$ by $v^{1}, v^{2}$ in the right-hand side of (24) $)$. The fibres of this covering have the coordinates $v^{1}, v^{2}, w^{1}, \ldots, w^{m}$.

Example 7. Consider a translation-invariant evolution equation

$$
u_{t}=F\left(u, u_{1}, u_{2}, \ldots, u_{p}\right), \quad u_{k}=\frac{\partial^{k} u}{\partial x^{k}}, \quad u=u_{0}
$$

Its translation-invariant infinite prolongation has the coordinates $u_{k}, k \geq 0$. The total derivative operators are written in these coordinates as follows

$$
\begin{aligned}
D_{x} & =\sum_{j \geq 0} u_{j+1} \frac{\partial}{\partial u_{j}} \\
D_{t} & =\sum_{j \geq 0} D_{x}^{j}(F) \frac{\partial}{\partial u_{j}}
\end{aligned}
$$

and span the translation-invariant Cartan distribution.

Let us rewrite the translation-invariance condition in coordinate-free terms.

Recall that a connection in a bundle $\pi: E \rightarrow M$ is given by a distribution $\mathcal{D}$ on $E$ such that for any $a \in E$ the mapping $\pi_{*}: \mathcal{D}_{a} \rightarrow T_{\pi(a)} M$ is an isomorphism 
of vector spaces. Then for each open subset $U \subset M$ we have the natural linear mapping

$$
\nabla: D(U) \rightarrow D\left(\pi^{-1}(U)\right)
$$

that is uniquely defined by the following condition

$$
\forall V \in D(U) \quad \nabla(V) \in \mathcal{D}, \quad \pi_{*}(\nabla(V))=V .
$$

The connection is said to be flat if

$$
\forall V_{1}, V_{2} \in D(U) \quad \nabla\left(\left[V_{1}, V_{2}\right]\right)=\left[\nabla\left(V_{1}\right), \nabla\left(V_{2}\right)\right] .
$$

Consider the natural mapping

$$
\pi_{\infty, 0}: J^{\infty}(\pi) \rightarrow E
$$

arising from (7). Let $Z$ be an open subset of $E$. Recall 1, 9, 10 that for any vector field $X \in D(Z)$ there is a unique vector field $S(X) \in D\left(\pi_{\infty, 0}^{-1}(Z)\right)$ such that

$$
[S(X), \mathcal{C}] \subset \mathcal{C}, \quad\left(\pi_{\infty, 0}\right)_{*}(S(X))=X,
$$

where $\mathcal{C}$ is the Cartan distribution on $J^{\infty}(\pi)$.

Fix a flat connection in the bundle $\pi$. An equation $\mathcal{E} \subset J^{\infty}(\pi)$ is said to be translation-invariant (with respect to this flat connection) if for any vector field $V$ on an open subset of $M$ the vector field $S(\nabla(V))$ is tangent to $\mathcal{E}$.

Vector fields of the form $S(\nabla(V))$ span another distribution $\mathcal{D}^{\prime}$ of $\operatorname{rank} \operatorname{dim} M$ on $J^{\infty}(\pi)$. Let $a \in M$. The submanifold $\mathcal{E}^{\prime}=\mathcal{E} \cap \pi_{\infty}^{-1}(a)$ is the translation-invariant infinite prolongation. To obtain the translation-invariant Cartan distribution $\mathcal{C}_{\mathcal{E}^{\prime}}$ on it, one projects the Cartan distribution $\mathcal{C}_{\mathcal{E}}$ to $\mathcal{E}^{\prime}$ parallel to the distribution $\mathcal{D}^{\prime}$. The obtained distribution $\mathcal{C}_{\mathcal{E}^{\prime}}$ is involutive, but may be singular at some points of $\mathcal{E}^{\prime}$ (e.g., the points $u_{i}=0, i \geq 1$, in Example 7), and we exclude these singular points from the translation-invariant infinite prolongation. It is clear from the next example that locally the structure of the pair $\left(\mathcal{E}^{\prime}, \mathcal{C}_{\mathcal{E}^{\prime}}\right)$ does not depend on $a \in M$.

Example 8. As in Subsection 1.4 let

$$
\pi: \mathbb{C}^{d+n} \rightarrow \mathbb{C}^{n}, \quad\left(x_{1}, \ldots, x_{n}, u^{1}, \ldots, u^{d}\right) \mapsto\left(x_{1}, \ldots, x_{n}\right) .
$$

Consider the flat connection given by $\nabla\left(\partial / \partial x_{i}\right)=\partial / \partial x_{i}$. It is well known that locally any flat connection is isomorphic to this one.

Since we have $S\left(\partial / \partial x_{i}\right)=\partial / \partial x_{i}$, an equation $\mathcal{E} \subset J^{\infty}(\pi)$ is translation-invariant with respect to this flat connection if and only if it can be given by a system (12) such that $F_{\alpha}$ do not depend on $x_{i}$.

1.10. Wahlquist-Estabrook coverings. Consider a translation-invariant evolution equation (42) satisfying $\partial F / \partial u_{p} \neq 0$. In order to describe locally all its translation-invariant coverings, one must solve equation (23) for

$$
\begin{aligned}
A & =\sum_{j=1}^{m} a^{j}\left(w^{1}, \ldots, w^{m}, u, \ldots, u_{k}\right) \frac{\partial}{\partial w^{j}}, \\
B & =\sum_{j=1}^{m} b^{j}\left(w^{1}, \ldots, w^{m}, u, \ldots, u_{k}\right) \frac{\partial}{\partial w^{j}},
\end{aligned}
$$

for arbitrary $k, m \in \mathbb{Z}_{+}$. If $k$ is less than the order $p$ of (42) then the covering is said to be of Wahlquist-Estabrook type.

Consider the following example. 
Proposition 1 (22, 20, 8]). For the KdV equation

$$
u_{t}=u_{3}+u_{1} u, \quad u_{i}=\frac{\partial^{i} u}{\partial x^{i}}
$$

any Wahlquist-Estabrook covering

$$
\begin{gathered}
D_{x} B-D_{t} A+[A, B]=0 \\
A=A\left(w^{1}, \ldots, w^{m}, u, u_{1}, u_{2}\right), \quad B=B\left(w^{1}, \ldots, w^{m}, u, u_{1}, u_{2}\right)
\end{gathered}
$$

is of the form

$$
\begin{gathered}
A=X_{1}+\frac{1}{3} u X_{2}+\frac{1}{6} u^{2} X_{3}, \\
B=\left(\frac{1}{3} u_{2}+\frac{1}{6} u^{2}\right) X_{2}+\left(\frac{1}{9} u^{3}-\frac{1}{6} u_{1}^{2}+\frac{1}{3} u u_{2}\right) X_{3}-X_{4}+ \\
+\frac{1}{3} u\left[X_{1},\left[X_{1}, X_{2}\right]\right]+\frac{1}{18} u^{2}\left[X_{2},\left[X_{1}, X_{2}\right]\right]+\frac{1}{3} u_{1}\left[X_{2}, X_{1}\right],
\end{gathered}
$$

where the vector fields $X_{i}$ depend only on $w^{1}, \ldots, w^{m}$ and are subject to the relations

$$
\begin{gathered}
{\left[X_{1}, X_{3}\right]=\left[X_{2}, X_{3}\right]=\left[X_{1}, X_{4}\right]=\left[X_{2},\left[X_{2},\left[X_{2}, X_{1}\right]\right]\right]=0,} \\
{\left[X_{1},\left[X_{1},\left[X_{2}, X_{1}\right]\right]=\left[X_{4}, X_{2}\right],\left[X_{1},\left[X_{2},\left[X_{2}, X_{1}\right]\right]\right]=\left[X_{1}, X_{2}\right]+\left[X_{4}, X_{3}\right] .\right.}
\end{gathered}
$$

Remark 3. The KdV equation (48) differs from the one described in the introduction, but one is obtained from the other by a suitable scaling transformation $u \mapsto c u$ for some $c \in \mathbb{C}$.

Let $\mathfrak{F}$ be the free Lie algebra generated by the letters $X_{1}, X_{2}, X_{3}, X_{4}$. Let $\mathfrak{L}$ be the quotient of $\mathfrak{F}$ over relations (51), (52). Then formulas (49), (50) determine a ZCR of (48) with values in $\mathfrak{L}$ such that every Wahlquist-Estabrook covering arises from an action of $\mathfrak{L}$ by the construction of Section 1.8 The algebra $\mathfrak{L}$ is called the Wahlquist-Estabrook prolongation algebra of (48).

A similar description of Wahlquist-Estabrook coverings is known for many equations (42) (see, e.g., [2, 20, 8]).

Let us describe the algebra $\mathfrak{L}$ more explicitly. Below for $q \in \mathfrak{s l}_{2}(\mathbb{C})$ and $f(\lambda) \in$ $\mathbb{C}[\lambda]$ we write the element

$$
q \otimes f(\lambda) \in \mathfrak{s l}_{2}(\mathbb{C}) \otimes_{\mathbb{C}} \mathbb{C}[\lambda]
$$

simply as $q f(\lambda)$.

Proposition 2 (19, 20]). The Lie algebra $\mathfrak{L}$ is isomorphic to the direct sum of the Lie algebra $\mathfrak{s l}_{2}(\mathbb{C}) \otimes_{\mathbb{C}} \mathbb{C}[\lambda]$ and the 5-dimensional Heisenberg algebra $H$. The algebra $H$ has a basis

$$
r_{-3}, r_{-1}, r_{0}, r_{1}, r_{3}
$$

with the commutator table $\left[r_{-1}, r_{1}\right]=\left[r_{3}, r_{-3}\right]=r_{0}$, the other commutators being zero. The isomorphism is given by

$$
X_{1}=r_{1}-\frac{1}{2} y+\frac{1}{2} z \lambda, \quad X_{2}=r_{-1}+z, \quad X_{3}=r_{-3}, \quad X_{4}=r_{3}-\frac{1}{2} y \lambda+\frac{1}{2} z \lambda^{2},
$$

where $h, y, z$ is a basis of $\mathfrak{s l}_{2}$ with the relations

$$
[h, y]=2 y, \quad[h, z]=-2 z, \quad[y, z]=h .
$$


Remark 4. One of the main ideas of this paper is to introduce Lie algebras playing similar role for coverings (23), (47) with arbitrary $k$.

The set of coverings of the form (477) is invariant under gauge transformations of the form

$$
w^{i} \mapsto f^{i}\left(w^{1}, \ldots, w^{m}, u, \ldots, u_{k-p}\right) .
$$

In order to define these Lie algebras, we find for coverings (23), (47) a canonical form with respect to the action of gauge transformations (54).

Since for Wahlquist-Estabrook coverings transformations (54) do not depend on $u_{i}, i \geq 0$, all Wahlquist-Estabrook coverings are automatically in the canonical form.

Coverings (23), (47) with arbitrary $k$ were also studied in 3. However, gauge transformations were not considered there. Because of this, the authors of [3] had to impose some additional constraints on vector fields (47).

\section{Analogs of the fundamental Group for Differential COVERINGS}

2.1. An instructive example. To motivate the next constructions, we present a description of some coverings of the $\mathrm{KdV}$ equation

$$
u_{t}=u_{3}+u_{1} u .
$$

The analogous description of all translation-invariant coverings of (55) will be given in Section 4

The operators $D_{x}, D_{t}$ below are given by (43), (44) with $F=u_{3}+u_{1} u$.

Theorem 1. Any translation-invariant covering (23) of the form

$$
A=A\left(w^{1}, \ldots, w^{m}, u, u_{1}, u_{2}, u_{3}\right), \quad B=B\left(w^{1}, \ldots, w^{m}, u, u_{1}, u_{2}, u_{3}\right)
$$

is locally equivalent to a covering of the form

$$
\begin{gathered}
A=X_{1}+\frac{1}{3} u X_{2}+\frac{1}{6} u^{2} X_{3}+f_{1} C, \\
B=\left(\frac{1}{3} u_{2}+\frac{1}{6} u^{2}\right) X_{2}+\left(\frac{1}{9} u^{3}-\frac{1}{6} u_{1}^{2}+\frac{1}{3} u u_{2}\right) X_{3}-X_{4}+ \\
+\frac{1}{3} u\left[X_{1},\left[X_{1}, X_{2}\right]\right]+\frac{1}{18} u^{2}\left[X_{2},\left[X_{1}, X_{2}\right]\right]+\frac{1}{3} u_{1}\left[X_{2}, X_{1}\right]+g_{1} C,
\end{gathered}
$$

where the vector fields $X_{i}, C$ depend only on $w^{1}, \ldots, w^{m}$ and satisfy

$$
\left[C, X_{i}\right]=0, \quad i=1,2,3,4,
$$

in addition to relations (51), (52). Here $\left(f_{1}, g_{1}\right)$ is a conserved current of (55)

$$
\begin{gathered}
f_{1}=u_{1}^{2}-\frac{1}{3} u^{3}, \quad g_{1}=2 u_{1} u_{3}-u_{2}^{2}-u^{2} u_{2}+2 u u_{1}^{2}-\frac{1}{4} u^{4}, \\
D_{t} f_{1}=D_{x} g_{1} .
\end{gathered}
$$

Proof. It is easy to obtain that $A$ does not depend on $u_{2}, u_{3}$ and is a polynomial of degree 2 in $u_{1}$

$$
A=u_{1}^{2} A_{2}\left(w^{1}, \ldots, w^{m}, u\right)+u_{1} A_{1}\left(w^{1}, \ldots, w^{m}, u\right)+A_{0}\left(w^{1}, \ldots, w^{m}, u\right) .
$$

We want to get rid of the term $u_{1} A_{1}$ by switching to a locally gauge equivalent covering. Namely, consider an arbitrary point $u_{i}=a_{i} \in \mathbb{C}, w^{j}=w_{0}^{j} \in \mathbb{C}$ where vector fields (56) are defined. We will find a gauge transformation defined on a neighborhood of this point that kills the term $u_{1} A_{1}$. 
To this end, let

$$
A_{1}\left(w^{1}, \ldots, w^{m}, u\right)=\sum_{j} c^{j}\left(w^{1}, \ldots, w^{m}, u\right) \frac{\partial}{\partial w^{j}} .
$$

Consider the system of ordinary differential equations

$$
\frac{d}{d u} f^{j}\left(w^{1}, \ldots, w^{m}, u\right)=c^{j}\left(f^{1}, \ldots, f^{m}, u\right), \quad j=1, \ldots, m,
$$

dependent on the parameters $w^{1}, \ldots, w^{m}$. Consider its local solution on a neighborhood of the point $u=a_{0}, w^{j}=w_{0}^{j}$ with the initial condition $f^{j}\left(w^{1}, \ldots, w^{m}, a_{0}\right)=$ $w^{j}$. Then the formulas

$$
u_{k} \mapsto u_{k}, \quad w^{j} \mapsto f^{j}\left(w^{1}, \ldots, w^{m}, u\right), \quad k \geq 0, j=1, \ldots, m,
$$

define locally a gauge transformation $\varphi$ such that

$$
\varphi_{*}\left(D_{x}+A\right)=D_{x}+A^{\prime}, \quad \varphi_{*}\left(D_{t}+B\right)=D_{t}+B^{\prime},
$$

where the vector field $A^{\prime}$ is of the form (60) without the linear in $u_{1}$ term (compare with Example 4).

Now it is straightforward to show that the vector fields $A, B$ are of the form (57), (58) with the relations

$$
\begin{gathered}
{\left[X_{2}, X_{3}\right]=\left[X_{1}, X_{4}\right]=\left[C, X_{i}\right]=0, \quad i=1,2,3} \\
{\left[C, X_{4}\right]+\frac{1}{6}\left[X_{1}, X_{3}\right]=0} \\
{\left[C, X_{4}\right]+\frac{1}{3}\left[X_{1}, X_{3}\right]+\frac{1}{6}\left[X_{3},\left[X_{1},\left[X_{1}, X_{2}\right]\right]\right]=\frac{1}{18}\left[X_{2},\left[X_{2},\left[X_{2}, X_{1}\right]\right]\right]} \\
{\left[X_{3},\left[X_{2},\left[X_{1}, X_{2}\right]\right]\right]=0, \quad\left[X_{1},\left[X_{1},\left[X_{2}, X_{1}\right]\right]=\left[X_{4}, X_{2}\right],\right.} \\
{\left[X_{1},\left[X_{2},\left[X_{2}, X_{1}\right]\right]\right]=\left[X_{1}, X_{2}\right]+\left[X_{4}, X_{3}\right] .}
\end{gathered}
$$

From these relations it follows that $\left[X_{1}, X_{3}\right]$ and $\left(\operatorname{ad}^{3} X_{2}\right)\left(X_{1}\right)$ commute with $X_{1}$, $X_{2}$. Now applying $\operatorname{ad}^{2} X_{2}$ to (62) we obtain $\left(\operatorname{ad}^{3} X_{2}\right)\left(X_{1}\right)=0$, which implies (59), (51), (52).

2.2. The definition of the fundamental algebras. Consider a system of PDEs in two independent variables $x, t$. The results of this section are applicable to the following two situations.

(1) The manifold $\mathcal{E}$ is the infinite prolongation defined in Section 1.3 and $\mathcal{C}_{\mathcal{E}}$ is the Cartan distribution on it.

(2) The system of PDEs is translation-invariant, the manifold $\mathcal{E}$ is the translation-invariant infinite prolongation defined in Section 1.9 and $\mathcal{C}_{\mathcal{E}}$ is the translation-invariant Cartan distribution.

However, all examples of this paper belong to the second situation.

Without loss of generality, we can assume $\mathcal{E}$ to be connected. Moreover, we assume that the total derivative operators $D_{x}, D_{t}$ are well defined on $\mathcal{E}$. This is not a big restriction, because most of our results are local and locally this is always the case.

Remark 5. In fact the main Definition 5 below can be readily generalized for PDEs in any number of independent variables. However, since all PDEs considered in this paper are in two independent variables, for the sake of clarity we prefer to give this simplified version. 
Remark 6. Below in this section we use the following notation. For an open subset $\mathcal{E}^{\prime}$ of $\mathcal{E}$ and a finite-dimensional manifold $W$, the mapping

$$
\mathcal{E}^{\prime} \times W \rightarrow \mathcal{E}^{\prime}
$$

is always the projection to the first factor. For a function $f$ on $\mathcal{E}$, its restriction to $\mathcal{E}^{\prime}$ is denoted by the same symbol $f$.

According to Section 1.5 a covering structure in the trivial bundle (63) is uniquely determined by a pair of vector fields $A, B \in D\left(\mathcal{E}^{\prime} \times W\right)$ that are vertical with respect to projection (63) and satisfy relation (23).

We have the natural embedding $D(W) \subset D\left(\mathcal{E}^{\prime} \times W\right)$. A vector field $X \in$ $D\left(\mathcal{E}^{\prime} \times W\right)$ belongs to $D(W)$ if and only if it is vertical with respect to (63) and its coefficients do not depend on coordinates of $\mathcal{E}$.

Inspired by Theorem let us give the following definition.

Definition 5. We say that $\mathcal{E}$ possesses fundamental algebras if there are finite sets $\mathcal{A}_{k}, \mathcal{B}_{k}, k \in \mathbb{Z}_{+}$, of functions on $\mathcal{E}$ satisfying the relations

$$
\mathcal{A}_{k} \subset \mathcal{A}_{k+1}, \quad \mathcal{B}_{k} \subset \mathcal{B}_{k+1} \quad \forall k
$$

such that for any connected open subset $\mathcal{E}_{1}$ of $\mathcal{E}$ the following conditions hold.

(1) Let $\tau: \tilde{\mathcal{E}} \rightarrow \mathcal{E}_{1}$ be a covering of $\mathcal{E}_{1}$. Then for any point $a \in \tilde{\mathcal{E}}$ there are a neighborhood $a \in \tilde{\mathcal{E}}_{1} \subset \tilde{\mathcal{E}}$ and $k \in \mathbb{N}$ such that for $\mathcal{E}_{2}=\tau\left(\tilde{\mathcal{E}}_{1}\right) \subset \mathcal{E}$ the covering $\left.\tau\right|_{\tilde{\mathcal{E}}_{1}}: \tilde{\mathcal{E}}_{1} \rightarrow \mathcal{E}_{2}$ is isomorphic to a covering $\mathcal{E}_{2} \times W \rightarrow \mathcal{E}_{2}$ of the following canonical form

$$
\begin{gathered}
{\left[D_{x}+A, D_{t}+B\right]=0,} \\
A=\sum_{f \in \mathcal{A}_{k}} f M_{f}, \quad B=\sum_{g \in \mathcal{B}_{k}} g N_{g}, \\
M_{f}, N_{g} \in D(W) .
\end{gathered}
$$

(2) Any morphism $\varphi: \mathcal{E}_{1} \times W_{1} \rightarrow \mathcal{E}_{1} \times W_{2}$ between two coverings of the form

$$
\begin{gathered}
\mathcal{E}_{1} \times W_{i} \rightarrow \mathcal{E}_{1}, \quad i=1,2, \\
A^{i}=\sum_{f \in \mathcal{A}_{k}} f M_{f}^{i}, \quad B^{i}=\sum_{g \in \mathcal{B}_{k}} g N_{g}^{i}, \\
{\left[D_{x}+A^{i}, D_{t}+B^{i}\right]=0, \quad M_{f}^{i}, N_{g}^{i} \in D\left(W_{i}\right), \quad i=1,2,}
\end{gathered}
$$

is of the form $\varphi=\mathrm{id} \times \psi$, where

$$
\psi: W_{1} \rightarrow W_{2}, \quad \psi_{*}\left(M_{f}^{1}\right)=M_{f}^{2}, \quad \psi_{*}\left(N_{g}^{1}\right)=N_{g}^{2} .
$$

(3) Let $X \in D\left(\mathcal{E}_{1} \times W\right)$ be a symmetry of a covering $\mathcal{E}_{1} \times W \rightarrow \mathcal{E}_{1}$ given by vector fields

$$
D_{x}+A, D_{t}+B \in D\left(\mathcal{E}_{1} \times W\right)
$$

satisfying (65), 666), 67). Then $X \in D(W)$ and

$$
\left[X, M_{f}\right]=\left[X, N_{g}\right]=0 \quad \forall f \in \mathcal{A}_{k}, \forall g \in \mathcal{B}_{k} .
$$

(4) Consider the manifold $\mathcal{E}_{1} \times W$ with the distribution spanned by $D_{x}+A$, $D_{t}+B$ of the form (65), 667), (67) and let $\mathcal{E}^{\prime}$ be a subequation of it. Then locally $\mathcal{E}^{\prime}$ is of the form $\mathcal{E}_{2} \times W^{\prime}$, where $\mathcal{E}_{2}$ is an open subset of $\mathcal{E}_{1}$ and $W^{\prime}$ is a submanifold of $W$ such that vector fields (67) are tangent to $W^{\prime}$. 
In particular, $\mathcal{E}_{1} \times W$ is irreducible if and only if $W$ is connected and the Lie algebra generated by vector fields (67) acts on $W$ transitively.

In this case fundamental algebras $\mathfrak{f}_{k}$ are defined as follows. Let $\mathfrak{q}_{k}$ be the free Lie algebra generated by the letters $M_{f}, N_{g}$ for $f \in \mathcal{A}_{k}, g \in \mathcal{B}_{k}$. Let us treat (66) as functions on $\mathcal{E}$ with values in $\mathfrak{q}_{k}$. Consider the ideal $I_{k}$ of $\mathfrak{q}_{k}$ generated by the elements

$$
\sum_{g \in \mathcal{B}_{k}} D_{x}(g)(a) N_{g}-\sum_{f \in \mathcal{A}_{k}} D_{t}(f)(a) M_{f}+\sum_{f \in \mathcal{A}_{k}, g \in \mathcal{B}_{k}} f(a) g(a)\left[M_{f}, N_{g}\right], \quad a \in \mathcal{E},
$$

and set $\mathfrak{f}_{k}=\mathfrak{q}_{k} / I_{k}$.

Then (66) becomes an $\mathfrak{f}_{k}$-valued ZCR of $\mathcal{E}$. For an action

$$
\rho: \mathfrak{f}_{k} \rightarrow D(W)
$$

denote by $\tau(\rho)$ the covering $\mathcal{E} \times W \rightarrow \mathcal{E}$ corresponding to (68) by the construction of Section 1.8

From (64) we have the natural epimorphism

$$
p_{k}: \mathfrak{q}_{k} \rightarrow \mathfrak{q}_{k-1}
$$

that maps the generators

$$
M_{f}, \quad N_{g}, \quad f \in \mathcal{A}_{k} \backslash \mathcal{A}_{k-1}, \quad g \in \mathcal{B}_{k} \backslash \mathcal{B}_{k-1},
$$

to zero. It is easily seen that $p_{k}\left(I_{k}\right) \subset I_{k-1}$. Therefore, epimorphisms (69) determine the epimorphisms

$$
\cdots \rightarrow \mathfrak{f}_{k} \rightarrow \mathfrak{f}_{k-1} \rightarrow \cdots \rightarrow \mathfrak{f}_{1} \rightarrow \mathfrak{f}_{0} .
$$

Example 9. From Theorem 1 for the KdV equation (55) we can take

$$
\begin{gathered}
\mathcal{A}_{1}=\left\{1, u, u^{2}, u^{3}, u_{1}^{2}\right\}, \\
\mathcal{B}_{1}=\left\{u^{i_{0}} u_{1}^{i_{1}} u_{2}^{i_{2}} u_{3}^{i_{3}} \mid i_{n} \in \mathbb{Z}_{+}, 0 \leq 2 i_{0}+3 i_{1}+4 i_{2}+5 i_{3} \leq 8\right\} .
\end{gathered}
$$

For example, in this case we have

$$
\begin{aligned}
& M_{u_{1}^{2}}=-3 M_{u^{3}}=\frac{1}{2} N_{u_{1} u_{3}}=-N_{u_{2}^{2}}=C, \\
& M_{u}=N_{u_{2}}=\frac{1}{3} X_{2}, \quad N_{u u_{3}}=N_{u_{1} u_{2}}=0 .
\end{aligned}
$$

The algebra $\mathfrak{f}_{1}$ is isomorphic to the quotient of the free Lie algebra generated by the letters $X_{1}, X_{2}, X_{3}, X_{4}, C$ over relations (51), (52), (59). Formulas (57), (58) determine a ZCR of (55) with values in $\mathfrak{f}_{1}$ such that each covering of the form (56) is equivalent to a covering determined by an action of $\mathfrak{f}_{1}$. The algebra $\mathfrak{f}_{0}$ is isomorphic to the algebra $\mathfrak{L}$ from Section 1.10

For $k>3$, coverings of (55) of the form

$$
A=A\left(w^{1}, \ldots, w^{m}, u, u_{1}, \ldots, u_{k}\right), \quad B=B\left(w^{1}, \ldots, w^{m}, u, u_{1}, \ldots, u_{k}\right)
$$

are determined in a similar way by actions of higher algebras $\mathfrak{f}_{k-2}$, which will be studied in Section 4.2

Remark 7. Consider the identity covering $\mathcal{E} \rightarrow \mathcal{E}$. It has canonical form 665), (66), (67) with $M_{f}=N_{g}=0$ and $W$ equal to a point. From Condition [4 we see that any connected open subset of the equation $\mathcal{E}$ itself must be irreducible. 
Remark 8. Consider an action (68) and let $l \geq k$. Consider the epimorphism $\varphi: \mathfrak{f}_{l} \rightarrow \mathfrak{f}_{k}$ from (71) and the action $\rho \varphi: \mathfrak{f}_{l} \rightarrow D(W)$. By the construction of epimorphisms (71), we have $\tau(\rho \varphi)=\tau(\rho)$.

Therefore, when we consider a finite number of coverings determined by actions

$$
\rho_{i}: \mathfrak{f}_{k_{i}} \rightarrow D\left(W_{i}\right), \quad i=1, \ldots, s,
$$

we can assume that all the actions are defined on the same algebra $\mathfrak{f}_{k}$, where

$$
k=\max \left\{k_{1}, \ldots, k_{s}\right\} .
$$

Below in this section we suppose everywhere that $\mathcal{E}$ possesses fundamental algebras (71) and $\mathcal{E}_{1}$ is a connected open subset of $\mathcal{E}$.

Theorem 2. For any covering $\tau: \tilde{\mathcal{E}} \rightarrow \mathcal{E}_{1}$ each point $a \in \tilde{\mathcal{E}}$ lies in a locally unique irreducible subequation $\tilde{\mathcal{E}}_{a} \subset \tilde{\mathcal{E}}$. The image $\tau\left(\tilde{\mathcal{E}}_{a}\right)$ is open in $\mathcal{E}_{1}$, and $\left.\tau\right|_{\tilde{\mathcal{E}}_{a}}$ is a covering.

Proof. It is sufficient to prove this statement locally. Then we can assume that one has $\tilde{\mathcal{E}}=\mathcal{E}_{1} \times W$ and $\tau=\tau(\rho)$ for some action $\rho: \mathfrak{f}_{k} \rightarrow D(W)$. Let

$$
a=(q, z) \in \mathcal{E}_{1} \times W, \quad q \in \mathcal{E}_{1}, \quad z \in W .
$$

By Proposition 3 below, locally there is a unique submanifold $W^{\prime} \subset W$ such that $z \in W^{\prime}$, all vector fields from $\rho\left(\mathfrak{f}_{k}\right)$ are tangent to $W^{\prime}$, and the induced action on $W^{\prime}$ is transitive. By Condition 40 Definition 5 the submanifold $\tilde{\mathcal{E}}_{a}=\mathcal{E} \times W^{\prime} \subset \tilde{\mathcal{E}}$ is the required irreducible subequation.

Proposition 3 ([13]). Let $\mathfrak{g}$ be an arbitrary Lie algebra over $\mathbb{C}$ and $\rho: \mathfrak{g} \rightarrow D(W)$ be an action of $\mathfrak{g}$ on a complex-analytic manifold $W$. Then for each point $z \in W$ there is submanifold $z \in W^{\prime} \subset W$ such that all vector fields from $\rho(\mathfrak{g})$ are tangent to $W^{\prime}$ and the action of $\mathfrak{g}$ on $W^{\prime}$ is transitive. The submanifold $W^{\prime}$ is locally unique and $\operatorname{dim} W^{\prime}=\operatorname{dim}_{\operatorname{ev}}(\rho(\mathfrak{g}))$.

Consider a covering $\tau: \tilde{\mathcal{E}} \rightarrow \mathcal{E}_{1}$, where $\tilde{\mathcal{E}}$ is connected. Condition 1 of Definition 5 determines locally an action of $\mathfrak{f}_{k}$ on fibres of $\tau$. Due to Condition 2 these local actions produce a well-defined global action

$$
\rho(\tau): \mathfrak{f}_{k} \rightarrow D(\tilde{\mathcal{E}})
$$

such that $\tau_{*} \circ \rho(\tau)=0$.

Theorem 3. The covering $\tau$ is irreducible if and only if the action $\rho(\tau)$ is transitive on each fibre of $\tau$.

Proof. It is sufficient to prove this locally, which is done similarly to the proof of Theorem 2

Theorem 4. Consider two coverings $\tau_{i}: \tilde{\mathcal{E}}^{i} \rightarrow \mathcal{E}_{1}, i=1,2$, and a mapping $\varphi: \tilde{\mathcal{E}}^{1} \rightarrow$ $\tilde{\mathcal{E}}^{2}$ such that the diagram

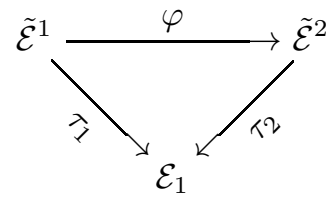

is commutative. 
(1) The mapping $\varphi$ is a morphism of coverings if and only if it is a morphism of the actions $\rho\left(\tau_{1}\right)$ and $\rho\left(\tau_{2}\right)$.

(2) If $\tau_{1}$ and $\tau_{2}$ are irreducible and $\varphi$ is a morphism of coverings then $\varphi\left(\tilde{\mathcal{E}}^{1}\right)$ is open in $\tilde{\mathcal{E}}^{2}$.

Proof. It is sufficient to prove both statements locally.

(1) This follows from Condition 2 of Definition 5

(2) This follows from the previous statement and Lemma 1

Remark 9. Recall that a covering of a connected finite-dimensional manifold $M$ is connected if and only if the corresponding action of $\pi_{1}(M)$ is transitive. Theorem 3 suggests that in PDE geometry irreducible equations play the role of 'connected' objects. Then Theorem 2 is the analog for PDEs of the decomposition into connected components of a topological space.

2.3. Regular coverings and their symmetry algebras. In the present form the analogy of (71) with the topological fundamental group is not sufficiently helpful, because canonical form (66) and the vector fields $M_{f}, N_{g} \in D(W)$ have no invariant (coordinate-free) meaning. In order to recover algebras $\mathfrak{f}_{k}$ in an invariant way, recall that the topological fundamental group can be expressed in terms of automorphism groups of coverings. Studying differential coverings, it is more convenient to consider infinitesimal automorphisms, i.e., symmetries.

From Condition [3 of Definition [5] for each action $\rho: \mathfrak{f}_{k} \rightarrow D(W)$ we obtain

$$
\operatorname{Sym} \tau(\rho)=\left\{v \in D(W) \mid\left[v, \rho\left(\mathfrak{f}_{k}\right)\right]=0\right\} .
$$

Recall that a connected topological covering $\tilde{M} \rightarrow M$ is said to be regular if the action of its automorphism group on $\tilde{M}$ is free and transitive on each fibre. Similarly, we call an irreducible differential covering $\tau: \tilde{\mathcal{E}} \rightarrow \mathcal{E}$ regular if the action on $\tilde{\mathcal{E}}$ of the algebra $\operatorname{Sym} \tau$ is free and transitive on each fibre of $\tau$. In particular, $\tau$ is the quotient map with respect to this action, and $\operatorname{dim} \operatorname{Sym} \tau=\operatorname{rank} \tau$.

Theorem 5. A covering $\tau: \tilde{\mathcal{E}} \rightarrow \mathcal{E}_{1}$ is regular if and only if the action on $\tilde{\mathcal{E}}$ of the subalgebra $\rho(\tau)\left(\mathfrak{f}_{k}\right) \subset D(\tilde{\mathcal{E}})$ is free and transitive on each fibre of $\tau$. In this case one has $\operatorname{dim} \rho(\tau)\left(\mathfrak{f}_{k}\right)=\operatorname{rank} \tau$ and $\operatorname{Sym} \tau \cong \rho(\tau)\left(\mathfrak{f}_{k}\right)$.

Proof. It is sufficient to prove this locally, and the local version follows from (72) and Lemma 3

Each ideal $\mathfrak{i}$ of $\mathfrak{f}_{k}$ with codim $\mathfrak{i}<\infty$ determines a regular covering as follows. Consider the canonical epimorphism $\psi: \mathfrak{f}_{k} \rightarrow \mathfrak{f}_{k} / \mathfrak{i}$. Let $\sigma: \mathfrak{f}_{k} / \mathfrak{i} \rightarrow D(G)$ be the natural action by right invariant vector fields on the simply connected Lie group $G$ whose Lie algebra is the finite-dimensional algebra $\mathfrak{f}_{k} / \mathfrak{i}$. For any open subset $U \subset G$ we have the transitive action $\sigma \psi: \mathfrak{f}_{k} \rightarrow D(U)$. By Theorem [5 the corresponding covering $\tau(\sigma \psi)$ is regular, and every regular covering is locally isomorphic to a covering of this form. By Remark 8 if $\mathfrak{i}_{n} \subset \mathfrak{f}_{n}, n \geq k$, is the preimage of $\mathfrak{i}$ under epimorphism (71) then $\mathfrak{f}_{n} / \mathfrak{i}_{n} \cong \mathfrak{f}_{k} / \mathfrak{i}$ and the corresponding regular coverings are also isomorphic.

Let $\mathfrak{i}_{1}, \mathfrak{i}_{2} \subset \mathfrak{f}_{k}$ be two ideals of finite codimension. Consider the simply connected Lie groups $G_{1}, G_{2}$ associated with the Lie algebras $\mathfrak{f}_{k} / \mathfrak{i}_{1}, \mathfrak{f}_{k} / \mathfrak{i}_{2}$. Let $U_{i} \subset G_{i}, i=$ 1,2 , be connected open subsets. 
Suppose that the corresponding regular coverings are connected by a morphism $\varphi$

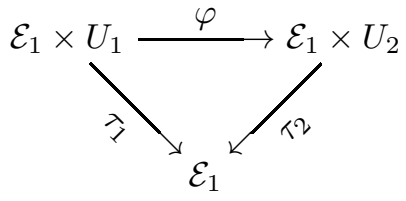

From Condition 2 of Definition 5 it follows that $\mathfrak{i}_{1} \subset \mathfrak{i}_{2}$ and $\varphi=$ id $\times \psi$, where $\psi: U_{1} \rightarrow U_{2}$ is a morphism of actions of $\mathfrak{f}_{k}$. By Theorem $\left[\right.$ we have $\operatorname{Sym} \tau_{i} \cong$ $\mathfrak{f}_{k} / \mathfrak{i}_{i}, i=1,2$. The mapping

$$
\varphi: \mathcal{E}_{1} \times U_{1} \rightarrow \varphi\left(\mathcal{E}_{1} \times U_{1}\right)=\mathcal{E}_{1} \times \psi\left(U_{1}\right)
$$

is the quotient mapping with respect to the action of the subalgebra $\mathfrak{i}_{2} / \mathfrak{i}_{1} \subset \operatorname{Sym} \tau_{1}$ on the manifold $\mathcal{E}_{1} \times U_{1}$.

Similarly to Theorem 4 this local description of regular coverings and morphisms connecting them implies the following global result.

Theorem 6. Consider two regular coverings $\tau_{i}: \tilde{\mathcal{E}}^{i} \rightarrow \mathcal{E}_{1}, i=1,2$, and let

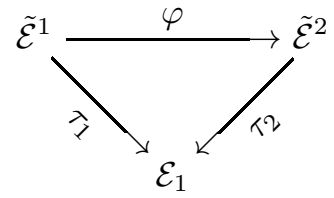

be a morphism of them. Then there is $k \in \mathbb{N}$ and two ideals $\mathfrak{i}_{1}, \mathfrak{i}_{2}$ of $\mathfrak{f}_{k}$ of finite codimension such that

- one has

$$
\operatorname{Sym} \tau_{i}=\mathfrak{f}_{k} / \mathfrak{i}_{i}, \quad i=1,2,
$$

- we have $\mathfrak{i}_{1} \subset \mathfrak{i}_{2}$, the subset $\varphi\left(\tilde{\mathcal{E}}^{1}\right)$ is open in $\tilde{\mathcal{E}}^{2}$, and the mapping $\varphi: \tilde{\mathcal{E}}^{1} \rightarrow$ $\varphi\left(\tilde{\mathcal{E}}^{1}\right)$ is the quotient mapping with respect to the action of the subalgebra $\mathfrak{i}_{2} / \mathfrak{i}_{1} \subset \operatorname{Sym} \tau_{1}$ on the manifold $\tilde{\mathcal{E}}^{1}$,

- the differential $\varphi_{*}$ of $\varphi$ induces an epimorphism of algebras Sym $\tau_{1} \rightarrow$ Sym $\tau_{2}$. In terms of isomorphisms (73) it is the natural epimorphism $\mathfrak{f}_{k} / \mathfrak{i}_{1} \rightarrow \mathfrak{f}_{k} / \mathfrak{i}_{2}$ corresponding to the inclusion $\mathfrak{i}_{1} \subset \mathfrak{i}_{2}$.

In contrast to fundamental algebras (171), the system of symmetry algebras of regular coverings is a coordinate-free canonical invariant of a system of PDEs, since symmetry algebras are coordinate-independent objects. Thus we recover in an invariant way not algebras (71) themselves, but all their finite-dimensional quotients.

2.4. Quasi-finite Lie algebras. We present here some results on Lie algebras.

Definition 6. A Lie algebra $\mathfrak{g}$ is said to be quasi-finite if for any subalgebra $\mathfrak{h} \subset \mathfrak{g}$ of finite codimension there is an ideal of $\mathfrak{g}$ that is of finite codimension and is contained in $\mathfrak{h}$.

Theorem 7. Let $\mathfrak{g}$ be a quasi-finite algebra. Then for any transitive action $\rho: \mathfrak{g} \rightarrow$ $D(W)$ on a connected finite-dimensional manifold $W$ the algebra $\rho(\mathfrak{g})$ is finitedimensional. 
Proof. Let $a \in W$ and $\mathfrak{h} \subset \mathfrak{g}$ be the isotropy subalgebra of $a$. Since $\mathfrak{g}$ is quasi-finite and $\operatorname{codim} \mathfrak{h}=\operatorname{dim} W<\infty$, there is an ideal $\mathfrak{i}$ of $\mathfrak{g}$ such that $\mathfrak{i} \subset \mathfrak{h}$ and codim $\mathfrak{i}<\infty$. It is well known that in the complex-analytic situation the image $\rho(\mathfrak{h})$ of the isotropy subalgebra cannot contain any nontrivial ideal of $\rho(\mathfrak{g})$. Therefore, $\rho(\mathfrak{i})=0$ and

$$
\operatorname{dim} \rho(\mathfrak{g}) \leq \operatorname{codim} \mathfrak{i}<\infty .
$$

Theorem 8. Let $\mathfrak{g}$ be a quasi-finite Lie algebra and $\rho: \tilde{\mathfrak{g}} \rightarrow \mathfrak{g}$ be an epimorphism such that

$$
[\operatorname{ker} \rho, \tilde{\mathfrak{g}}]=0
$$

(that is, $\tilde{\mathfrak{g}}$ is a central extension of $\mathfrak{g})$. Then $\tilde{\mathfrak{g}}$ is also quasi-finite.

Proof. Let $\mathfrak{h} \subset \tilde{\mathfrak{g}}$ be a subalgebra of finite codimension. Clearly, the subset

$$
\left.\mathfrak{h}_{1}=\{a \in \mathfrak{h} \mid[\tilde{\mathfrak{g}}, a] \subset \mathfrak{h}]\right\}
$$

is also a subalgebra of finite codimension. By assumption, there is an ideal $\mathfrak{i}$ of $\mathfrak{g}$ that is of finite codimension and is contained in $\rho\left(\mathfrak{h}_{1}\right)$.

The subspace $\rho^{-1}(\mathfrak{i}) \cap \mathfrak{h}$ is of finite codimension and is contained in $\mathfrak{h}$. Let us prove that $\rho^{-1}(\mathfrak{i}) \cap \mathfrak{h}$ is an ideal of $\tilde{\mathfrak{g}}$.

Let $a \in \rho^{-1}(\mathfrak{i}) \cap \mathfrak{h}$ and $v \in \tilde{\mathfrak{g}}$. Then $a=h+z$, where $h \in \mathfrak{h}_{1}$ and $z \in \operatorname{ker} \rho$. Combining (74) and (75), we obtain $[v, a] \in \mathfrak{h}$. Besides, since $\rho^{-1}(\mathfrak{i})$ is an ideal of $\tilde{\mathfrak{g}}$, we have $[v, a] \in \rho^{-1}(\mathfrak{i})$. Therefore, $[v, a] \in \rho^{-1}(\mathfrak{i}) \cap \mathfrak{h}$.

Let $\mathfrak{g}$ be a Lie algebra over $\mathbb{C}$ and $\mathcal{A}$ be a commutative associative algebra over $\mathbb{C}$. Then the space $\mathfrak{g} \otimes_{\mathbb{C}} \mathcal{A}$ has the following natural Lie algebra structure

$$
\left[g_{1} \otimes a_{1}, g_{2} \otimes a_{2}\right]=\left[g_{1}, g_{2}\right] \otimes a_{1} a_{2}, \quad g_{1}, g_{2} \in \mathfrak{g}, a_{1}, a_{2} \in \mathcal{A} .
$$

Theorem 9. If $\mathfrak{g}$ is finite-dimensional and semisimple then the Lie algebra $\mathfrak{g} \otimes_{\mathbb{C}} \mathcal{A}$ is quasi-finite.

Proof. Let $\mathfrak{h} \subset \mathfrak{g} \otimes_{\mathbb{C}} \mathcal{A}$ be a subalgebra of finite codimension. Then the subspace

$$
Z=\{f \in \mathcal{A} \mid \mathfrak{g} \otimes f \subset \mathfrak{h}\}
$$

is of finite codimension in $\mathcal{A}$. Since $[\mathfrak{g}, \mathfrak{g}]=\mathfrak{g}$, the subspace $Z$ is a subring of $\mathcal{A}$. The subspace $Z^{\prime}=\{f \in Z \mid f \mathcal{A} \subset Z\}$ is of finite codimension and is an ideal of the ring $\mathcal{A}$. Therefore, the subspace $\mathfrak{g} \otimes Z^{\prime}$ is an ideal of $\mathfrak{g} \otimes \mathcal{A}$ of finite codimension, and from (77) we have $\mathfrak{g} \otimes Z^{\prime} \subset \mathfrak{h}$.

2.5. Local structure of irreducible coverings. Below we suppose that algebras (71) are quasi-finite.

Consider a subalgebra $\mathfrak{h} \subset \mathfrak{f}_{k}$ of finite codimension. Let $\mathfrak{i}(\mathfrak{h})$ be the maximal ideal of $\mathfrak{f}_{k}$ that is contained in $\mathfrak{h}$. Since $\mathfrak{f}_{k}$ is quasi-finite, we have codim $\mathfrak{i}(\mathfrak{h})<\infty$.

Let $G$ be the simply connected Lie group whose Lie algebra is $\mathfrak{f}_{k} / \mathfrak{i}(\mathfrak{h})$ and $H \subset$ $G$ be the connected Lie subgroup whose Lie subalgebra is $\mathfrak{h} / \mathfrak{i}(\mathfrak{h})$. According to Section 1.7 the algebra $\mathfrak{f}_{k}$ acts on $G$ by right invariant vector fields, which are projected also to the quotient space $G / H$. Denote by $\sigma\left(\mathfrak{f}_{k}, \mathfrak{h}\right)$ the arising transitive action of $\mathfrak{f}_{k}$ on the manifold $W\left(\mathfrak{f}_{k}, \mathfrak{h}\right)=G / H$. We have $\operatorname{ker} \sigma\left(\mathfrak{f}_{k}, \mathfrak{h}\right)=\mathfrak{i}(\mathfrak{h})$. 
Remark 10. Let $G$ be a Lie group associated with a Lie algebra $\mathfrak{g}$. Generally, not for every subalgebra $\mathfrak{h} \subset \mathfrak{g}$ there is a Lie subgroup whose Lie subalgebra is $\mathfrak{h}$. However, for us it is sufficient to consider the local Lie subgroup, which always exists. In this case the symbol $G / H$ denotes the quotient space not of the whole group $G$, but of some neighborhood of the unity element.

As above, let $\mathcal{E}_{1}$ be a connected open subset of $\mathcal{E}$. Consider the manifold $\mathcal{E}_{1}\left(\mathfrak{f}_{k}, \mathfrak{h}\right)=\mathcal{E}_{1} \times W\left(\mathfrak{f}_{k}, \mathfrak{h}\right)$ and the covering

$$
\tau\left(\mathfrak{f}_{k}, \mathfrak{h}\right): \mathcal{E}_{1}\left(\mathfrak{f}_{k}, \mathfrak{h}\right) \rightarrow \mathcal{E}_{1}
$$

corresponding to the action $\sigma\left(\mathfrak{f}_{k}, \mathfrak{h}\right)$ of $\mathfrak{f}_{k}$.

Theorem 10. The following statements hold.

(1) Every irreducible covering $\tau$ of $\mathcal{E}_{1}$ is locally isomorphic to a covering $\tau\left(\mathfrak{f}_{k}, \mathfrak{h}\right)$ for some $k \in \mathbb{N}$ and $\mathfrak{h} \subset \mathfrak{f}_{k}$.

(2) We have

$$
\operatorname{Sym} \tau\left(\mathfrak{f}_{k}, \mathfrak{h}\right) \cong \mathfrak{n}(\mathfrak{h}) / \mathfrak{h},
$$

where

$$
\mathfrak{n}(\mathfrak{h})=\left\{v \in \mathfrak{f}_{k} \mid[v, \mathfrak{h}] \subset \mathfrak{h}\right\} .
$$

(3) The covering $\tau\left(\mathfrak{f}_{k}, \mathfrak{h}\right)$ is regular if and only if $\mathfrak{h}$ is an ideal of $\mathfrak{f}_{k}$.

Proof. (1) By Conditions 1 1 and 4 of Definition 5 locally we have $\tau=\tau(\rho)$ for some transitive action $\rho: \mathfrak{f}_{k} \rightarrow D(W)$. Let $a \in W$ and consider the isotropy subalgebra $\mathfrak{h} \subset \mathfrak{f}_{k}$ of $a$. By Theorem $\mathbf{7}$ the actions $\rho$ and $\sigma\left(\mathfrak{f}_{k}, \mathfrak{h}\right)$ are locally isomorphic. Then the coverings $\tau(\rho)$ and $\tau\left(\mathfrak{f}_{k}, \mathfrak{h}\right)$ are locally isomorphic as well.

(2) Formulas (78) and (79) follow from formula (72) and Lemma 2

(3) This follows from Theorem 5

Recall that for any connected topological covering $\tau: \tilde{M} \rightarrow M$ there is a commutative diagram of coverings

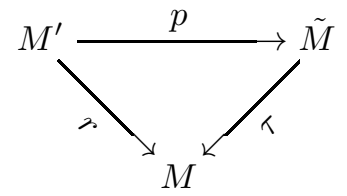

where $r$ is regular and $p$ is the quotient mapping with respect to the action of some automorphism subgroup of $r$. Let us construct an analog of diagram (80) for differential coverings.

Theorem 11. Any irreducible covering $\tau: \tilde{\mathcal{E}} \rightarrow \mathcal{E}_{1}$ is locally included in a commutative diagram of irreducible coverings

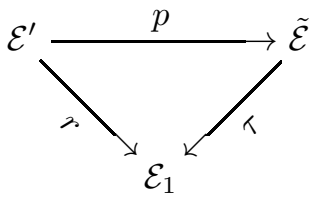

such that the following assertions hold.

(1) The covering $r$ is regular. 
(2) The covering $p$ is the quotient morphism with respect to the action on $\mathcal{E}^{\prime}$ of some subalgebra $\mathfrak{h}$ of Sym $r$.

(3) The algebra $\operatorname{Sym} \tau$ coincides with the quotient $\mathfrak{n} / \mathfrak{h}$, where

$$
\mathfrak{n}=\{v \in \operatorname{Sym} r \mid[v, \mathfrak{h}] \subset \mathfrak{h}\},
$$

and the action of $\mathfrak{n} / \mathfrak{h}$ on $\tilde{\mathcal{E}}$ is induced by the action of $\mathfrak{n}$ on $\mathcal{E}^{\prime}$.

Proof. By Theorem 10 (1), it is sufficient to prove the statements for $\tau=\tau\left(\mathfrak{f}_{k}, \mathfrak{h}_{1}\right)$, where $\mathfrak{h}_{1}$ is a subalgebra of $\mathfrak{f}_{k}$ of finite codimension.

Recall that $\mathfrak{i}\left(\mathfrak{h}_{1}\right)$ is the maximal ideal of $\mathfrak{f}_{k}$ that is contained in $\mathfrak{h}_{1}$. By Theorem 10 (3), the covering $r=\tau\left(\mathfrak{f}_{k}, \mathfrak{i}\left(\mathfrak{h}_{1}\right)\right)$ is regular. The inclusion of Lie algebras

$$
\mathfrak{i}\left(\mathfrak{h}_{1}\right) \subset \mathfrak{h}_{1} \subset \mathfrak{f}_{k}
$$

determines a surjective morphism

$$
W\left(\mathfrak{f}_{k}, \mathfrak{i}\left(\mathfrak{h}_{1}\right)\right) \rightarrow W\left(\mathfrak{f}_{k}, \mathfrak{h}_{1}\right)
$$

of actions of $\mathfrak{f}_{k}$, which determines the surjective morphism

$$
p: \mathcal{E}_{1}\left(\mathfrak{f}_{k}, \mathfrak{i}\left(\mathfrak{h}_{1}\right)\right) \rightarrow \mathcal{E}_{1}\left(\mathfrak{f}_{k}, \mathfrak{h}_{1}\right), \quad \tau\left(\mathfrak{f}_{k}, \mathfrak{h}_{1}\right) \circ p=\tau\left(\mathfrak{f}_{k}, \mathfrak{i}\left(\mathfrak{h}_{1}\right)\right),
$$

of the corresponding coverings.

By formulas (78) and (79), one has $\operatorname{Sym} r=\mathfrak{f}_{k} / \mathfrak{i}\left(\mathfrak{h}_{1}\right)$. By construction, the morphism $p$ is the quotient map with respect to the action of $\mathfrak{h}=\mathfrak{h}_{1} / \mathfrak{i}\left(\mathfrak{h}_{1}\right) \subset \operatorname{Sym} r$ on the manifold $\mathcal{E}_{1}\left(\mathfrak{f}_{k}, \mathfrak{i}\left(\mathfrak{h}_{1}\right)\right)$. Finally, the last statement of the theorem follows from formulas (78), (79).

For a subalgebra $\mathfrak{h}$ of $\mathfrak{f}_{k}$ of finite codimension, denote by $\mathfrak{h}_{l}$ the preimage of $\mathfrak{h}$ in $\mathfrak{f}_{l}, l \geq k$, under epimorphisms (71). By Remark 8 , one obtains

$$
\mathcal{E}_{1}\left(\mathfrak{f}_{l}, \mathfrak{h}_{l}\right) \cong \mathcal{E}_{1}\left(\mathfrak{f}_{k}, \mathfrak{h}\right), \quad \tau\left(\mathfrak{f}_{l}, \mathfrak{h}_{l}\right) \cong \tau\left(\mathfrak{f}_{k}, \mathfrak{h}\right) \quad \forall l \geq k
$$

If $\tilde{\mathfrak{h}} \subset \mathfrak{h}_{l}$ is a subalgebra of finite codimension then we have the natural surjective morphism $W\left(\mathfrak{f}_{l}, \tilde{\mathfrak{h}}\right) \rightarrow W\left(\mathfrak{f}_{l}, \mathfrak{h}_{l}\right)$ of actions of $\mathfrak{f}_{l}$, which determines a covering

$$
\tau(\mathfrak{h}, \tilde{\mathfrak{h}}): \mathcal{E}_{1}\left(\mathfrak{f}_{l}, \tilde{\mathfrak{h}}\right) \rightarrow \mathcal{E}_{1}\left(\mathfrak{f}_{l}, \mathfrak{h}_{l}\right) \cong \mathcal{E}_{1}\left(\mathfrak{f}_{k}, \mathfrak{h}\right) .
$$

Let $\mathfrak{i}$ be an ideal of $\mathfrak{h}_{l}$ with codim $\mathfrak{i}<\infty$ (but not necessarily an ideal of $\mathfrak{f}_{l}$ ). By formulas (78), (79), the covering

$$
\tau(\mathfrak{h}, \mathfrak{i}): \mathcal{E}_{1}\left(\mathfrak{f}_{l}, \mathfrak{i}\right) \rightarrow \mathcal{E}_{1}\left(\mathfrak{f}_{k}, \mathfrak{h}\right)
$$

is regular and $\operatorname{Sym} \tau(\mathfrak{h}, \mathfrak{i}) \cong \mathfrak{h}_{l} / \mathfrak{i}$. The following theorem shows that locally every regular covering of $\mathcal{E}_{1}\left(\mathfrak{f}_{k}, \mathfrak{h}\right)$ is of this form.

Theorem 12. Consider an irreducible covering $\tau: \tilde{\mathcal{E}} \rightarrow \mathcal{E}_{1}$ and the corresponding action $\rho(\tau): \mathfrak{f}_{k} \rightarrow D(\tilde{\mathcal{E}})$. Let $a \in \tilde{\mathcal{E}}$ and $\mathfrak{h} \subset \mathfrak{f}_{k}$ be the isotropy subalgebra of a.

Then for any connected neighborhood $\tilde{\mathcal{E}}^{\prime} \subset \tilde{\mathcal{E}}$ of a the symmetry algebra of any regular covering over $\tilde{\mathcal{E}}^{\prime}$ is isomorphic to a finite-dimensional quotient of $\mathfrak{h}_{l}$ for some $l \geq k$.

And vice versa, for any $l \geq k$ and any ideal $\mathfrak{i}$ of $\mathfrak{h}_{l}$ of finite codimension there is a regular covering $\tau^{\prime}$ over a neighborhood of a such that $\operatorname{Sym} \tau^{\prime}=\mathfrak{h}_{l} / \mathfrak{i}$. 
Proof. Let $\tau^{\prime}: \mathcal{E}^{\prime \prime} \rightarrow \tilde{\mathcal{E}}^{\prime}$ be a regular covering. Consider the connected open subset $\mathcal{E}_{2}=\tau\left(\tilde{\mathcal{E}}^{\prime}\right)$ of $\mathcal{E}_{1}$ and the commutative diagram of coverings

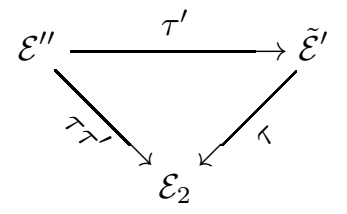

Since the question is essentially local, we can assume that the above diagram is of the form

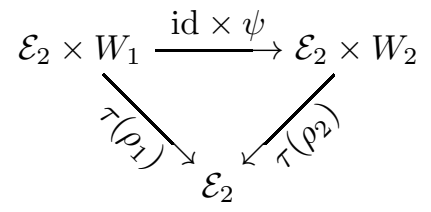

where $\rho_{i}: \mathfrak{f}_{l} \rightarrow D\left(W_{i}\right), i=1,2$, are transitive actions for some $l \geq k$ and $\psi: W_{1} \rightarrow$ $W_{2}$ is a morphism of actions. The point $a$ is of the form $a=(q, z), q \in \mathcal{E}_{2}, z \in W_{2}$. The algebra $h_{l}$ is the isotropy algebra of $z$ with respect to the action $\rho_{2}$. Then the first statement of the theorem follows from Lemma 4 for $\mathfrak{g}=\mathfrak{f}_{l}$.

The second statement of the theorem follows from construction (81).

This theorem is the analog of the fact that for a connected topological covering $\tilde{M} \rightarrow M$ one has $\pi_{1}(\tilde{M}) \subset \pi_{1}(M)$.

Since $\mathfrak{g}$ in Lemma 4 is allowed to be infinite-dimensional, the first statement of Theorem 12 holds even if algebras (71) are not quasi-finite.

Theorem 13. In the notation of Theorem 12, the symmetry algebra of any regular covering over $\tilde{\mathcal{E}}^{\prime}$ is isomorphic to a finite-dimensional quotient of $\mathfrak{h}_{l}$ for some $l \geq k$ even if the fundamental algebras are not quasi-finite.

2.6. Necessary conditions for existence of Bäcklund transformations. Consider two systems of PDEs

$$
\mathcal{E}_{i}=\left\{F_{\alpha}^{i}\left(x, t, u^{i 1}, \ldots, u^{i d_{i}}, \frac{\partial^{p+q} u^{i j}}{\partial x^{p} \partial t^{q}}, \ldots\right)=0, \alpha=1, \ldots, s_{i}\right\}, \quad i=1,2,
$$

A Bäcklund transformation between $\mathcal{E}_{1}$ and $\mathcal{E}_{2}$ is given by another system

$$
F_{\alpha}\left(x, t, v^{1}, \ldots, v^{d}, \frac{\partial^{p+q} v^{j}}{\partial x^{p} \partial t^{q}}, \ldots\right)=0, \quad \alpha=1, \ldots, s .
$$

and two mappings

$$
u^{i j}=\varphi^{i j}\left(x, t, v^{1}, \ldots, v^{d}, \frac{\partial^{p+q} v^{l}}{\partial x^{p} \partial t^{q}}, \ldots\right), \quad j=1, \ldots, d_{i}, i=1,2,
$$

such that for each $i=1,2$ one has

- for each local solution $v^{1}(x, t), \ldots, v^{d}(x, t)$ of 833) functions (84) form a local solution of (82),

- for each local solution $u^{i 1}(x, t), \ldots, u^{i d_{i}}(x, t)$ of (82) the system that consists of equations (83) and (84) is consistent and possesses locally a general solution

$$
v^{1}\left(x, t, c_{1}, \ldots, c_{r_{i}}\right), \ldots, v^{d}\left(x, t, c_{1}, \ldots, c_{r_{i}}\right)
$$

dependent on a finite number of complex parameters $c_{1}, \ldots, c_{r_{i}}$. 
Similarly to Section 1.6 these conditions mean by definition that the infinite prolongation $\tilde{\mathcal{E}}$ of 83 covers both $\mathcal{E}_{1}$ and $\mathcal{E}_{2}$

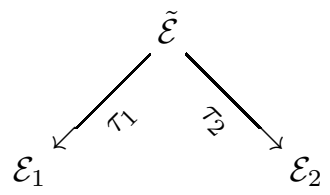

where the covering $\tau_{i}$ is of rank $r_{i}$. We allow $\mathcal{E}_{i}$ to be not the whole infinite prolongation, but some nonempty open subset of it.

If systems (82) are translation-invariant then we can make (83) translationinvariant as well using the trick from Section 1.9]: replace $x, t$ in $F_{\alpha}$ and $\varphi^{i j}$ by the new dependent variables $w^{1}, w^{2}$ respectively and add to (83) the following equations

$$
\frac{\partial w^{1}}{\partial x}=\frac{\partial w^{2}}{\partial t}=1, \quad \frac{\partial w^{1}}{\partial t}=\frac{\partial w^{2}}{\partial x}=0 .
$$

After this substitution coverings [85] become translation-invariant.

Example 10. Consider two different coverings from the modified KdV equation to the KdV equation

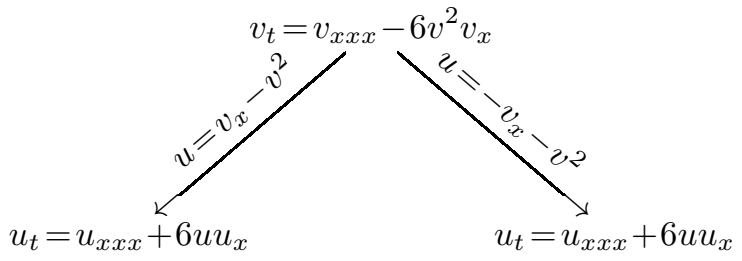

This diagram presents a Bäcklund auto-transformation of the KdV equation. See, e.g, 15, 16] for more examples of Bäcklund transformations.

Theorem 14. Suppose that two systems $\mathcal{E}_{i}, i=1,2$, possess fundamental algebras

$$
\cdots \rightarrow \mathfrak{f}_{k+1}^{i} \rightarrow \mathfrak{f}_{k}^{i} \rightarrow \cdots \rightarrow \mathfrak{f}_{1}^{i} \rightarrow \mathfrak{f}_{0}^{i}, \quad i=1,2,
$$

and the algebras $\mathfrak{f}_{k}^{1}$ are quasi-finite. Let $\mathfrak{g}$ be a finite-dimensional Lie algebra. Suppose that for any $k_{1}, k_{2} \in \mathbb{Z}_{+}$and any subalgebras

$$
\mathfrak{h}^{i} \subset \mathfrak{f}_{k_{i}}^{i}, \quad \operatorname{codim} \mathfrak{h}^{i}<\infty, \quad i=1,2,
$$

there is an epimorphism $\mathfrak{h}^{1} \rightarrow \mathfrak{g}$, but there is no epimorphism $\mathfrak{h}^{2} \rightarrow \mathfrak{g}$. Then there is no Bäcklund transformation between $\mathcal{E}_{1}$ and $\mathcal{E}_{2}$.

Proof. Suppose that there is a Bäcklund transformation. By the above construction, it determines a diagram (85) of coverings. Let $a \in \tilde{\mathcal{E}}$. By Theorem 2 locally there is a unique irreducible subequation $\tilde{\mathcal{E}}_{a} \subset \tilde{\mathcal{E}}$ that contains $a$. The subset $\tau_{i}\left(\tilde{\mathcal{E}}_{a}\right)$ is open in $\mathcal{E}_{i}$, and the coverings

$$
\left.\tau_{i}\right|_{\tilde{\mathcal{E}}_{a}}: \tilde{\mathcal{E}}_{a} \rightarrow \tau_{i}\left(\tilde{\mathcal{E}}_{a}\right), \quad i=1,2,
$$

are irreducible. Consider the action

$$
\rho\left(\left.\tau_{1}\right|_{\tilde{\mathcal{E}}_{a}}\right): \mathfrak{f}_{k}^{1} \rightarrow D\left(\tilde{\mathcal{E}}_{a}\right)
$$

and let $\mathfrak{h}^{1} \subset \mathfrak{f}_{k}^{1}$ be the isotropy subalgebra of $a$. By Theorem [12] an epimorphism $\mathfrak{h}^{1} \rightarrow \mathfrak{g}$ implies that over a connected neighborhood of $a \in \tilde{\mathcal{E}}_{a}$ there is a regular 
covering with symmetry algebra equal to $\mathfrak{g}$. Applying Theorem 13 to this regular covering and the covering $\left.\tau_{2}\right|_{\tilde{\mathcal{E}}_{a}}$, we obtain that $\mathfrak{g}$ is isomorphic to a quotient of some subalgebra $\mathfrak{h}^{2}$ of $\mathfrak{f}_{l}^{2}$ of finite codimension. Thus we get a contradiction.

\section{Coverings of SCAlar EVolution Equations}

In this section we prove some technical results, which will be needed in Sections 4 and 5 Consider a translation-invariant evolution equation

$$
\begin{gathered}
u_{t}=F\left(u, u_{1}, u_{2}, \ldots, u_{p}\right), \quad \frac{\partial F}{\partial u_{p}} \neq 0, \\
u_{i}=\frac{\partial^{i} u}{\partial x^{i}}, \quad u=u_{0} .
\end{gathered}
$$

Let $\mathcal{E}$ be a connected open subset of the translation-invariant infinite prolongation of this equation described in Example $\mathbf{7}$

Let $W$ be a connected open subset of $\mathbb{C}^{m}$ with coordinates $w^{1}, \ldots, w^{m}$ and

$$
u_{i}=a_{i} \in \mathbb{C}, \quad i=0,1, \ldots,
$$

be a point of $\mathcal{E}$. Consider a covering $\mathcal{E} \times W \rightarrow \mathcal{E}$ given by vector fields

$$
\begin{gathered}
A=\sum_{j=1}^{m} a^{j}\left(w^{1}, \ldots, w^{m}, u, \ldots, u_{k}\right) \frac{\partial}{\partial w^{j}}, \\
B=\sum_{j=1}^{m} b^{j}\left(w^{1}, \ldots, w^{m}, u, \ldots, u_{k}\right) \frac{\partial}{\partial w^{j}} \\
D_{x} B-D_{t} A+[A, B]=0 .
\end{gathered}
$$

Below we sometimes omit the dependence on the coordinates $w^{i}$ in vector fields on $\mathcal{E} \times W$.

Remark 11. Below in this section we say that locally there is a gauge transformation with certain properties if for any $w \in W$ a gauge transformation with these properties exists on a neighborhood of the point $(a, w) \in \mathcal{E} \times W$, where $a$ is the fixed point (89) of $\mathcal{E}$.

Lemma 5. We have

$$
\frac{\partial A}{\partial u_{s}}=0 \quad \forall s>k-p+1 .
$$

Moreover, locally there is a gauge transformation

$$
w^{i} \mapsto f^{i}\left(w^{1}, \ldots, w^{m}, u, \ldots, u_{k-p}\right), \quad i=1, \ldots, m,
$$

such that the transformed vector field $D_{x}+A$ satisfies for all $s \geq 1$

$$
\frac{\partial A}{\partial u_{s}}\left(u, \ldots, u_{s-1}, a_{s}, a_{s+1}, \ldots, a_{k}\right)=0 \quad \forall u, \ldots, u_{s-1} .
$$

Proof. Differentiating equation (90) with respect to $u_{s}$ for $s>k$ and using the form (43), (44) of $D_{x}$ and $D_{t}$, one immediately obtains (91).

Now suppose that (92) holds for all $s>n$, where $0<n \leq k-p+1$. It easily seen that this property is preserved by any gauge transformation of the form

$$
w^{i} \mapsto f^{i}\left(w^{1}, \ldots, w^{m}, u, \ldots, u_{n-1}\right)
$$


By induction on $k-n$, it remains to find a gauge transformation (93) such that the transformed vector field $D_{x}+A$ satisfies (92) for $s=n$. Let

$$
\frac{\partial A}{\partial u_{n}}\left(u, \ldots, u_{n-1}, a_{n}, a_{n+1}, \ldots, a_{k}\right)=\sum_{j} c^{j}\left(w^{1}, \ldots, w^{m}, u, \ldots, u_{n-1}\right) \frac{\partial}{\partial w^{j}} .
$$

Similarly to the proof of Theorem 1 consider the system of ordinary differential equations

$$
\begin{gathered}
\frac{d}{d u_{n-1}} f^{j}\left(w^{1}, \ldots, w^{m}, u, \ldots, u_{n-1}\right)=c^{j}\left(f^{1}, \ldots, f^{m}, u, \ldots, u_{n-1}\right), \\
j=1, \ldots, m
\end{gathered}
$$

dependent on the parameters $w^{1}, \ldots, w^{m}$ and $u, \ldots, u_{n-2}$. Its local solution with the initial condition

$$
f^{j}\left(w^{1}, \ldots, w^{m}, u, \ldots, u_{n-2}, a_{n-1}\right)=w^{j}
$$

determines the required transformation (93).

Lemma 6. Consider two coverings

$$
\begin{gathered}
D_{x} B_{i}-D_{t} A_{i}+\left[A_{i}, B_{i}\right]=0, \\
A_{i}=\sum_{j=1}^{m_{i}} a_{i}^{j}\left(w_{i}^{1}, \ldots, w_{i}^{m_{i}}, u, \ldots, u_{k_{i}}\right) \frac{\partial}{\partial w_{i}^{j}}, \\
B_{i}=\sum_{j=1}^{m_{i}} b_{i}^{j}\left(w_{i}^{1}, \ldots, w_{i}^{m_{i}}, u, \ldots, u_{k_{i}}\right) \frac{\partial}{\partial w_{i}^{j}}, \quad i=1,2,
\end{gathered}
$$

such that both $A_{1}$ and $A_{2}$ satisfy (92) for all $s \geq 1$ and some point (89). Let

$$
w_{2}^{j}=\varphi^{j}\left(w_{1}^{1}, \ldots, w_{1}^{m_{1}}, u, u_{1}, \ldots\right), \quad j=1, \ldots, m_{2},
$$

determine a morphism of these coverings, i.e.,

$$
\begin{gathered}
\left(D_{x}+A_{1}\right) \varphi^{j}=a_{2}^{j}\left(\varphi^{1}, \ldots, \varphi^{m_{2}}, u, \ldots, u_{k_{2}}\right), \\
\left(D_{t}+B_{1}\right) \varphi^{j}=b_{2}^{j}\left(\varphi^{1}, \ldots, \varphi^{m_{2}}, u, \ldots, u_{k_{2}}\right)
\end{gathered}
$$

for all $j=1, \ldots, m$. Then functions (94) do not actually depend on any $u_{i}, i \geq 0$.

Proof. Let $r \geq 0$ be the maximal integer such that at least one of functions (94) depends nontrivially on $u_{r}$. Differentiate (95) with respect to $u_{r+1}$ and substitute $u_{i}=a_{i}$ for $i \geq r+1$. Taking into account (92) for $s=r+1$, we obtain that the right-hand side is zero, while on the left-hand side we get $\partial \varphi^{j} / \partial u_{r}$. Therefore, $\partial \varphi^{j} / \partial u_{r}=0$ for all $j$, which contradicts to our assumption.

Lemma 7. Consider covering (90) satisfying (92) for all $s \geq 1$ and let

$$
S=\sum_{j=1}^{m} s^{j}\left(w^{1}, \ldots, w^{m}, u, u_{1}, \ldots\right) \frac{\partial}{\partial w^{j}}
$$

be a symmetry of it. Then $S$ does not actually depend on any $u_{i}, i \geq 0$.

Proof. Analyzing the equation $\left[D_{x}+A, S\right]=0$ from (19), this is proved similarly to the previous lemma. 
Lemma 8. Consider covering (90) satisfying (92) for all $s \geq 1$. Let $\mathcal{E}^{\prime}$ be a subequation of $\mathcal{E} \times W$. Then locally $\mathcal{E}^{\prime}$ is of the form $\mathcal{E}_{1} \times W^{\prime}$, where $\mathcal{E}_{1}$ is an open subset of $\mathcal{E}$ and $W^{\prime}$ is a submanifold of $W$ such that all vector fields

$$
\left\{A\left(u, \ldots, u_{k}\right), B\left(u, \ldots, u_{k}\right) \in D(W) \mid u, \ldots, u_{k} \in \mathbb{C}\right\}
$$

are tangent to $W^{\prime}$.

Proof. According to Definition 3 a subequation of codimension $l$ is given by functions

$$
f_{i}\left(w^{1}, \ldots, w^{m}, u, u_{1}, \ldots\right), \quad i=1, \ldots, l,
$$

defined on an open subset $U \subset \mathcal{E} \times W$ such that

- $f_{1}(c)=\cdots=f_{l}(c)=0$ for some $c \in U$,

- the differentials

$$
d_{b} f_{i} \in T_{b}^{*}(\mathcal{E} \times W), \quad i=1, \ldots, l,
$$

are linearly independent for each $b \in U$,

- the ideal $I$ of functions on $U$ generated by $f_{1}, \ldots, f_{l}$ is preserved by the action of the vector fields $D_{x}+A, D_{t}+B$.

Let $z \in W$ be the image of $c$ under the projection $\mathcal{E} \times W \rightarrow W$. To prove the lemma, it is sufficient to find a set of functions

$$
g_{\alpha}\left(w^{1}, \ldots, w^{m}\right), \quad \alpha \in \Lambda,
$$

defined on a neighborhood of $z$ such that the ideal of functions on a neighborhood $U^{\prime} \subset U$ of $c$ generated by functions (96) coincides with $\left.I\right|_{U^{\prime}}$.

Let $r$ be the maximal integer such that at least one of the functions $f_{1}, \ldots, f_{l}$ depends nontrivially on $u_{r}$. Note that $f_{i}$ are defined on an open subset $V$ of $\mathbb{C}^{r+1} \times W$ with the coordinates $u_{0}, \ldots, u_{r}, w^{1}, \ldots, w^{m}$, the subset

$$
M=\left\{q \in V \mid f_{1}(q)=\cdots=f_{l}(q)=0\right\}
$$

is a submanifold of codimension $l$ in $V$, and $\left.I\right|_{V}$ coincides with the ideal of functions on $V$ that vanish on $M$. Thus we essentially have a question of finite-dimensional complex analysis.

Since

$$
\frac{\partial}{\partial u_{r+1}}(I) \subset I, \quad\left(D_{x}+A\right)(I) \subset I,
$$

we have

$$
\frac{\partial}{\partial u_{r+1}}\left(\left(D_{x}+A\right)\left(f_{i}\right)\right)=\frac{\partial f_{i}}{\partial u_{r}}+\frac{\partial A}{\partial u_{r+1}}\left(f_{i}\right) \in I .
$$

Substituting $u_{i}=a_{i}, i \geq r+1$, to (97), from (92) for $s=r+1$ we obtain $\partial f_{i} / \partial u_{r} \in$ $I$. Therefore, the vector field $\partial / \partial u_{r}$ is tangent to $M$, which allows to generate $I$ on some neighborhood of $c$ by functions that do not depend on $u_{i}$ for $i \geq r$. Proceeding by induction on $r$, one completes the proof.

Applying this lemma to the identity covering $\mathcal{E} \rightarrow \mathcal{E}$, we obtain the following.

Theorem 15. Any connected open subset of the translation-invariant infinite prolongation of any evolution equation (88) is irreducible.

Let us introduce some auxiliary notions. 
Definition 7. For each $i \in \mathbb{Z}_{+}$, let $V_{i}$ be a connected open subset of $\mathbb{C}$ such that for all but a finite number of $i$ we have $V_{i}=\mathbb{C}$. Set

$$
D=\left\{\left(u_{0}, u_{1}, \ldots, u_{i}, \ldots\right) \mid u_{i} \in V_{i}\right\} .
$$

Let $\mathcal{P}$ be an algebra of functions on $D$ such that each $f \in \mathcal{P}$ is a complex-analytic function dependent on a finite number of the variables $u_{i}, i \geq 0$. The algebra $\mathcal{P}$ is said to be perfect if for each function $f\left(u_{0}, \ldots, u_{r}\right) \in \mathcal{P}$ and any $i \in \mathbb{Z}_{+}$the following conditions hold.

(1) One has $\partial f / \partial u_{i} \in \mathcal{P}$.

(2) There is $g\left(u_{0}, \ldots, u_{r}\right) \in \mathcal{P}$ such that $\partial g / \partial u_{i}=f$.

(3) For any $s<r$ and any fixed numbers $a_{i} \in V_{i}, i \geq s$, we have

$$
f\left(u_{0}, \ldots, u_{s-1}, a_{s}, a_{s+1}, \ldots, a_{r}\right) \in \mathcal{P} .
$$

(4) For all $j \geq 1$ we have $u_{j} \in \mathcal{P}$.

Then each function $f \in \mathcal{P}$ is also called perfect.

Example 11. Let $V_{i}=\mathbb{C}$ and $\mathcal{P}$ be the algebra of polynomials in $u_{i}, i \geq 0$. Evidently, the algebra $\mathcal{P}$ is perfect.

Fix open subsets $V_{i} \subset \mathbb{C}$ satisfying the assumptions of Definition 7 and a perfect algebra $\mathcal{P}$.

Definition 8. Consider a vector field

$$
A=\sum_{j=1}^{m} a^{j}\left(w^{1}, \ldots, w^{m}, u, u_{1}, \ldots, u_{k}\right) \frac{\partial}{\partial w^{j}}
$$

defined on an open subset of $W \times V_{0} \times \cdots \times V_{k}$.

A vector field

$$
S=\sum_{j=1}^{m} s^{j}\left(w^{1}, \ldots, w^{m}\right) \frac{\partial}{\partial w^{j}}
$$

is said to be 1-primitive (with respect to $A$ ) if $[S, A]=0$. Now by induction on $q \in \mathbb{N}$ a vector field (99) is called q-primitive (with respect to $A$ and $\mathcal{P}$ ) if the commutator $[S, A]$ can be presented as a sum $\sum_{j=1}^{N} f_{j} S_{j}$, where $S_{j}$ are $(q-1)$-primitive fields and $f_{j}$ are perfect functions. In particular, one has $\left(\operatorname{ad}^{q} A\right)(S)=0$.

A vector field

$$
S=\sum_{j=1}^{m} s^{j}\left(w^{1}, \ldots, w^{m}, u, u_{1}, \ldots\right) \frac{\partial}{\partial w^{j}}
$$

is said to be primitive (without any prefix) if one has $S=\sum_{j=1}^{N} f_{j} S_{j}$, where $f_{j}$ are perfect functions and $S_{j}$ are $q$-primitive vector fields for some $q$.

Remark 12. Below all primitive vector fields are primitive with respect to $A$ and $\mathcal{P}$, where $\mathcal{P}$ is a fixed perfect algebra and $A$ arises from a covering (90).

Evidently, primitive vector fields form a module over the algebra $\mathcal{P}$.

Lemma 9. Consider an arbitrary vector field (100) defined on a neighborhood of the point $u_{i}=a_{i} \in V_{i}, i \geq 0$. Consider a covering (90) satisfying (92) for all $s \geq 1$. 
(1) If

$$
\frac{\partial}{\partial u_{i}}\left(D_{x}(S)+[A, S]\right)=0 \quad \forall i>0
$$

then $\partial S / \partial u_{i}=0$ for all $i \geq 0$.

(2) If $D_{x}(S)+[A, S]$ is primitive then $S$ is primitive.

(3) If $S$ is primitive and the function $F$ in (88) is perfect then $[B, S]$ is primitive.

Proof. (1) Let $r$ be the maximal integer such that $\partial S / \partial u_{r} \neq 0$. From (92) for $s=r+1$ we have

$$
\frac{\partial}{\partial u_{r+1}}\left(D_{x}(S)+[A, S]\right)\left(u, \ldots, u_{r}, a_{r+1}, \ldots, a_{k}\right)=\frac{\partial S}{\partial u_{r}} .
$$

Combining this with (101) for $i=r+1$, we obtain $\partial S / \partial u_{r}=0$.

(2) Again let $r$ be the maximal integer such that $\partial S / \partial u_{r} \neq 0$. Then (102) holds. Since $D_{x}(S)+[A, S]$ is primitive, vector field (102) is also primitive, by the properties of perfect functions. Therefore, by Condition 2 of Definition 7 there is a primitive field $S^{\prime}$ such that $\tilde{S}=S-S^{\prime}$ does not depend on $u_{i}, i \geq r$. Then $D_{x}(\tilde{S})+[A, \tilde{S}]$ is primitive, and by induction on $r$ one completes the proof.

(3) Applying ad $S$ to (90), we obtain

$$
D_{x}([S, B])+[A,[S, B]]=\left[S, D_{t} A\right]-[[S, A], B] .
$$

By assumption, for some $q$ one has

$$
S=\sum_{j} f_{j} S_{j}, \quad f_{j} \text { are perfect, } S_{j} \text { are } q \text {-primitive. }
$$

Let us prove that $[S, B]$ is primitive by induction on $q$. For $q=1$ the right-hand side of (103) is zero. Applying Part 10 this lemma to the vector field $[S, B]$, we obtain that $[S, B]$ is 1-primitive.

Now assume that the statement holds for $q-1$. Consider an arbitrary vector field $S$ satisfying (104). Let us prove that $[S, B]$ is primitive.

By formula (44), we have

$$
\left[S, D_{t} A\right]=\sum_{j=0}^{k} D_{x}^{j}(F)\left[S, \frac{\partial}{\partial u_{j}} A\right] .
$$

Since $F$ is perfect, the functions $D_{x}^{j}(F)$ are also perfect. Besides, for any primitive $X$ the vector fields $\left[X, \partial A / \partial u_{j}\right]$ are also primitive for all $j$. Therefore, (105) is primitive.

Since $[S, A]$ is a linear combination of $(q-1)$-primitive fields, the vector field $[[S, A], B]$ is also primitive by the induction assumption. Thus the right-hand side of (103) is primitive and we can apply Part 2 of this lemma to $[S, B]$.

\section{Coverings of the KdV equation}

In this section we return to the $\mathrm{KdV}$ equation

$$
u_{t}=u_{3}+u_{1} u \text {. }
$$

Our final goal here is Theorem 17 


\subsection{The canonical form of coverings.}

Theorem 16. For any covering of equation (106)

$$
\begin{gathered}
D_{x} B-D_{t} A+[A, B]=0, \\
A=A\left(u, u_{1}, \ldots, u_{k}\right), \quad B=B\left(u, u_{1}, \ldots, u_{k}\right)
\end{gathered}
$$

(we omit the dependence on fibre coordinates $w^{j}$ ) locally there is an equivalent covering such that $A, B$ are polynomial in $u_{i}$ and $A$ satisfies (92) for all $s \geq 1$ and $a_{i}=0, i \geq 1$.

Proof. Consider an arbitrary point $u_{i}=a_{i} \in \mathbb{C}, w^{j}=w_{0}^{j} \in \mathbb{C}$ where the vector fields $A$ and $B$ are defined. All local gauge transformations in this proof will be defined on a neighborhood of this point. By Lemma [5 we can assume that (92) holds for all $s \geq 1$.

Remark 13. It would be most convenient to take $a_{i}=0$ from the beginning. However, since we consider coverings over arbitrary open subsets of the translationinvariant infinite prolongation of (106), we do not know in advance whether $A, B$ are defined around this point. We will show by induction that after a suitable gauge transformation the vector fields $A, B$ become polynomial in $u_{i}$ and, therefore, are uniquely extended to the whole space of variables $u, \ldots, u_{k}$.

To clarify further arguments, let us first determine the form of $A, B$ with respect to the highest derivatives $u_{i}, i \geq k-3$. A straightforward analysis of equation (107) shows that $A$ does not depend on $u_{k}, u_{k-1}$ and is a polynomial of degree 2 in $u_{k-2}$, while $B$ is polynomial in $u_{k}, u_{k-1}, u_{k-2}$. Therefore, following the strategy of Remark 13] we can find a gauge transformation such that the transformed $A$ satisfies (92) with $a_{i}=0$ for $i \geq k-2$.

Then (92) for $s=k-2$ implies

$$
A=u_{k-2}^{2} A_{2}\left(u, \ldots, u_{k-3}\right)+A_{0}\left(u, \ldots, u_{k-3}\right) .
$$

Further analysis shows that $A_{2}$ does not depend on $u_{k-3}$ and $B$ is of the form

$$
\begin{aligned}
B=2 u_{k-2} u_{k} A_{2}-u_{k-1}^{2} A_{2}+ & B_{11}\left(u, \ldots, u_{k-3}\right) u_{k-2} u_{k-1}+ \\
& +B_{10}\left(u, \ldots, u_{k-3}\right) u_{k-1}+B_{0}\left(u, \ldots, u_{k-2}\right) .
\end{aligned}
$$

Differentiating (107) with respect to $u_{k}, u_{k-2}$, we obtain

$$
2 D_{x}\left(A_{2}\right)+B_{11}+2\left[A_{0}, A_{2}\right]=0,
$$

while differentiation with respect to $u_{k-1}, u_{k-1}$ implies

$$
-D_{x}\left(A_{2}\right)+B_{11}-\left[A_{0}, A_{2}\right]=0 .
$$

Therefore,

$$
D_{x}\left(A_{2}\right)+\left[A, A_{2}\right]=0,
$$

which by Lemma 9 (11) says that $A_{2}$ does not depend on $u_{i}, i \geq 0$, and $\left[A, A_{2}\right]=0$. That is, $A_{2}$ is 1-primitive with respect to $A$.

Definition 9. Let $r \in \mathbb{Z}_{+}$and $r<k$. A vector field

$$
A=\sum_{j=1}^{m} a^{j}\left(w^{1}, \ldots, w^{m}, u, u_{1}, \ldots, u_{k}\right) \frac{\partial}{\partial w^{j}}
$$


is said to be $r$-simple if it satisfies (92) for all $s \geq 1$ with $a_{i}=0, i \geq k-r$, and some $a_{1}, \ldots, a_{k-r-1} \in \mathbb{C}$.

Lemma 10. (1) For each $r<k$ and any covering (107) there is a locally gauge equivalent covering with $r$-simple A.

(2) If a covering (107) has $r$-simple $A$ then the vector fields

$$
\begin{aligned}
& A^{\prime}=A\left(u, \ldots, u_{k}\right)-A\left(u, \ldots, u_{k-r-1}, 0, \ldots, 0\right), \\
& B^{\prime}=B\left(u, \ldots, u_{k}\right)-B\left(u, \ldots, u_{k-r+1}, 0, \ldots, 0\right)
\end{aligned}
$$

are primitive with respect to $A$ and $\mathcal{P}$, where $\mathcal{P}$ is the perfect algebra constructed in Example 11.

Proof. For $r=2$ we proved these statements above. Suppose that the statements of hold for some $r=l \leq k-2$ and let us prove them for $r=l+1$.

By assumption, each covering is locally equivalent to a covering (107) with $l$ simple $A$. Then by Part 2 of the lemma we have

$$
\begin{aligned}
& A=A^{\prime}\left(u, \ldots, u_{k-2}\right)+\tilde{A}\left(u, \ldots, u_{k-l-1}\right), \\
& B=B^{\prime}\left(u, \ldots, u_{k}\right)+\tilde{B}\left(u, \ldots, u_{k-l+1}\right),
\end{aligned}
$$

where

$$
\tilde{A}=A\left(u, \ldots, u_{k-l-1}, 0, \ldots, 0\right), \quad \tilde{B}=B\left(u, \ldots, u_{k-l+1}, 0, \ldots, 0\right),
$$

and the primitive vector fields $A^{\prime}, B^{\prime}$ are given by (111) for $r=l$.

We can rewrite (107) as follows

$$
D_{x} \tilde{B}-D_{t} \tilde{A}+[\tilde{A}, \tilde{B}]+P=0,
$$

where

$$
P=D_{x} B^{\prime}-D_{t} A^{\prime}+\left[A, B^{\prime}\right]+\left[A^{\prime}, B\right]
$$

is primitive. Indeed, the fact that $D_{x} B^{\prime}, D_{t} A^{\prime},\left[A, B^{\prime}\right]$ are primitive follows immediately from the fact that $A^{\prime}, B^{\prime}$ are primitive, while $\left[A^{\prime}, B\right]$ is primitive by Lemma 9 (3). In particular, $P$ is polynomial in $u_{i}, i \geq 0$.

From equation (114) it follows easily that $\tilde{A}, \tilde{B}$ are polynomial in $u_{k-l-1}, u_{k-l}$, $u_{k-l+1}$. Therefore, $A\left(u, \ldots, u_{k}\right)$ and $B\left(u, \ldots, u_{k}\right)$ are defined for $u_{i}=a_{i}, i \leq$ $k-l-2$, and arbitrary values of $u_{j}, j \geq k-l-1$. By Lemma 5 after some gauge transformation

$$
w^{i} \mapsto g^{i}\left(w^{1}, \ldots, w^{m}, u, \ldots, u_{k-l-2}\right)
$$

$A$ becomes $(l+1)$-simple, which proves Part 1 of the lemma for $r=l+1$.

To prove Part 2, consider an arbitrary covering (107) with $(l+1)$-simple $A$, where $l \leq k-2$. Since $(l+1)$-simple $A$ is also $l$-simple, we again have representation (112) and equation (114), where (115) is primitive.

Similarly to formulas (108) and (109), from (114) we obtain

$$
\begin{aligned}
\tilde{A}= & P_{1}+u_{k-l-1}^{2} A_{2}^{\prime}\left(u, \ldots, u_{k-l-3}\right)+A_{0}^{\prime}\left(u, \ldots, u_{k-l-2}\right), \\
\tilde{B}=P_{2}+ & 2 u_{k-l-1} u_{k-l+1} A_{2}^{\prime}-u_{k-l}^{2} A_{2}^{\prime}+B_{11}^{\prime}\left(u, \ldots, u_{k-l-2}\right) u_{k-l-1} u_{k-l}+ \\
& +B_{10}^{\prime}\left(u, \ldots, u_{k-l-2}\right) u_{k-l}+B_{0}^{\prime}\left(u, \ldots, u_{k-l-1}\right),
\end{aligned}
$$

where $P_{1}, P_{2}$ are primitive. Similarly to (110), this implies that $D_{x} A_{2}^{\prime}+\left[A, A_{2}^{\prime}\right]$ is also primitive. By Lemma 9 (2), the vector field $A_{2}^{\prime}$ is primitive. 
Then the vector fields

$$
\begin{gathered}
A\left(u, \ldots, u_{k}\right)-A\left(u, \ldots, u_{k-l-2}, 0, \ldots, 0\right), \\
B\left(u, \ldots, u_{k}\right)-B\left(u, \ldots, u_{k-l}, 0, \ldots, 0\right)
\end{gathered}
$$

are also primitive, which proves Part 2 of the lemma for $r=l+1$.

By the above lemma for $r=k-1$, we obtain that after a suitable gauge transformation one has

$$
\begin{gathered}
A=A^{\prime \prime}\left(u, \ldots, u_{k-2}\right)+A_{0}^{\prime \prime}(u), \\
B=B^{\prime \prime}\left(u, \ldots, u_{k}\right)+B_{0}^{\prime \prime}\left(u, u_{1}, u_{2}\right),
\end{gathered}
$$

where $A^{\prime \prime}, B^{\prime \prime}$ are primitive and $A$ is $(k-1)$-simple. Now it is straightforward to prove that $A_{0}^{\prime \prime}, B_{0}^{\prime \prime}$ are polynomial in $u, u_{1}, u_{2}$.

4.2. The fundamental algebras. From the above proof it follows that for each $k \geq 3$ there are finite subsets

$$
\mathcal{M}_{k} \subset \mathbb{Z}_{+}^{k-1}, \quad \mathcal{N}_{k} \in \mathbb{Z}_{+}^{k+1}
$$

such that the following statement holds. If a covering (107) of equation (106) satisfies (92) for all $s \geq 1$ with $a_{i}=0, i \geq 1$, then it is of the form

$$
\begin{aligned}
& A=\sum_{\left(i_{0}, \ldots, i_{k-2}\right) \in \mathcal{M}_{k}} u_{0}^{i_{0}} \ldots u_{k-2}^{i_{k-2}} \mathbf{A}_{i_{0} \ldots i_{k-2}}, \\
& B=\sum_{\left(i_{0}, \ldots, i_{k}\right) \in \mathcal{N}_{k}} u_{0}^{i_{0}} \ldots u_{k}^{i_{k}} \mathbf{B}_{i_{0} \ldots i_{k}},
\end{aligned}
$$

where the vector fields

$$
\mathbf{A}_{i_{0} \ldots i_{k-2}}, \quad \mathbf{B}_{i_{0} \ldots i_{k}}
$$

do not depend on $u_{i}, i \geq 0$.

Let us show that this canonical form of coverings satisfies Definition 5 if we take

$$
\begin{gathered}
\mathcal{A}_{k}=\left\{u_{0}^{i_{0}} \ldots u_{k-2}^{i_{k-2}} \mid\left(i_{0}, \ldots, i_{k-2}\right) \in \mathcal{M}_{k}\right\}, \\
\mathcal{B}_{k}=\left\{u_{0}^{i_{0}} \ldots u_{k}^{i_{k}} \mid\left(i_{0}, \ldots, i_{k}\right) \in \mathcal{N}_{k}\right\} .
\end{gathered}
$$

Relation (64) is obvious. Condition 10 of Definition [5 follows from Theorem 16] Let

$$
S=\sum_{j=1}^{m} s^{j}\left(w^{1}, \ldots, w^{m}, u, u_{1}, \ldots\right) \frac{\partial}{\partial w^{j}}
$$

be a symmetry of the covering given by vector fields (118), i.e.,

$$
\left[D_{x}+A, S\right]=\left[D_{t}+B, S\right]=0 .
$$

By Lemma 7 does not depend on $u_{i}, i \geq 0$. Then (120) implies that $S$ commutes with all vector fields (119), which proves Condition 3 of Definition 5 . Conditions 2 and 4 follow analogously from Lemmas 6 and 8 respectively.

Vector fields (118) satisfy (107) if and only if certain Lie algebra relations hold for (119). Denote by $\mathfrak{f}_{k-2}$ the quotient of the free Lie algebra generated by letters (119) over these relations. We obtain the system of fundamental algebras

$$
\cdots \rightarrow \mathfrak{f}_{k+1} \rightarrow \mathfrak{f}_{k} \rightarrow \cdots \rightarrow \mathfrak{f}_{1} \rightarrow \mathfrak{f}_{0}
$$

for equation (106). In particular, the algebras $\mathfrak{f}_{1}$ and $\mathfrak{f}_{0}$ are described in Example 9 
Denote by $\mathfrak{a}_{k-2}$ the subalgebra of $\mathfrak{f}_{k-2}$ generated by $\mathbf{A}_{i_{0}, \ldots, i_{k-2}}$.

Lemma 11. We have

$$
\mathbf{B}_{i_{0} \ldots i_{k}} \in \mathfrak{a}_{k-2} \quad \text { for } i_{0}+\cdots+i_{k}>0 .
$$

Proof. For $\left(i_{0}, \ldots, i_{k}\right) \in \mathcal{N}_{k}$ set

$$
r\left(i_{0}, \ldots, i_{k}\right)=\max \left\{s \mid i_{s}>0\right\} .
$$

Let us prove (122) by induction on $k-r\left(i_{0}, \ldots, i_{k}\right)$.

For $\left(i_{0}, \ldots, i_{k}\right) \in \mathcal{N}_{k}$ with $r\left(i_{0}, \ldots, i_{k}\right)=k$ it follows from (109).

Suppose that (122) holds for all $\left(i_{0}, \ldots, i_{k}\right) \in \mathcal{N}_{k}$ with $r\left(i_{0}, \ldots, i_{k}\right) \geq l+1$. Differentiate (107) with respect to $u_{l+1}$ and substitute $u_{i}=0$ for $i \geq l+1$. Since $A$ satisfies (92) for $s=l+1$ and $a_{i}=0$, we obtain (122) for $\left(i_{0}, \ldots, i_{k}\right) \in \mathcal{N}_{k}$ with $r\left(i_{0}, \ldots, i_{k}\right)=l$.

Combining (122) and (107), one gets

$$
\left[\mathbf{B}_{0 \ldots 0}, \mathfrak{a}_{k-2}\right] \subset \mathfrak{a}_{k-2} .
$$

Substituting $u_{i}=0$ to (107), we obtain also

$$
\left[\mathbf{A}_{0 \ldots 0}, \mathbf{B}_{0 \ldots 0}\right]=0 \text {. }
$$

Let us specify the structure of (118). For $k=3$ it was described in Theorem 1 Similarly to the proof of Theorem 16 one obtains that for $k \geq 4$ vector fields (118) have the form

$$
\begin{gathered}
A=\mathbf{A}_{k-2}\left(u_{k-2}^{2}-\frac{2 k-3}{3} u u_{k-2}^{2}\right)+\mathbf{A}_{k-2}^{\prime} u_{k-3}^{2}+A_{0}\left(u, \ldots, u_{k-4}\right), \\
B=2 u_{k-2} u_{k} \mathbf{A}_{k-2}+B_{0}\left(u, \ldots, u_{k-1}\right),
\end{gathered}
$$

where

$$
\begin{gathered}
\mathbf{A}_{k-2}=\mathbf{A}_{0 \ldots 02}, \quad \mathbf{A}_{k-2}^{\prime}=\mathbf{A}_{0 \ldots 020}, \\
{\left[\mathbf{A}_{k-2}, A\right]=0,} \\
{\left[\mathbf{A}_{k-2}, B\right]=3\left[A_{0}, \mathbf{A}_{k-2}^{\prime}\right] .}
\end{gathered}
$$

and $A_{0}, B_{0}$ are polynomial in $u_{i}$

Equation (127) implies

$$
\left[\mathbf{A}_{k-2}, \mathfrak{a}_{k-2}\right]=0 .
$$

Combining this with (122) and (128), we obtain

$$
\left[\mathbf{A}_{k-2}, \mathbf{B}_{0 \ldots 0}\right]=3\left[\mathbf{A}_{0 \ldots 0}, \mathbf{A}_{k-2}^{\prime}\right] .
$$

Moreover, taking into account (124) and applying $\operatorname{ad}^{s} \mathbf{B}_{0 \ldots 0}$ to (130), we obtain

$$
-\left(\operatorname{ad}^{s+1} \mathbf{B}_{0 \ldots 0}\right)\left(\mathbf{A}_{k-2}\right)=3\left[\mathbf{A}_{0 \ldots 0},\left(\operatorname{ad}^{s} \mathbf{B}_{0 \ldots 0}\right)\left(\mathbf{A}_{k-2}^{\prime}\right)\right] \quad \forall s \geq 0 .
$$

By the definition of $\mathfrak{f}_{n}$ and formulas (125), (126), for each $n \geq 2$ the algebra $\mathfrak{f}_{n-1}$ is isomorphic to the quotient of $\mathfrak{f}_{n}$ over the ideal $\mathfrak{i}_{n}$ generated by $\mathbf{A}_{n}$. From (122), (123), and (131) we obtain that $\mathfrak{i}_{n} \subset \mathfrak{a}_{n}$. Moreover, (129) implies

$$
\left[\mathfrak{i}_{n}, \mathfrak{a}_{n}\right]=0 .
$$

Lemma 12. For each $n \geq 1$ we have the relation

$$
-\left(\operatorname{ad}^{n} \mathbf{B}_{0 \ldots 0}\right)\left(\mathbf{A}_{n}\right)=0
$$

in the algebra $\mathfrak{f}_{n}$. 
Proof. For $n=1$ this statement follows from (59). By induction on $n$, suppose that (133) holds for $n-1$. By formula (125), the generator $\mathbf{A}_{n}^{\prime} \in \mathfrak{f}_{n}$ is mapped to $\mathbf{A}_{n-1} \in \mathfrak{f}_{n-1}$ by the natural epimorphism $\mathfrak{f}_{n} \rightarrow \mathfrak{f}_{n} / \mathfrak{i}_{n} \cong \mathfrak{f}_{n-1}$. Therefore, $\left(\operatorname{ad}^{n-1} \mathbf{B}_{0 \ldots 0}\right)\left(\mathbf{A}_{n}^{\prime}\right) \in \mathfrak{i}_{n}$. Combining this with (131) and (132), we obtain (133).

From the above results it follows that the elements

$$
c_{i}=\left(\operatorname{ad}^{i} \mathbf{B}_{0 \ldots 0}\right)\left(\mathbf{A}_{n}\right), \quad i=0, \ldots, n-1,
$$

span the ideal $\mathfrak{i}_{n}$. The element $c_{n-1}$ belongs to the center of $\mathfrak{f}_{n}$. Moreover, for each $i=0, \ldots, n-1$ the image of $c_{i}$ belongs to the center of the quotient

$$
\mathfrak{f}_{n} /\left\langle c_{i+1}, \ldots, c_{n-1}\right\rangle \text {. }
$$

Thus we have the following statement.

Lemma 13. For each $n \geq 2$ the algebra $\mathfrak{f}_{n}$ is obtained from $\mathfrak{f}_{n-1}$ applying the operation of one-dimensional central extension no more than $n$ times.

Let us now prove the main result of this section.

Theorem 17. The $K d V$ equation (106) possesses fundamental algebras (121). Each algebra $\mathfrak{f}_{k}$ is quasi-finite and is obtained from the algebra $\mathfrak{s l}_{2}(\mathbb{C}) \otimes_{\mathbb{C}} \mathbb{C}[\lambda]$ applying several times the operation of one-dimensional central extension.

Proof. It was shown above that (121) are fundamental algebras of (106). Let us prove that algebras (121) are quasi-finite.

By Theorem 9] the algebra

$$
\mathfrak{g}=\mathfrak{s l}_{2}(\mathbb{C}) \otimes_{\mathbb{C}} \mathbb{C}[\lambda]
$$

is quasi-finite. From (59) it follows that $\mathfrak{f}_{1}$ is the trivial central extension of the algebra $\mathfrak{L}$ from Proposition 2

Since the Heisenberg algebra $H$ is nilpotent, the algebra $\mathfrak{f}_{1}$ is obtained from $\mathfrak{g}$ applying 6 times the operation of one-dimensional central extension. Therefore, by Theorem 8 , the algebra $\mathfrak{f}_{1}$ is also quasi-finite. Finally, combining Lemma 13 and Theorem 8, we obtain that all fundamental algebras (121) are quasi-finite.

It is well known that $\mathfrak{s l}_{2}(\mathbb{C}) \otimes_{\mathbb{C}} \mathbb{C}[\lambda]$ has no nontrivial central extensions. Combining this with Theorem 17 we obtain the following specification of the structure of $\mathfrak{f}_{k}$.

Theorem 18. Each algebra $\mathfrak{f}_{k}$ is isomorphic to the direct sum of $\mathfrak{s l}_{2}(\mathbb{C}) \otimes \mathbb{C}[\lambda]$ and a finite-dimensional nilpotent algebra.

\section{Coverings of the Krichever-Novikov equation}

Consider the Krichever-Novikov (KN) equation [11, 17, 18,

$$
u_{t}=u_{3}-\frac{3}{2} \frac{u_{2}^{2}}{u_{1}}+\frac{h(u)}{u_{1}}, \quad u_{k}=\frac{\partial^{k} u}{\partial x^{k}}
$$

where $h(u)$ is a polynomial of degree 3 with coefficients in $\mathbb{C}$. If the roots of the polynomial $h(u)$ are distinct then equation (134) is said to be nonsingular.

The main goal of this section is Theorem 22 
5.1. The canonical form of coverings. We want to have an analog of Theorem [16 for equation (134). The straightforward repetition of the proof of Theorem 16] is not possible, because (134) is not polynomial in $u_{1}$.

To overcome this, we need to introduce a perfect algebra that contains the function $1 / u_{1}$. By Condition 2 of Definition 7 this algebra must contain also $\int 1 / u_{1} d u_{1}$.

To this end, choose a half-line $L \subset \mathbb{C}$ from 0 to $\infty$ such that $V_{1}=\mathbb{C} \backslash L$ is simply connected. Let $\ln u_{1}$ be a single-valued branch of the logarithm defined on $V_{1}$. Set $V_{i}=\mathbb{C}, i \neq 1$, and let $\mathcal{P}$ be the algebra of polynomials in

$$
u_{i}, i \geq 0, \quad \frac{1}{u_{1}}, \quad \ln u_{1} \text {. }
$$

Then $\mathcal{P}$ is a perfect algebra. Indeed, all conditions of Definition 7 are obvious except of Condition 2 The latter follows from the fact that for any $a \in \mathbb{Z}, b \in \mathbb{Z}_{+}$ there is a polynomial $g$ in $u_{1}, 1 / u_{1}, \ln u_{1}$ such that $\partial g / \partial u_{1}=u_{1}^{a} \ln ^{b} u_{1}$.

Remark 14. Thus for equation (134) we study not the whole translation-invariant infinite prolongation, but the open dense subset

$$
\left\{\left(u_{0}, u_{1}, \ldots\right) \mid u_{1} \in \mathbb{C} \backslash L, \quad u_{i} \in \mathbb{C} \quad \forall i \neq 1\right\}
$$

of it.

In Theorem [16] we proved that every covering of the KdV equation is locally equivalent to a covering in the canonical form satisfying (92) for all $s \geq 1$ and $a_{i}=0, i \geq 1$. For equation (134) the point $u_{i}=0$ is also crucial. However, one cannot prove the same statement for coverings of (134), because $1 / u_{1}$ and $\ln u_{1}$ are not defined at $u_{1}=0$. Let us make necessary modifications.

Definition 10. A vector field

$$
S=\sum_{j=1}^{m} s^{j}\left(w^{1}, \ldots, w^{m}, u, \ldots, u_{k}\right) \frac{\partial}{\partial w^{j}}
$$

is said to be $u_{1}$-free if each function $s^{j}\left(w^{1}, \ldots, w^{m}, u, \ldots, u_{k}\right)$ is polynomial in

$$
u_{i}, i \geq 1, \quad \frac{1}{u_{1}}, \quad \ln u_{1}
$$

with coefficients dependent on $u, w^{1}, \ldots, w^{m}$ and the coefficient at the monomial $u_{1}$ is zero. (This coefficient is well defined because the functions

$$
u_{1}^{a} \ln ^{b} u_{1}, \quad a \in \mathbb{Z}, \quad b \in \mathbb{Z}_{+},
$$

are linearly independent.)

Definition 11. Let $r \in \mathbb{Z}_{+}$and $r \leq k-2$. A vector field

$$
A=\sum_{j=1}^{m} a^{j}\left(w^{1}, \ldots, w^{m}, u, u_{1}, \ldots, u_{k}\right) \frac{\partial}{\partial w^{j}}
$$

is said to be weakly $r$-simple if it satisfies (92) for all $s \geq 2$ with $a_{i}=0, i \geq k-r$, and some $a_{2}, \ldots, a_{k-r-1} \in \mathbb{C}$.

In contrast to $r$-simple vector fields, a weakly $r$-simple vector field does not necessarily satisfy (92) for $s=1$.

Remark 15. In this section perfect functions are elements of the perfect algebra $\mathcal{P}$ defined above. 
Lemma 14. If in Lemmas [6, 7, 8, , one replaces the condition that A satisfies (92) for all $s \geq 1$ by the condition that $A$ is $u_{1}$-free and weakly $(k-2)$-simple then the conclusions of these lemmas remain valid.

Proof. Let us prove that Lemma 9(1) remains valid, since the other statements are proved analogously.

So assume that $A\left(u, \ldots, u_{k}\right)$ is $u_{1}$-free and weakly $(k-2)$-simple and that equation (101) holds. By Definition [1] $A$ satisfies (92) for all $s \geq 2$. Therefore, the equations

$$
\frac{\partial S}{\partial u_{i}}=0 \quad \forall i \geq 1
$$

are proved in the same way as in Lemma 9 (11).

Let us prove that $\partial S / \partial u$ is also equal to zero. From (101) for $i=1$ we have

$$
\frac{\partial S(u)}{\partial u}+\left[\frac{\partial A}{\partial u_{1}}, S(u)\right]=0 .
$$

Since $A$ is $u_{1}$-free and (138) holds, the vector field $\left[\partial A / \partial u_{1}, S(u)\right]$ is either zero or depends nontrivially on some $u_{i}, i \geq 1$. Combining this with (138) and (139), we obtain

$$
\frac{\partial S}{\partial u}=\left[\frac{\partial A}{\partial u_{1}}, S(u)\right]=0 .
$$

Theorem 19. For any covering of equation 134

$$
\begin{gathered}
D_{x} B-D_{t} A+[A, B]=0, \\
A=A\left(u, u_{1}, \ldots, u_{k}\right), \quad B=B\left(u, u_{1}, \ldots, u_{k}\right)
\end{gathered}
$$

(we omit the dependence on fibre coordinates $w^{j}$ ) locally there is an equivalent covering such that

(1) $A, B$ are polynomial in (135),

(2) $A$ is $(k-2)$-simple and $u_{1}$-free.

Proof. Let (141) be defined on a neighborhood of a point $u_{i}=a_{i}$.

Lemma 15. (1) For each $r \leq k-2$ and any covering (140) there is a locally gauge equivalent covering with $r$-simple $A$.

(2) If a covering (140) has $r$-simple $A$ then the vector fields

$$
\begin{aligned}
& A^{\prime}=A\left(u, \ldots, u_{k}\right)-A\left(u, \ldots, u_{k-r-1}, 0, \ldots, 0\right), \\
& B^{\prime}=B\left(u, \ldots, u_{k}\right)-B\left(u, \ldots, u_{k-r+1}, 0, \ldots, 0\right)
\end{aligned}
$$

are primitive with respect to $A$ and $\mathcal{P}$.

Proof. This is proved similarly to Lemma 10 Formulas (116) and (117) for $l \leq k-3$ are replaced by

$$
\begin{gathered}
\tilde{A}=P_{1}+\frac{u_{k-l-1}^{2}}{u_{1}^{2}} A_{2}^{\prime}+A_{0}^{\prime}\left(u, \ldots, u_{k-l-2}\right), \\
\tilde{B}=P_{2}+2 \frac{u_{k-l-1} u_{k-l+1}}{u_{1}^{2}} A_{2}^{\prime}-\frac{u_{k-l}^{2}}{u_{1}^{2}} A_{2}^{\prime}+B_{11}^{\prime}\left(u, \ldots, u_{k-l-2}\right) u_{k-l-1} u_{k-l}+ \\
+B_{10}^{\prime}\left(u, \ldots, u_{k-l-2}\right) u_{k-l}+B_{0}^{\prime}\left(u, \ldots, u_{k-l-1}\right),
\end{gathered}
$$

where $P_{1}, P_{2}, A_{2}^{\prime}$ are primitive. 
By the above lemma for $r=k-2$, after a suitable gauge transformation we have

$$
\begin{aligned}
& A=A^{\prime}+A_{0}\left(u, u_{1}\right), \\
& B=B^{\prime}+B_{0}\left(u, u_{1}, u_{2}, u_{3}\right),
\end{aligned}
$$

where the vector fields

$$
\begin{aligned}
& A^{\prime}=A\left(u, \ldots, u_{k}\right)-A\left(u, u_{1}, 0, \ldots, 0\right), \\
& B^{\prime}=B\left(u, \ldots, u_{k}\right)-B\left(u, u_{2}, u_{3}, 0, \ldots, 0\right)
\end{aligned}
$$

are primitive and $A$ is $(k-2)$-simple.

Substituting (142) to (140), it is straightforward to obtain that

$$
A_{0}=C+\frac{1}{u_{1}} A_{1}(u)+u_{1} A_{2}(u)+A_{3}(u),
$$

where $C$ is primitive.

The vector field $A$ remains weakly ( $k-2)$-simple and polynomial in (137) after any gauge transformation of the form

$$
w^{i} \mapsto f^{i}\left(w^{1}, \ldots, w^{m}, u\right) .
$$

Let us find a gauge transformation (145) such that $A$ becomes $u_{1}$-free. To this end, let

$$
\sum_{j=1}^{m} c^{j}\left(w^{1}, \ldots, w^{m}, u\right) \frac{\partial}{\partial w^{j}}
$$

be the coefficient of $A$ at the monomial $u_{1}$ and consider the system of ordinary differential equations

$$
\frac{d}{d u} f^{j}\left(w^{1}, \ldots, w^{m}, u\right)=c^{j}\left(f^{1}, \ldots, f^{m}, u\right), \quad j=1, \ldots, m,
$$

dependent on the parameters $w^{1}, \ldots, w^{m}$. Its local solution with the initial condition

$$
f^{j}\left(w^{1}, \ldots, w^{m}, a_{0}\right)=w^{j}, \quad j=1, \ldots, m,
$$

determines gauge transformation (145) that makes $A u_{1}$-free.

By Lemma 14, in Lemma 15]2) for $r=k-2$ the condition that $A$ is $(k-2)$ simple can be replaced by the condition that $A$ is weakly $(k-2)$-simple and $u_{1}$-free. Therefore, after this gauge transformation vector fields (143) remain primitive and we have formula (144) with primitive $u_{1}$-free $C$ and $A_{2}(u)=0$.

Now it is straightforward to show that

$$
A\left(u, u_{1}, 0, \ldots, 0\right), \quad B\left(u, u_{2}, u_{3}, 0, \ldots, 0\right)
$$

are polynomial in (135). Therefore, $A$ and $B$ satisfy the conditions of the theorem.

5.2. The fundamental algebras. Consider the following set of perfect functions

$$
\begin{aligned}
Z=\left\{\left(\ln ^{a} u_{1}\right) u_{0}^{i_{0}} u_{1}^{i_{1}} u_{2}^{i_{2}} \ldots u_{k}^{i_{k}} \mid i_{1} \in \mathbb{Z},\right. \\
\\
\left.a, i_{0}, i_{2}, \ldots, i_{k} \in \mathbb{Z}_{+},\left|i_{1}\right|+a+i_{0}+i_{2}+\cdots+i_{k}>0\right\} .
\end{aligned}
$$

Similarly to the case of the KdV equation, from the proof of Theorem 19 it follows that for each $k \geq 3$ there are finite subsets

$$
\mathcal{A}_{k}^{\prime}, \mathcal{B}_{k}^{\prime} \subset Z, \quad \mathcal{A}_{k}^{\prime} \subset \mathcal{A}_{k+1}^{\prime}, \quad \mathcal{B}_{k}^{\prime} \subset \mathcal{B}_{k+1}^{\prime}
$$


such that the following statement holds. If a covering (140) of equation (134) has $(k-2)$-simple $u_{1}$-free $A$ then it is of the form

$$
A=\sum_{f \in \mathcal{A}_{k}^{\prime}} f \mathbf{A}_{f}+\mathbf{A}_{1}, \quad B=\sum_{g \in \mathcal{B}_{k}^{\prime}} g \mathbf{B}_{g}+\mathbf{B}_{1},
$$

where the vector fields

$$
\mathbf{A}_{f}, \mathbf{B}_{g}, \mathbf{A}_{1}, \mathbf{B}_{1}
$$

do not depend on $u_{i}, i \geq 0$.

Let us show that the conditions of Definition 5 hold, if we set

$$
\mathcal{A}_{k}=\mathcal{A}_{k}^{\prime} \cup\{1\}, \quad \mathcal{B}_{k}=\mathcal{B}_{k}^{\prime} \cup\{1\} .
$$

Indeed, Condition 1 follows from Theorem 19] Conditions 2 , 3 , 4 hold because, by Lemma 14 Lemmas 6 17 8 are applicable to the canonical form of coverings described in Theorem 19]

Vector fields (147) satisfy (140) if and only if certain Lie algebra relations hold for (119). Denote by $\mathfrak{f}_{k-2}^{K N}$ the quotient of the free Lie algebra generated by letters (119) over these relations. We obtain the system of fundamental algebras

$$
\cdots \rightarrow \mathfrak{f}_{n+1}^{K N} \rightarrow \mathfrak{f}_{n}^{K N} \rightarrow \cdots \rightarrow \mathfrak{f}_{1}^{K N} \rightarrow \mathfrak{f}_{0}^{K N}
$$

for equation (134).

Proposition $4(17)$. For each integer $n \geq 2$ there is a conserved current $D_{t} f_{n}=$ $D_{x} g_{n}$ of the form

$$
f_{n}=\frac{u_{n}^{2}}{u_{1}^{2}}+\tilde{f}_{n}\left(u, \ldots, u_{n-1}\right), \quad g_{n}=2 \frac{u_{n} u_{n+2}}{u_{1}^{2}}+\tilde{g}_{n}\left(u, \ldots, u_{n+1}\right),
$$

where $\tilde{f}_{n}, \tilde{g}_{n}$ are polynomials in $1 / u_{1}, u_{i}, i \geq 0$.

Similarly to Lemma 5 we can find equivalent conserved currents

$$
f_{n}^{\prime}=f_{n}+D_{x}\left(h_{n}\left(u, \ldots, u_{n-2}\right)\right), \quad g_{n}^{\prime}=g_{n}+D_{t}\left(h_{n}\left(u, \ldots, u_{n-2}\right)\right)
$$

such that

- the functions $f_{n}^{\prime}, g_{n}^{\prime}$ are perfect,

- we have

$$
\frac{\partial f_{n}^{\prime}}{\partial u_{s}}\left(u, \ldots, u_{s-1}, 0, \ldots, 0\right)=0 \quad \forall s \geq 2,
$$

- $f_{n}^{\prime}$ is polynomial in (137) with zero coefficient at the monomial $u_{1}$.

Example 12. We have

$$
\begin{gathered}
f_{2}^{\prime}=\frac{u_{2}^{2}}{u_{1}^{2}}+\frac{2}{3} \frac{h(u)}{u_{1}^{2}}, \\
g_{2}^{\prime}=2 \frac{u_{2} u_{4}}{u_{1}^{2}}-\frac{u_{3}^{2}}{u_{1}^{2}}-\frac{4}{3} \frac{h(u) u_{3}}{u_{1}^{3}}-4 \frac{u_{2}^{2} u_{3}}{u_{1}^{3}}+ \\
+\frac{9}{4} \frac{u_{2}^{4}}{u_{1}^{4}}-h(u) \frac{u_{2}^{2}}{u_{1}^{4}}+2 \frac{d h(u)}{d u} \frac{u_{2}}{u_{1}^{2}}-\frac{1}{3} \frac{h(u)^{2}}{u_{1}^{4}} .
\end{gathered}
$$


Return to algebras (149). Let $\mathfrak{a}_{k} \subset \mathfrak{f}_{k-2}^{K N}$ be the subalgebra generated by $\mathbf{A}_{f}, f \in$ $\mathcal{A}_{k}^{\prime}$, and $\tilde{\mathfrak{a}}_{k} \subset \mathfrak{f}_{k-2}^{K N}$ be the subalgebra generated by $\mathfrak{a}_{k-2}$ and $\mathbf{A}_{1}$. Similarly to Lemma 11] we obtain

$$
\begin{gathered}
\mathbf{B}_{g} \in \mathfrak{a}_{k-2} \quad \forall g \in \mathcal{B}_{k}^{\prime}, \\
{\left[\mathbf{B}_{1}, \tilde{\mathfrak{a}}_{k-2}\right] \subset \mathfrak{a}_{k-2} .}
\end{gathered}
$$

For $k \geq 5$ vector fields (147) can be rewritten as follows

$$
\begin{gathered}
A=f_{k-2}^{\prime} \mathbf{A}^{k-2}+\frac{u_{k-3}^{2}}{u_{1}^{2}} \tilde{\mathbf{A}}^{k-2}+A_{0}\left(u, \ldots, u_{k-4}\right), \\
B=g_{k-2}^{\prime} \mathbf{A}^{k-2}+B_{0}\left(u, \ldots, u_{k-1}\right),
\end{gathered}
$$

where

$$
\begin{gathered}
\mathbf{A}^{k-2}=\mathbf{A}_{u_{k-2}^{2} u_{1}^{-2}}, \quad \tilde{\mathbf{A}}^{k-2}=\mathbf{A}_{u_{k-3}^{2} u_{1}^{-2}}, \\
{\left[\mathbf{A}^{k-2}, A\right]=0,} \\
{\left[\mathbf{A}^{k-2}, B\right]=3\left[A_{0}, \tilde{\mathbf{A}}^{k-2}\right] .}
\end{gathered}
$$

Equation (154) implies

$$
\left[\mathbf{A}^{k-2}, \tilde{\mathfrak{a}}_{k-2}\right]=0 .
$$

Combining this with (150) and (155), we obtain

$$
\begin{gathered}
{\left[\mathbf{A}^{k-2}, \mathbf{B}_{1}\right]=3\left[\mathbf{A}_{1}, \tilde{\mathbf{A}}^{k-2}\right],} \\
{\left[\tilde{\mathbf{A}}^{k-2}, \mathfrak{a}_{k-2}\right]=0 .}
\end{gathered}
$$

Taking into account (157), (151) and applying ad ${ }^{s} \mathbf{B}_{1}$ to (156), we obtain

$$
-\left(\operatorname{ad}^{s+1} \mathbf{B}_{1}\right)\left(\mathbf{A}^{k-2}\right)=3\left[\mathbf{A}_{1},\left(\operatorname{ad}^{s} \mathbf{B}_{1}\right)\left(\tilde{\mathbf{A}}^{k-2}\right)\right] \quad \forall s \geq 0 .
$$

Similarly to Section 4.2 the obtained identities imply that for each $k \geq 5$ the algebra $\mathfrak{f}_{k-2}^{K N}$ is obtained from $\mathfrak{f}_{k-3}^{K N}$ applying several times the operation of one-dimensional central extension.

Let us describe the algebras $\mathfrak{f}_{i}^{K N}$ for $i=0,1,2$.

Theorem 20. Any covering of equation (134) of the form

$$
\begin{gathered}
D_{x} B-D_{t} A+[A, B]=0, \\
A=A\left(u, u_{1}, u_{2}, u_{3}, u_{4}\right), \quad B=B\left(u, u_{1}, u_{2}, u_{3}, u_{4}\right)
\end{gathered}
$$

is locally equivalent to a covering of the form

$$
\begin{gathered}
A=f_{2}^{\prime} C+\frac{1}{u_{1}} A_{1}(u)+V_{1}, \\
B=g_{2}^{\prime} C-\frac{u_{3}}{u_{1}^{2}} A_{1}+\frac{u_{2}^{2}}{2 u_{1}^{3}} A_{1}+\frac{2 u_{2}}{u_{1}} \frac{\partial A_{1}}{\partial u}-\frac{h(u)}{3 u_{1}^{3}} A_{1}+ \\
+\frac{2}{u_{1}}\left[A_{1}, \frac{\partial A_{1}}{\partial u}\right]-2 u_{1} \frac{\partial^{2} A_{1}}{\partial u^{2}}+V_{2},
\end{gathered}
$$

where $A_{1}=A_{10}+u A_{11}+u^{2} A_{12}$, the vector fields $C, V_{i}, A_{1 k}$ do not depend on $u_{i}, i \geq 0$, and are subject to the following relations

$$
\begin{gathered}
{\left[C, V_{i}\right]=\left[C, A_{1 k}\right]=\left[V_{1}, V_{2}\right]=\left[V_{i}, A_{1 k}\right]=0 \quad i=1,2, \quad k=0,1,2,} \\
2 h(u) \frac{\partial A_{1}}{\partial u}-\frac{d h(u)}{d u} A_{1}-3\left[A_{1},\left[A_{1}, \frac{\partial A_{1}}{\partial u}\right]\right]=0 .
\end{gathered}
$$


Proof. This is proved by a straightforward computation following the scheme of the proof of Theorem [19] Relation (160) was obtained in [6].

Equation (160) determines some relations between the vector fields $A_{1 k}, k=$ $0,1,2$. Let us describe the quotient of the free Lie algebra generated by $A_{1 k}$ over these relations.

Consider the ideal $\mathcal{I} \subset \mathbb{C}\left[v_{1}, v_{2}, v_{3}\right]$ generated by the polynomials

$$
v_{i}^{2}-v_{j}^{2}+\frac{8}{3}\left(e_{j}-e_{i}\right), \quad i, j=1,2,3,
$$

where $e_{1}, e_{2}, e_{3}$ are the roots of the polynomial $h(u)$. Set

$$
E=\mathbb{C}\left[v_{1}, v_{2}, v_{3}\right] / \mathcal{I} \text {. }
$$

That is, $E$ is the ring of regular functions on the affine elliptic curve in $\mathbb{C}^{3}$ defined by polynomials (161). The image of $v_{j} \in \mathbb{C}\left[v_{1}, v_{2}, v_{3}\right]$ in $E$ is denoted by $\bar{v}_{j}$. Consider also a basis $x_{1}, x_{2}, x_{3}$ of the Lie algebra $\mathfrak{s l}_{2}(\mathbb{C}) \cong \mathfrak{s o}_{3}(\mathbb{C})$ with the relations

$$
\left[x_{1}, x_{2}\right]=x_{3}, \quad\left[x_{2}, x_{3}\right]=x_{1}, \quad\left[x_{3}, x_{1}\right]=x_{2}
$$

and endow the space $L=\mathfrak{s l}_{2} \otimes_{\mathbb{C}} E$ with the Lie algebra structure described in (76).

Proposition 5 (6]). Suppose that the roots $e_{1}, e_{2}, e_{3}$ of $h(u)$ are distinct. The quotient of the free Lie algebra generated by $A_{1 k}, k=0,1,2$, over relations (160) is isomorphic to the subalgebra $\mathfrak{R} \subset L$ generated by the elements

$$
x_{1} \otimes \bar{v}_{1}, x_{2} \otimes \bar{v}_{2}, x_{3} \otimes \bar{v}_{3} \in L .
$$

From (159) we obtain

$$
\mathfrak{f}_{0}^{K N}=0, \quad \mathfrak{f}_{1}^{K N} \cong \mathfrak{R} \oplus \mathbb{C}^{2}, \quad \mathfrak{f}_{2}^{K N} \cong \mathfrak{R} \oplus \mathbb{C}^{3} .
$$

Theorem 21. The algebra $\mathfrak{R}$ is quasi-finite.

Proof. Below we assume everywhere that $\{j, k, l\}=\{1,2,3\}$. For each $j=1,2,3$ consider the subspace $V_{j} \subset \mathbb{C}\left[v_{1}, v_{2}, v_{3}\right]$ spanned by the monomials $v_{j}^{d_{j}} v_{k}^{d_{k}} v_{l}^{d_{l}}$ satisfying

$$
d_{j} \equiv d_{k}+1 \equiv d_{l}+1 \quad \bmod 2 .
$$

Denote by $R_{j}$ the image of $V_{j}$ in the quotient space $E$.

The algebra $\mathfrak{R}$ was also studied in [14 in connection with coverings of the Landau-Lifshitz equation. In the proof of Lemma 3.1 of [14] it is shown that $\mathfrak{R}=\oplus_{j=1}^{3}\left\langle x_{j}\right\rangle \otimes R_{j}$.

Let $\mathfrak{h} \subset \mathfrak{R}$ be a subalgebra of finite codimension. Then the subspace $H_{j}=\{f \in$ $\left.R_{j} \mid x_{j} \otimes f \in \mathfrak{h}\right\}$ is of finite codimension in $R_{j}$ for each $j=1,2,3$. In addition, from the definition of $R_{j}$ and relations (162) we have

$$
R_{j} R_{k} \subset R_{l}, \quad H_{j} H_{k} \subset H_{l} .
$$

This implies that for all $j=1,2,3$ the subspace

$$
H_{j}^{\prime}=\left\{a \in H_{j} \mid a R_{k} \subset H_{l}, a R_{l} \subset H_{k}\right\}
$$

is also of finite codimension in $R_{j}$. From (164) and (165) one gets

$$
H_{j}^{\prime} H_{k}^{\prime} \subset H_{l}^{\prime}, \quad H_{j}^{\prime} R_{j} R_{k} R_{l} \subset H_{j}^{\prime} \text {. }
$$

It is easy to see that $R_{j}=\left\langle\bar{v}_{j}\right\rangle+R_{k} R_{l}$. Therefore,

$$
R_{j}^{2}=\left\langle\bar{v}_{j}^{2}\right\rangle+R_{j} R_{k} R_{l}
$$


For each $j=1,2,3$ the subspace

$$
H_{j}^{\prime \prime}=\left\{a \in H_{j}^{\prime} \mid a \bar{v}_{k}^{2} \subset H_{j}^{\prime}, a \bar{v}_{l}^{2} \subset H_{j}^{\prime}, a R_{k} \subset H_{l}^{\prime}, a R_{l} \subset H_{k}^{\prime}\right\}
$$

is of finite codimension in $H_{j}^{\prime}$ and, therefore, in $R_{j}$. By definitions (168), (165) and properties (164), (166), (167), one gets

$$
R_{k} H_{j}^{\prime \prime} \subset H_{l}^{\prime \prime}, \quad R_{l} H_{j}^{\prime \prime} \subset H_{k}^{\prime \prime},
$$

which implies that $\oplus_{j=1}^{3}\left\langle x_{j}\right\rangle \otimes H_{j}^{\prime \prime} \subset \mathfrak{h}$ is an ideal of $\mathfrak{R}$. Since $H_{j}^{\prime \prime}$ is of finite codimension in $R_{j}$, this ideal is of finite codimension in $\mathfrak{R}$.

Collecting the results of this subsection and taking into account Theorems 21 and 8 one obtains the following.

Theorem 22. The nonsingular Krichever-Novikov equation (134) possesses fundamental algebras (149), where $\mathfrak{f}_{0}^{K N}=0$. Each $\mathfrak{f}_{n}^{K N}$ for $n>0$ is quasi-finite and is obtained from $\mathfrak{R}$ applying several times the operation of one-dimensional central extension.

\section{Coverings of the equation $u_{t}=u_{x x x}$}

In this section we study the linear equation

$$
u_{t}=u_{x x x} \text {. }
$$

The following theorem is proved by a straightforward computation.

Theorem 23. Any Wahlquist-Estabrook covering

$$
\begin{gathered}
D_{x} B-D_{t} A+[A, B]=0, \\
A=A\left(u, u_{1}, u_{2}\right), \quad B=B\left(u, u_{1}, u_{2}\right)
\end{gathered}
$$

of equation (169) is of the form

$$
\begin{gathered}
A=u^{2} A_{2}+u A_{1}+A_{0} \\
B=u_{2}\left(2 u A_{2}+A_{1}\right)-u_{1}^{2} A_{2}+u_{1}\left[A_{1}, A_{0}\right]-\frac{1}{2} u^{2}\left[A_{1},\left[A_{1}, A_{0}\right]\right]+ \\
+u\left[A_{0},\left[A_{0}, A_{1}\right]\right]+B_{0},
\end{gathered}
$$

where the vector fields $A_{i}, B_{0}$ depend only on $w^{1}, \ldots, w^{m}$ and are subject to the relations

$$
\begin{gathered}
{\left[A_{0}, A_{2}\right]=\left[A_{1}, A_{2}\right]=0,} \\
{\left[A_{0}, B_{0}\right]=0,} \\
{\left[A_{1},\left[A_{1},\left[A_{1}, A_{0}\right]\right]\right]=0,} \\
{\left[A_{2}, B_{0}\right]=\frac{3}{2}\left[A_{0},\left[A_{1},\left[A_{1}, A_{0}\right]\right]\right],} \\
{\left[B_{0}, A_{1}\right]=\left[A_{0},\left[A_{0},\left[A_{0}, A_{1}\right]\right]\right] .}
\end{gathered}
$$

Denote by $\mathfrak{N}$ the quotient of the free Lie algebra generated by $A_{i}, B_{0}$ over relations (170), 1171), 172), 1733, 174). Similarly to Section 4.1 one proves the following.

Theorem 24. Equation (169) possesses a system of fundamental algebras, which are obtained from $\mathfrak{N}$ applying several times the operation of one-dimensional central extension. 
Let us present some information on the structure of $\mathfrak{N}$.

Theorem 25. There are ideals $\mathfrak{N}_{i}, i \in \mathbb{Z}_{+}$, of $\mathfrak{N}$ such that

- $\mathfrak{N}_{0}=0, \quad \mathfrak{N}_{i} \subset \mathfrak{N}_{i+1} \quad \forall i \in \mathbb{Z}_{+}$,

- the quotient $\mathfrak{N}_{i+1} / \mathfrak{N}_{i}$ is commutative for all $i \in \mathbb{Z}_{+}$,

- the quotient $\mathfrak{N} / \cup_{i} \mathfrak{N}_{i}$ is solvable.

Proof. For a subset $S$ of a Lie algebra we denote by $\langle S\rangle$ the ideal generated by this subset. For simplicity, below the images of $A_{i}, B_{0} \in \mathfrak{N}$ in quotients of $\mathfrak{N}$ are denoted by the same symbols $A_{i}, B_{0}$. From the relations that define the algebra $\mathfrak{N}$ one easily obtains the following.

Lemma 16. Let $Q$ be a quotient algebra of $\mathfrak{N}$ and $C$ be an element of the subalgebra of $Q$ generated by $A_{i}$. If $\left[A_{0}, C\right]=\left[A_{1}, C\right]=0$ then the ideal $\langle C\rangle \subset Q$ is spanned by the elements $\left(\mathrm{ad}^{k} B_{0}\right)(C), k \in \mathbb{Z}_{+}$, and is commutative.

Let us construct the required ideals $\mathfrak{N}_{i}$. Set $\mathfrak{N}_{0}=0$ and $\mathfrak{N}_{1}=\left\langle A_{2}\right\rangle$. Combining relation (170) with the above lemma, we see that $\mathfrak{N}_{1} / \mathfrak{N}_{0}=\mathfrak{N}_{1}$ is commutative. By induction on $i \in \mathbb{N}$, set

$$
\mathfrak{N}_{i+1}=\left\langle\mathfrak{N}_{i},\left[A_{1},\left(\operatorname{ad}^{2 i-1} A_{0}\right)\left(A_{1}\right)\right]\right\rangle .
$$

Lemma 17. For all $i \geq 1$ in the quotient algebra $\mathfrak{N} / \mathfrak{N}_{i}$ we have

$$
\begin{gathered}
{\left[\left(\operatorname{ad}^{k} A_{0}\right)\left(A_{1}\right),\left(\operatorname{ad}^{l} A_{0}\right)\left(A_{1}\right)\right]=0 \quad \forall k, l \in \mathbb{Z}_{+} \quad k+l \leq 2 i-2,} \\
{\left[A_{0},\left[A_{1},\left(\operatorname{ad}^{2 i-1} A_{0}\right)\left(A_{1}\right)\right]\right]=\left[A_{1},\left[A_{1},\left(\operatorname{ad}^{2 i-1} A_{0}\right)\left(A_{1}\right)\right]\right]=0 .}
\end{gathered}
$$

Proof. Let us prove this by induction on $i$. For $i=1$ relation (176) is trivial, and relation (177) follows from (172) and (173). Suppose that the statement holds for $i=n \geq 1$ and let us prove it for $i=n+1$. By the induction assumption, relations (176) for $i=n$ hold in $\mathfrak{N} / \mathfrak{N}_{n+1}$. By definition (175), we have also

$$
\left[A_{1},\left(\operatorname{ad}^{2 n-1} A_{0}\right)\left(A_{1}\right)\right]=0 .
$$

Applying the Jacobi identity to (178) and taking into account (176) for $i=n$, we obtain

$$
\left[\left(\operatorname{ad}^{k} A_{0}\right)\left(A_{1}\right),\left(\operatorname{ad}^{l} A_{0}\right)\left(A_{1}\right)\right]=0 \quad \forall k, l \in \mathbb{Z}_{+} k+l \leq 2 n-1 .
$$

By the same argument, we have

$$
\begin{aligned}
& {\left[\left(\operatorname{ad}^{k} A_{0}\right)\left(A_{1}\right),\left(\operatorname{ad}^{l} A_{0}\right)\left(A_{1}\right)\right]=} \\
& \quad=-\left[\left(\operatorname{ad}^{k+1} A_{0}\right)\left(A_{1}\right),\left(\operatorname{ad}^{l-1} A_{0}\right)\left(A_{1}\right)\right] \quad \forall k+l=2 n .
\end{aligned}
$$

Using this, we obtain

$$
\begin{aligned}
& {\left[\left(\operatorname{ad}^{k} A_{0}\right)\left(A_{1}\right),\left(\operatorname{ad}^{l} A_{0}\right)\right.} \\
& \left.\quad\left(A_{1}\right)\right]= \\
& =\left[\left(\operatorname{ad}^{l} A_{0}\right)\left(A_{1}\right),\left(\operatorname{ad}^{k} A_{0}\right)\left(A_{1}\right)\right]=0 \quad \forall k+l=2 n .
\end{aligned}
$$

Relations (179) and (181) imply (176) for $i=n+1$.

It remains to prove (177) for $i=n+1$, that is,

$$
\begin{aligned}
& {\left[A_{0},\left[A_{1},\left(\operatorname{ad}^{2 n+1} A_{0}\right)\left(A_{1}\right)\right]\right]=0,} \\
& {\left[A_{1},\left[A_{1},\left(\operatorname{ad}^{2 n+1} A_{0}\right)\left(A_{1}\right)\right]\right]=0 .}
\end{aligned}
$$

Relation (183) follows easily from the Jacobi identity combined with (176) for $i=$ $n+1$. 
Similarly to (180) we have

$$
\begin{aligned}
& {\left[\left(\operatorname{ad}^{k} A_{0}\right)\left(A_{1}\right),\left(\operatorname{ad}^{l} A_{0}\right)\left(A_{1}\right)\right]=} \\
& \quad=-\left[\left(\operatorname{ad}^{k+1} A_{0}\right)\left(A_{1}\right),\left(\operatorname{ad}^{l-1} A_{0}\right)\left(A_{1}\right)\right] \quad \forall k+l=2 n+1 .
\end{aligned}
$$

Set $\left.I=\left[A_{1},\left(\operatorname{ad}^{2 n+1} A_{0}\right)\left(A_{1}\right)\right]\right]$. Using (184), one gets

$$
\begin{aligned}
I=(-1)^{n}\left[\left(\operatorname{ad}^{n} A_{0}\right)\left(A_{1}\right),\left(\operatorname{ad}^{n+1} A_{0}\right)\left(A_{1}\right)\right] & = \\
& =(-1)^{n+1}\left[\left(\operatorname{ad}^{n-1} A_{0}\right)\left(A_{1}\right),\left(\operatorname{ad}^{n+2} A_{0}\right)\left(A_{1}\right)\right] .
\end{aligned}
$$

Applying ad $A_{0}$ to this equality, we obtain

$$
\begin{aligned}
& {\left[A_{0}, I\right]=(-1)^{n}\left[\left(\operatorname{ad}^{n} A_{0}\right)\left(A_{1}\right),\left(\operatorname{ad}^{n+2} A_{0}\right)\left(A_{1}\right)\right]=} \\
& =(-1)^{n+1}\left(\left[\left(\operatorname{ad}^{n} A_{0}\right)\left(A_{1}\right),\left(\operatorname{ad}^{n+2} A_{0}\right)\left(A_{1}\right)\right]+\right. \\
& \left.+\left[\left(\operatorname{ad}^{n-1} A_{0}\right)\left(A_{1}\right),\left(\operatorname{ad}^{n+3} A_{0}\right)\left(A_{1}\right)\right]\right) .
\end{aligned}
$$

On the other hand, applying ad $B_{0}$ to $\left[\left(\operatorname{ad}^{n-1} A_{0}\right)\left(A_{1}\right),\left(\operatorname{ad}^{n} A_{0}\right)\left(A_{1}\right)\right]=0$ and taking into account (174) and (171), one gets

$$
\left[\left(\operatorname{ad}^{n+2} A_{0}\right)\left(A_{1}\right),\left(\operatorname{ad}^{n} A_{0}\right)\left(A_{1}\right)\right]+\left[\left(\operatorname{ad}^{n-1} A_{0}\right)\left(A_{1}\right),\left(\operatorname{ad}^{n+3} A_{0}\right)\left(A_{1}\right)\right]=0 .
$$

Combining this with (185), we obtain $\left[A_{0}, I\right]=0$, which proves relation (183).

By Lemma 16] relation (177) implies that $\mathfrak{N}_{i+1} / \mathfrak{N}_{i}$ is commutative. Relation (176) says that in the quotient algebra $\mathfrak{N} / \cup_{i} \mathfrak{N}_{i}$ we have

$$
\left[\left(\operatorname{ad}^{k} A_{0}\right)\left(A_{1}\right),\left(\operatorname{ad}^{l} A_{0}\right)\left(A_{1}\right)\right]=0 \quad \forall k, l \in \mathbb{Z}_{+},
$$

which implies that this quotient of $\mathfrak{N}$ is solvable.

Theorem 26. The algebra $\mathfrak{N}$ is not quasi-finite.

Proof. In the quotient algebra $\mathfrak{N} / \cup_{i} \mathfrak{N}_{i}$ denote $c_{k}=\left(\operatorname{ad}^{k} A_{0}\right)\left(A_{1}\right)$. Consider the subalgebra $\mathfrak{g}$ of $\mathfrak{N} / \cup_{i} \mathfrak{N}_{i}$ generated by $B_{0}$ and $c_{k}$. Obviously, for a quasi-finite algebra any quotient algebra and any subalgebra of finite codimension are also quasi-finite. Therefore, it is sufficient to prove that the algebra $\mathfrak{g}$ is not quasi-finite.

Relations (186) say that $\left[c_{k}, c_{l}\right]=0$, while relations (174) and (171) imply $\left[B_{0}, c_{k}\right]=c_{k+3}$. Let $m_{k}, k \in \mathbb{Z}_{+}$, be a sequence of nonzero complex numbers satisfying $m_{k+3}=-(k+1) m_{k}$. Consider the following transitive action of $\mathfrak{g}$ on the manifold $M=\left\{(x, y) \in \mathbb{C}^{2} \mid x \neq 0, y \neq 0\right\}$

$$
c_{k} \mapsto \frac{m_{k}}{x^{k+1}} \frac{\partial}{\partial y}, \quad B_{0} \mapsto \frac{1}{x^{2}} \frac{\partial}{\partial x} .
$$

By Theorem 7 since the image of $\mathfrak{g}$ in $D(M)$ is infinite-dimensional, the algebra $\mathfrak{g}$ is not quasi-finite.

\section{NON-EXISTENCE RESULTS FOR BÄCKLUND TRANSFORMATIONS}

Theorem 27. Equation (169) is not connected by any Bäcklund transformation neither with the KdV equation nor with the nonsingular Krichever-Novikov equation.

Proof. Below a Lie subalgebra denoted by $\mathfrak{h}, \mathfrak{h}^{1}$, or $\mathfrak{h}^{2}$ is always supposed to be of finite codimension. The following lemma is obvious. 
Lemma 18. Let $\mathfrak{g}$ be a finite-dimensional semisimple Lie algebra. Suppose that a Lie algebra $\mathfrak{g}_{1}$ is obtained from a Lie algebra $\mathfrak{g}_{2}$ applying several times the operation of one-dimensional central extension. Then each of the following properties holds for $i=1$ if and only if it holds for $i=2$.

- There are a subalgebra $\mathfrak{h} \subset \mathfrak{g}_{i}$ and an epimorphism $\mathfrak{h} \rightarrow \mathfrak{g}$.

- For any subalgebra $\mathfrak{h} \subset \mathfrak{g}_{i}$ there is an epimorphism $\mathfrak{h} \rightarrow \mathfrak{g}$.

Set $\mathfrak{g}=\mathfrak{s l}_{2}(\mathbb{C})$. Let us prove first that there is no Bäcklund transformation between equation (169) and the nonsingular Krichever-Novikov equation. Combining Lemma 18 with Theorems 22, 24] and 14 we see that it is sufficient to prove that for any subalgebras $\mathfrak{h}^{1} \subset \mathfrak{R}, \mathfrak{h}^{2} \subset \mathfrak{N}$ there is an epimorphism $\mathfrak{h}^{1} \rightarrow \mathfrak{g}$, but there is no epimorphism $\mathfrak{h}^{2} \rightarrow \mathfrak{g}$.

There is a natural family of epimorphisms $\mathfrak{R} \rightarrow \mathfrak{g}$ parameterized by the points of the affine curve in $\mathbb{C}^{3}$ given by polynomials (161). Namely, for a point $\left(a_{1}, a_{2}, a_{3}\right)$ of the curve the generator $x_{i} \otimes \bar{v}_{i}$ of $\mathfrak{R}$ is mapped to $a_{i} x_{i} \in \mathfrak{g}$. Since $\mathfrak{h}^{1}$ is of finite codimension in $\mathfrak{R}$, there are polynomials $f_{i}\left(\bar{v}_{1}, \bar{v}_{2}, \bar{v}_{3}\right)$ and a point $\left(a_{1}, a_{2}, a_{3}\right)$ of the curve such that $x_{i} \otimes f_{i}$ belongs to $\mathfrak{h}^{1}$ and $f_{i}\left(a_{1}, a_{2}, a_{3}\right) \neq 0$ for all $i=1,2,3$. Then the restriction to $\mathfrak{h}^{1}$ of the corresponding homomorphism $\rho: \mathfrak{R} \rightarrow \mathfrak{g}$ is surjective, since the elements

$$
\rho\left(x_{i} \otimes f_{i}\right)=f_{i}\left(a_{1}, a_{2}, a_{3}\right) x_{i}, \quad i=1,2,3,
$$

span $\mathfrak{g}$.

Non-existence of an epimorphism $\mathfrak{h}^{2} \rightarrow \mathfrak{g}$ follows from Theorem [25] Indeed, suppose that there is an epimorphism $\rho: \mathfrak{h}^{2} \rightarrow \mathfrak{g}$. Since $\mathfrak{h}^{2} \cap \mathfrak{N}_{i}$ is solvable, we have $\rho\left(\mathfrak{h}^{2} \cap \mathfrak{N}_{i}\right)=0$ for all $i$. Therefore, there is an epimorphism

$$
\mathfrak{h}^{2} /\left(\mathfrak{h}^{2} \cap\left(\cup_{i} \mathfrak{N}_{i}\right)\right) \rightarrow \mathfrak{g},
$$

which is impossible, since $\mathfrak{N} / \cup_{i} \mathfrak{N}_{i}$ is solvable.

Let us now prove that there is no Bäcklund transformation between equation (169) and the KdV equation. Since, according to Theorem 17 each fundamental algebra of the $\mathrm{KdV}$ equation is obtained from $\mathfrak{g} \otimes \mathbb{C}[\lambda]$ applying several times the operation of one-dimensional central extension, it is sufficient to prove that for any subalgebra $\mathfrak{h}^{1} \subset \mathfrak{g} \otimes \mathbb{C}[\lambda]$ there is an epimorphism $\mathfrak{h}^{1} \rightarrow \mathfrak{g}$. Consider the natural family of epimorphisms

$$
\rho_{a}: \mathfrak{g} \otimes \mathbb{C}[\lambda] \rightarrow \mathfrak{g}, \quad g \otimes f(\lambda) \mapsto f(a) g, \quad a \in \mathbb{C} .
$$

Since $\mathfrak{h}^{1}$ is of finite codimension, for some of these epimorphisms its restriction to $\mathfrak{h}^{1}$ is surjective.

\section{ACKNOWLEDGEMENTS}

The author is deeply grateful to I. S. Krasilshchik for careful reading of the paper and many valuable comments. The author thanks also R. Martini and V. V. Sokolov for useful discussions.

\section{REFERENCES}

[1] A. V. Bocharov, V. N. Chetverikov, S. V. Duzhin, N. G. Khor'kova, I. S. Krasil'shchik, A. V. Samokhin, Yu. N. Torkhov, A. M. Verbovetsky, and A. M. Vinogradov. Symmetries and Conservation Laws for Differential Equations of Mathematical Physics. Amer. Math. Soc., Providence, RI, 1999. 
[2] R. Dodd and A. Fordy. The prolongation structures of quasipolynomial flows. Proc. Roy. Soc. London Ser. A 385 (1983), 389-429.

[3] J. D. Finley and J. K. McIver. Prolongations to higher jets of Estabrook-Wahlquist coverings for PDEs. Acta Appl. Math. 32 (1993), 197-225.

[4] V. V. Gorbatsevich, A. L. Onishchik, and E. B. Vinberg. Foundations of Lie theory and Lie transformation groups. Springer-Verlag, Berlin, 1997.

[5] S. Igonin. Horizontal cohomology with coefficients and nonlinear zero-curvature representations. Russian Math. Surveys 58 (2003), 180-182

[6] S. Igonin and R. Martini. Prolongation structure of the Krichever-Novikov equation. J. Phys. A: Math. Gen. 35 (2002), 9801-9810; arxiv.org/nlin.SI/0208006

[7] E. G. Kirnasov. On Wahlquist-Estabrook type coverings over the heat equation. Math. Notes 42 (1987), 732-739.

[8] I. S. Krasilshchik and A. M. Vinogradov. Nonlocal trends in the geometry of differential equations. Acta Appl. Math. 15 (1989), 161-209.

[9] I. S. Krasil'shchik, V. V. Lychagin, A. M. Vinogradov. Geometry of jet spaces and nonlinear partial differential equations. Gordon \& Breach, New York, 1986.

[10] J. Krasil'shchik and A. Verbovetsky. Homological Methods in Equations of Mathematical Physics. arxiv.org/math.DG/9808130

[11] I. M. Krichever and S. P. Novikov. Holomorphic bundles over algebraic curves, and nonlinear equations. Russian Math. Surveys 35 (1980), 53-80.

[12] M. Marvan. On zero-curvature representations of partial differential equations. Differential geometry and its applications (Opava, 1992), 103-122. Math. Publ., 1, Silesian Univ. Opava, 1993.

[13] T. Nagano. Linear differential systems with singularities and an application to transitive Lie algebras. J. Math. Soc. Japan 18 (1966), 398-404.

[14] G. H. M. Roelofs and R. Martini. Prolongation structure of the Landau-Lifshitz equation. J. Math. Phys. 34 (1993), 2394-2399.

[15] C. Rogers and W. K. Schief. Bäcklund and Darboux transformations. Cambridge Univ. Press, Cambridge, 2002

[16] C. Rogers and W. F. Shadwick. Bäcklund transformations and their applications. Academic Press, New York, 1982.

[17] V. V. Sokolov. On the hamiltonian property of the Krichever-Novikov equation. Soviet. Math. Dokl. 30 (1984), 44-46.

[18] S. I. Svinolupov, V. V. Sokolov, and R. I. Yamilov. Bäcklund transformations for integrable evolution equations. Soviet Math. Dokl. 28 (1983), 165-168.

[19] H. N. van Eck. The explicit form of the Lie algebra of Wahlquist and Estabrook. A presentation problem. Nederl. Akad. Wetensch. Indag. Math. 45 (1983), 149-164.

[20] H. N. van Eck. A non-Archimedean approach to prolongation theory. Lett. Math. Phys. 12 (1986), 231-239.

[21] A. Verbovetsky. Notes on the horizontal cohomology. Secondary calculus and cohomological physics (Moscow, 1997), 211-231. Contemp. Math., 219, Amer. Math. Soc., Providence, RI, 1998.

[22] H. D. Wahlquist and F. B. Estabrook. Prolongation structures of nonlinear evolution equations. J. Math. Phys. 16 (1975), 1-7.

Department of Mathematics, Utrecht University, P.O. Box 80010, 3508 TA Utrecht, THE NETHERLANDS

E-mail address: igonin@mccme.ru 